\title{
Identifying Agent's Information Sets: an Application to a Lifecycle Model of Schooling, Consumption and Labor Supply*
}

\author{
Salvador Navarro ${ }^{\dagger}$ and Jin Zhou ${ }^{\ddagger}$
}

January 19, 2017

\begin{abstract}
We adapt the insight of Cunha, Heckman, and Navarro (2005) to develop a methodology that distinguishes information unknown to the econometrician but forecastable by the agent from information unknown to both, at each point in an agent's lifecycle. Predictable variability and uncertainty have different implications in terms of welfare, especially when markets are incomplete. We apply our procedure in the context of an incomplete markets lifecycle model of consumption, labor supply, and schooling decisions, when borrowing limits arise from repayment constraints. Using microdata on earnings, hours worked, schooling choices, and consumption of white males in the US, we infer the agent's information set. We then estimate the model using the identified agent's information set. We find that $52 \%$ and $56 \%$ of the variance of college and high school log wages respectively are predictable by the agent at the time schooling choices are made. When we complete the market, college attendance increases from $48 \%$ to $59 \%$, about half of this increase is due to uncertainty, and the other half because of the borrowing limits. To illustrate the importance of assumptions about what is forecastable by the agent, we simulate a minimum wage insurance policy under different assumptions about the information available to the agents in the model. When we allow for asymmetric information between the insurance institution and the individual, adverse selection turns profits negative. Consumer welfare, however, increases by about $28 \%$ when we give individuals access to their estimated information set regardless of asymmetries.
\end{abstract}

${ }^{*}$ We would like to thank James Heckman, Flavio Cunha, Lance Lochner, Lars Hansen, Paul Schrimpf, Robert Townsend, Alessandro Barbarino, Pedro Carneiro, Victor Chernozhukov, Jeremy Fox, Bruce Hansen, Kenneth Judd, John Kennan, Rasmus Lentz, Rosa Matzkin, Ronni Pavan, David Rivers, Alejandro Rodriguez, Ananth Seshadri, and an anonymous referee for their helpful comments. Navarro acknowledges support from the Canadian Social Science and Humanities Research Council, Grant No. R4895A04. Zhou acknowledges support from the Center for the Economics of Human Development's Asian Family in Transition Initiative.

${ }^{\dagger}$ Department of Economics, University of Western Ontario. E-mail: s.navarro.lozano@gmail.com

${ }_{\ddagger}^{\ddagger}$ Center for the Economics of Human Development, University of Chicago. E-mail: jinzhou@uchicago.edu 


\section{Introduction}

The use of empirically implementable stochastic dynamic models to understand agents' behavior is common practice in the Economics literature. These models have been the workhorse of Macroeconomics research for a long time, and are becoming more and more common in Labor Economics (see Keane et al., 2011; Rogerson and Shimer, 2011 for examples and reviews). A common feature of these models is that the analyst needs to specify the information structure of the problem, in particular what is known to the agent making decisions at each point in time. ${ }^{1}$ In this paper we adapt the insight of Carneiro et al. (2003) and Cunha et al. (2005) to develop and implement a methodology that distinguishes information unknown to the econometrician but forecastable by the agent, and information unknown to both at each period. The key to measuring uncertainty using our procedure is to notice that individual choices reflect all the information known to the individual at a given time. ${ }^{2}$ From the perspective of the agent, differences between expected outcomes and actual realizations of these outcomes (i.e., innovations) should not help explain current choices (see Sims, 1972). If these "innovations" explain current choices it means that they are not truly innovations, so the expectations we as analysts are assigning to the agent, i.e., the information set we have assumed, is incorrect.

The procedure we propose is semiparametric in nature. It can be used independently of a particular model specification, as long as one considers families of models with the same determinants of choice. In this paper, however, we develop our procedure in the context of a (semiparametrically identified) structural model of schooling choice, labor supply, and consumption allocation under uncertainty, in which borrowing constraints arise from repayment constraints. ${ }^{3}$ To implement our procedure, we use an independent factor model to analyze wage dynamics.

In the context of the model we propose, being able to separate what is really ex-ante uncertainty from the perspective of the agent making choices is needed to avoid problems with model misspecification related to expectations. It is also crucial as the welfare consequences of uncertainty are very different from those of predictable (by the agent) variability. Furthermore, as pointed out by Lochner and MongeNaranjo (2016), information on ex-ante risks facing the individual are a crucial input into the design of optimal policies like student loan programs. It is also an ingredient for exercises that seek to quantify the degree of insurance available to agents (Blundell et al., 2008; Kaufmann and Pistaferri, 2009).

Our paper also contributes to to the literature on schooling choice by explicitly looking at the role played by uncertainty as a determinant of schooling. The idea is closely related to the work of Carneiro et al. (2003) and Cunha et al. (2005) in which a similar methodology is applied to extract agent's information at the schooling date. Carneiro et al. (2003) assume no credit markets operate. Cunha et al. (2005) and Cunha and Heckman (2016) assume an economy with perfect credit markets. We investigate an econ-

\footnotetext{
${ }^{1}$ There is a literature that, instead, uses data on individuals' subjective expectations to estimate these models. See van der Klaauw (2012) for an example. The main concern with the use of expectations data is that they are usually measured at a point in time, not repeatedly. However, as the agent's information evolves over time, his expectations are likely to change as well. See Kaufmann and Pistaferri (2009) for a related idea that uses assumptions on the time series process of outcomes to sidestep this difficulty.

${ }^{2}$ A similar idea motivates the work on the permanent income hypothesis of Flavin (1981) and Pistaferri (2001).

${ }^{3}$ See Huggett (1993), Aiyagari (1994) and Gourinchas and Parker (2002).
} 
omy in which some credit markets operate, and borrowing limits arise from repayment constraints. ${ }^{4}$ In terms of model setup, our paper is closest to Hai and Heckman (2016). ${ }^{5}$ The focus of our paper, however, is different from theirs. We focus on the importance of identifying the uncertainty faced by the agent, even after conditioning on unobserved ability, as well as its evolution over time. ${ }^{6}$

We estimate the model using pooled data on white males from the NLSY79 and PSID. ${ }^{7}$ Our estimates allow us to study the relative importance of uncertainty as a determinant of college attendance, as well as other determinants that have been studied in the literature, like ability (e.g., Cawley et al., 2000; Taber, 2001; Belzil and Hansen, 2002), and borrowing constraints (e.g., Kane, 1996; Carneiro and Heckman, 2002; Cameron and Taber, 2004; Brown et al., 2012; Lochner and Monge-Naranjo, 2011).

Our empirical results show that, at the time schooling decisions are made, about half of the unobserved (to the analyst) wage variation is predictable to the agent. In particular, the estimates of the model imply that $52 \%$ of the unexplained variance in college log wages is predictable by the agent at age 18 . This fraction is $56 \%$ for high school. This is similar to the results obtained by Cunha et al. (2005) and Storesletten et al. (2004), slightly smaller than Cunha and Heckman (2016), and much smaller than what Keane and Wolpin (1997) obtain. In fact, while the total unobserved variance of college log wages is higher than that of high school (i.e., the unobserved variance from the analyst's perspective), the variance of the uncertain components of wages becomes larger for college compared to high school under our estimated information set for the agent. We also find that, when people are allowed to smooth consumption by operating in a complete market setting, college attendance increases by roughly $12 \%$ points. We decompose this effect into a pure uncertainty effect, which accounts for about half of the increase, while the rest is due to borrowing constraints.

In order to illustrate the importance of assumptions regarding ex-ante uncertainty, we then simulate a policy in which agents can insure against adverse wage shocks. We simulate the effects of this minimum wage insurance policy under different assumptions about what is included in the information sets of the individuals and the institution offering the insurance (the "bank"). In particular, we show how one can potentially use our procedure to study asymmetries in the information available to the agent and to the bank, and the effects that adverse selection may have on college attendance and on bank profits. ${ }^{8}$ We find that assumptions about the information set matter a lot. In terms of the profits made by the institution

\footnotetext{
${ }^{4}$ See Huggett (1993), Aiyagari (1994), and Gourinchas and Parker (2002).

${ }^{5}$ Our specification of the borrowing limit is simpler than theirs as we don't include student loans, consumption floors or post-school endogenous human capital investments. Furthermore, their borrowing limit takes into consideration the individual rationality constraint of working and endogenous human capital accumulation. Also, the data they employ comes from NLSY97 while we pool data from NLSY79 and PSID.

${ }^{6}$ For example, both Keane and Wolpin (2001) and Cameron and Heckman (2001) estimate their dynamic models of schooling assuming that what is known by the agent and what is known by the econometrician at each point in time. While they allow for unobserved heterogeneity, it is essentially treated as an initial condition. Once the econometrician conditions on the initial heterogeneity, the evolution of the information set of the agent is given. However, there is no prior reason why one should assume that the evolution of what is unknown to the analyst and what is unknown to the agent coincide.

${ }^{7}$ We also contribute to the literature on the estimation of the Frisch elasticity of labor supply. By focusing on longer periods, we circumvent the need to distinguish between extensive and intensive margins that has been the focus of the recent literature (see Peterman, 2016). Of course, we have to make the stronger assumption that time within our longer period (6 years as opposed to the standard 1 year period) is "perfectly" substitutable. Our estimated Frisch elasticity of labor supply of 1 is in the middle of the estimates obtained from both the Macro and Micro literatures.

${ }^{8}$ See also Chatterjee and Ionescu (2012) for a related analysis on asymmetric information and loan forgiveness.
} 
offering the insurance, having access to the same information as the agent (under the "naive" assumptions that all the unobservables to the econometrician represent uncertainty to the agent) generates small positive profits. However, when we simulate what would happen if the agent has access to the information set we estimate for him, while the bank does not have access to this information, profits become negative. This happens because the information asymmetries give rise to adverse selection. Individuals who are more likely to have low wages in the future, are much more likely to buy the insurance, and the bank cannot price the insurance correctly. When we give the bank access to the same information as the agent, insurance premiums for the bad risks increase substantially, and profits become positive again. We also find that giving individuals access to the information set we estimate as the right one, increases welfare by about $28 \%$ relative to the case where uncertainty to the agent and to the econometrician coincide, regardless of what information the bank has access to. In terms of schooling, we find that agents who buy the minimum wage insurance have less incentives to go to college.

The rest of the paper proceeds as follows. Section 2 presents the version of the incomplete markets model of consumption, labor supply, and schooling decisions that we use in the rest of the paper. The methodology used to infer the elements of the agent's information set is explained in Section 3. In passing, we briefly sketch how semiparametric identification of the model is achieved. Appendix 1 presents a formal identification analysis. Section 4 describes the data we use and the parameterization of the model. In Section 5, we present our analysis of the agent's information set and estimates of the model using the appropriate information. The insurance policy simulation illustrating the importance of the informational assumption made by the analyst is in Section 6. Section 7 concludes.

\section{The Model}

\subsection{The Decision Process}

Individuals in our model live for $T+1$ periods and maximize expected lifetime utility in a world in which all risks arise from labor market risks and are idiosyncratic. The timing of the model works as follows. At the beginning of time $(t=0)$, an individual who just finished high school has to decide whether to attend college or simply stop schooling and enter the labor market. In order to make this decision, the agent considers his expectations about the wage sequence associated with each schooling level $s$. For each possible schooling choice, the agent selects an optimal intertemporal consumption allocation rule, and a rule for the proportion of time spent working. Given these optimal rules, the agent calculates the expected utility associated with choosing to stop at high school or move on to college. He then makes the schooling choice that maximizes his expected utility net of schooling costs.

Let $W_{i, s, t}$ denote the wage at time $t$ of an individual $i$ who has schooling level $s \in\{h s, c o l\}$ (hs=high school, col=college), $n_{i, t} \in[0,1]$ the fraction of the time the individual spends working, $H_{i, t}=\bar{H}_{s, t} n_{i, t}$ total hours worked (out of a max of $\bar{H}_{s, t}$ possible hours), ${ }^{9} Y_{i, s, t}=W_{i, s, t} H_{i, t}$ the earnings of the individual

\footnotetext{
${ }^{9}$ We assume that individuals always work positive hours, but see our discussion of the data. We also assume that, while in college, individuals can only work half time. That is, we assume that $\bar{H}_{s, t}=\bar{H}$ except when $s=c o l$ and $t=1$ (i.e., while in college) where $\bar{H}_{c o l, 1}=\frac{\bar{H}}{2}$.
} 
$i$, and $\rho$ the discount rate. Agents can borrow and lend subject to a repayment constraint at the end of life using a non-state-contingent asset, $A_{i, t}$, which pays an (assumed) riskless and constant return at a rate $r$.

The individual derives utility from consumption and work via a utility function that we denote by $u\left(C_{i, t}, H_{i, t}\right)$. Since we do not model retirement explicitly, we assume that the terminal period utility function is given by some function, $\widetilde{u}\left(Y_{i, s, T+1}^{M I N}, A_{i, T}\right)$. Utility at retirement depends on the amount of assets the agent has accumulated, as well as on some guaranteed income during retirement, $Y_{i, s, T+1}^{M I N}$, which we generically call "social security income".

Finally, we let $\mathcal{I}_{i, t}$ denote the state variables of the problem at time $t$. We call these state variables the information set of the agent, as they summarize the information that is available (and used) by the agent at time $t$. As we impose more structure on the problem, we will be more precise about what is in this information set. For now, it is enough to note that it includes all the past and current realizations of wages, the interest rate $r$, and the asset stock. The agent may also have some information about future wages. Determining whether this is the case, and trying to quantify the importance of this information are the main contributions of this paper.

More precisely, given schooling level $s$, the agent's problem at period $t>0$ is to select how much of his time to spend working, and, given available resources, how much to consume (which determines how much to transfer to the next period). The value function given information set $\mathcal{I}_{i, t}$ is thus given by

$$
\mathscr{V}_{i, s, t}\left(\mathcal{I}_{i, t}\right)=\max _{H_{i, t}, A_{i, t}} u\left(C_{i, t}, H_{i, t}\right)+\frac{1}{1+\rho} E\left(\mathscr{V}_{i, s, t+1}\left(\mathcal{I}_{i, t+1}\right) \mid \mathcal{I}_{i, t}\right)
$$

If we let $\mathbb{1}(t=1, s=c o l)$ be an indicator function that takes value one if the individual is in college, and $D_{i}$ be the direct cost of schooling (tuition) for individual $i$, the budget constraint implies that

$$
C_{i, t}=Y_{i, s, t}+(1+r) A_{i, t-1}-A_{i, t}-\mathbb{1}(t=1, s=\operatorname{col}) D_{i}
$$

Furthermore, we assume that agents cannot enter the terminal period in debt, which imposes a constraint (sometimes called the natural borrowing limit) on how much the individual can borrow at every period: ${ }^{10}$

$$
A_{i, t} \geq-Y_{i, s, T+1}^{M I N} /(1+r)^{T+1-t}
$$

where $Y_{i, s, T+1}^{M I N}$ is guaranteed income at retirement that we call "social security". In general, equation 3 would be the present value of the minimum possible income the individual can have at $t$. Since we do not impose any restrictions on the support of wages, it is always possible for an agent to draw a wage of zero. As a consequence, the minimum value of income he may have at $t$ is zero and no other terms enter equation $3 .^{11}$

\footnotetext{
${ }^{10}$ See Huggett (1993), Aiyagari (1994), Gourinchas and Parker (2002).

${ }^{11}$ Hai and Heckman (2016) have a similar model setup to ours, where a natural borrowing limit also arises as a consequence of repayment constraints. However, since they allow individuals to have zero labor supply with positive income and endogenous human capital investment, their borrowing limit is endogenous as it must satisfy the individual rationality constraint of working. See also Zhang (1997) for a similar idea on how to generate endogenous borrowing limits because of participation constraints in Aiyagari style models.
} 
If the utility function satisfies standard conditions (i.e., concavity and $\lim _{C \rightarrow 0} \partial u(C, H) / \partial C=\infty$ ) the limit imposed by equation 3 will not be binding. It will, however, still affect the individual's optimal choices. For this reason, and for concreteness, we still refer to it as a "borrowing limit".

From the agent's perspective (i.e., given his information at time $t$ ), the solution to the maximization problem in equation (1) consists of a trio of time-schooling indexed functions: a policy function that tells him how much to work this period

$$
H_{i, t}^{*}=\mathscr{H}_{s, t}\left(\mathcal{I}_{i, t}\right)
$$

a policy function that tells him how much to consume this period

$$
C_{i, t}^{*}=\mathscr{C}_{s, t}\left(\mathcal{I}_{i, t}\right)
$$

and the value function

$$
V_{i, t}^{*}=\mathscr{V}_{s, t}\left(\mathcal{I}_{i, t}\right)
$$

that gives the utility of working $H_{i, t}^{*}$ and consuming $C_{i, t}^{*}$ this period, and then following his optimal rules for $\tau>t$.

Once the agent solves the labor and consumption allocation problems, he can get the value associated with each $s$ at time $t=1$. At period $t=0$, the agent selects the schooling level $s$ that maximizes his expected utility net of "psychic" costs, $P .{ }^{12}$ That is, he will attend college if

$$
E\left(\mathscr{V}_{c o l, 1}\left(\mathcal{I}_{i, 1}\right)-\mathscr{V}_{h s, 1}\left(\mathcal{I}_{i, 1}\right)-P_{i} \mid \mathcal{I}_{i, 0}\right)>0
$$

\subsection{Specification of the Model}

Wages for individual $i$ at time $t$ at schooling level $s$ are written as

$$
\ln W_{i, s, t}=\mu_{s, t}\left(X_{i, t}\right)+U_{i, s, t}
$$

where $X_{i, t}$ represents variables that the econometrician observes and $U_{i, s, t}$ variables not observed by the econometrician. We assume that the agent knows all of the variables in $X$ at all times, and that $U_{i, s, t}$ is revealed to him at period $t$. He may also know all or part of each $\left(U_{i, s, \tau}, \tau=t+1, \ldots, T\right)$ at time $t$. Uncertainty is thus associated with $\left\{U_{i, s, \tau}\right\}_{\tau=t+1}^{T}$.

We write psychic costs in the schooling choice equation (7) as a function of variables $Z$ that are observed by both the analyst and the agent. $\zeta$ represents variables not observed by the econometrician which may be known to the agent at $t=0$. The psychic cost of schooling is

$$
P_{i}=\phi\left(Z_{i}\right)+\zeta_{i}
$$

\footnotetext{
${ }^{12}$ We refer to the schooling choice period as $t=0$ as a convenience. It is meant to be taken as a shorthand for allowing the agent to make their schooling choice right before period 1 starts. It is a simple way to allow for the information set used by the agent at schooling choice time to differ from the one used when consumption choices are made.
} 
The utility function is of the CRRA form

$$
u(C, H)=\frac{C^{1-\psi}-1}{1-\psi}-h \frac{n^{1+\frac{1}{\varphi}}}{1+\frac{1}{\varphi}},
$$

where $n=H / \bar{H}, \psi \geq 0$ is the coefficient of relative risk aversion, $h>0$ weights the utility of leisure, and $\varphi \geq 0$ is the Frisch elasticity of labor supply. We model utility in the terminal period as

$$
\widetilde{u}\left(Y_{i, s, T+1}^{M I N}, A_{i, T}\right)=b \frac{\left(Y_{i, s, T+1}^{M I N}+(1+r) A_{i, T}\right)^{1-\chi}}{1-\chi},
$$

where $b$ weights the utility in the terminal period. Since we do not model retirement explicitly, we let the function have a different relative risk aversion parameter $\chi$.

Finally, we assume that the data on both hours and consumption contain measurement errors. We observe noisy measures

$$
\begin{gathered}
\ln \widehat{H}_{i, t}=\ln H_{i, t}+\delta_{H}^{\prime} K_{i, t}^{H}+\xi_{i, t}^{H}, \\
\ln \widehat{C}_{i, t}=\ln C_{i, t}+\delta_{C}^{\prime} K_{i, t}^{C}+\xi_{i, t}^{C} .
\end{gathered}
$$

Here, $H_{i, t}, C_{i, t}$ are "true" hours and consumption, $\widehat{H}_{i, t}, \widehat{C}_{i, t}$ are measured hours and consumption in the data. We allow for measurement errors to depend on observables $K_{i, t}$, since we combine different datasets. For the case of consumption, it is a natural assumption in our context since consumption is measured at the household level, and then we impute it to the individual. ${ }^{13} K_{i, t}$ includes variables that control for the household structure as well as dataset of origin. $\xi_{i, t}$ is an unobserved term assumed to capture the rest.

\section{Inferring the Agent's Information Set}

The econometrician must know $\mathcal{I}_{i, t}$ in order to solve the model and develop estimating equations. Any conclusion extracted from the model relies crucially on the assumptions made about what constitutes uncertainty facing the agent. It is thus important to develop a procedure to allow the analyst to separate the components of the agent's information set from what is unknown to him. We now turn our attention to this topic, sketching identification of the model in the process. Appendix 1 provides a formal proof of identification. We begin by describing the assumptions we will make regarding the specification of the unobservables to the econometrician in the wage and psychic cost equations. In particular, we assume that the unobservables in wages and psychic costs can be factor analyzed. We then illustrate how we generalize the procedure to determine the agent's information set in Cunha et al. (2005). Appendix 2 contains a more general version of the procedure that can be implemented independently of the factor structure we impose below.

\footnotetext{
${ }^{13}$ Imputation is done by dividing total consumption over the square root of the total number of members in the household.
} 


\subsection{The Factor Structure and the Arrival of Information}

In order to separate unobserved (to the econometrician) variability from the uncertainty facing agents, it is useful to assume that the unobservables for agent $i$ can be factor analyzed in the following way:

$$
\begin{aligned}
U_{i, s, t} & =\theta_{i}^{\prime} \alpha_{s, t}+\varepsilon_{i, s, t} \\
\zeta_{i} & =\theta_{i}^{\prime} \lambda+\omega_{i} .
\end{aligned}
$$

$\theta_{i}$ is a vector of mean zero "factors", with $\theta_{i, j} \Perp \theta_{i, k}$ for all $j \neq k, j, k \in\{1, \ldots, T-1\}$ (i.e., the elements of $\theta_{i}$ are mutually independent). $\varepsilon_{i, s, t}$ and $\omega_{i}$ are also mean zero random variables called "uniquenesses". Uniquenesses, factors, and measurement errors, are all assumed mutually independent of each other for all schooling levels $s$ and time periods $t$. This assumption implies that, conditional on the observables, all the dependence in the model is captured by the factors, $\theta$.

The equations in (14) are only a statistical decomposition and, by themselves, are not informative about what is known to the agent at period $t$. They provide a useful device for extracting components of uncertainty from future outcomes. Let the $j^{\text {th }}$ element of the vector $\theta_{i}$ be denoted as $\theta_{i, j}$, with associated loadings given by $\alpha_{s, t, j}$. For identification purposes, we assume that the factor structure is such that wages in the first period are affected only by the first element of $\theta_{i}$ (i.e., $\theta_{i, 1}$ ), so the loadings $\alpha_{s, t, j}$ for $j>1$ and $t \geq 1$ are all zero. Earnings in period 2 are affected by the first two elements of $\theta_{i}$ (i.e., $\theta_{i, 1}, \theta_{i, 2}$ ), the next period by the first three, and so on up to period $T-1$. As will become clearer below, identifying the model will require us to not have a new factor in the last period. The matrix of loadings for wages given schooling level $s$ is thus given by

Table 1

\begin{tabular}{|c|c|c|c|c|c|c|c|}
\hline \multirow{2}{*}{ Time } & \multicolumn{7}{|c|}{ Loadings for factor } \\
\cline { 2 - 8 } & $\theta_{1}$ & $\theta_{2}$ & $\theta_{3}$ & $\ldots$ & $\theta_{T-3}$ & $\theta_{T-2}$ & $\theta_{T-1}$ \\
\hline 1 & $\alpha_{s, 1,1}$ & 0 & 0 & $\ldots$ & 0 & 0 & 0 \\
\hline 2 & $\alpha_{s, 2,1}$ & $\alpha_{s, 2,2}$ & 0 & $\ldots$ & 0 & 0 & 0 \\
\hline 3 & $\alpha_{s, 3,1}$ & $\alpha_{s, 3,2}$ & $\alpha_{s, 3,3}$ & $\ldots$ & 0 & 0 & 0 \\
\hline$\ldots$ & $\ldots$ & $\ldots$ & $\ldots$ & $\ldots$ & $\ldots$ & $\ldots$ & $\ldots$ \\
\hline$T-2$ & $\alpha_{s, T-2,1}$ & $\alpha_{s, T-2,2}$ & $\alpha_{s, T-2,3}$ & $\ldots$ & $\alpha_{s, T-2, T-3}$ & $\alpha_{s, T-2, T-2}$ & 0 \\
\hline$T-1$ & $\alpha_{s, T-1,1}$ & $\alpha_{s, T-1,2}$ & $\alpha_{s, T-1,3}$ & $\ldots$ & $\alpha_{s, T-1, T-3}$ & $\alpha_{s, T-1, T-2}$ & $\alpha_{s, T-1, T-1}$ \\
\hline$T$ & $\alpha_{s, T, 1}$ & $\alpha_{s, T, 2}$ & $\alpha_{s, T, 3}$ & $\ldots$ & $\alpha_{s, T, T-3}$ & $\alpha_{s, T, T-2}$ & $\alpha_{s, T, T-1}$ \\
\hline
\end{tabular}

We assume that elements of $\theta_{i}$ are revealed to the agent through their effects on wages, i.e., at $t=1$ the agent learns $\theta_{i, 1}$, at $t=2$ he learns $\theta_{i, 2}$ and so on. ${ }^{14}$ However, the agent might be able to forecast future elements of $\theta_{i}$ that affect future wages but not past and currently observed wages. Let $\theta_{i}(t)=\left(\theta_{i, 1}, \ldots, \theta_{i, t}\right)$ denote those elements of $\theta_{i}$ that affect wages at or before $t$, and let $\bar{\theta}_{i}(t)=\left(\theta_{i, t+1}, \ldots, \theta_{i, T-1}\right)$ denote those elements of $\theta_{i}$ that affect wages after $t$. We further separate $\bar{\theta}_{i}(t)$ into two components, $\left(\bar{\theta}_{i}^{k}(t), \bar{\theta}_{i}^{u}(t)\right)$ where $\bar{\theta}_{i}^{k}(t)$ is known by the agent at time $t$ and in $\mathcal{I}_{i, t}$, while $\bar{\theta}_{i}^{u}(t)$ is unknown by the agent at time $t$ and is not in $\mathcal{I}_{i, t}$. The empirical challenge is to determine which future wage factors are known (i.e., $\bar{\theta}_{i}^{k}(t)$ )

\footnotetext{
${ }^{14}$ This is an assumption, since only observing wages involves a signal extraction problem to determine the factors given $\varepsilon_{i, s, t}$. See assumption I-2 below.
} 
and which are unknown (i.e., $\left.\bar{\theta}_{i}^{u}(t)\right)$ by the agent at each date $t$.

The following assumptions are made about the arrival of information

(I-1) The information revelation process of the agent is such that he either knows element $j$ of $\theta_{i}, \theta_{i, j}$ $(j=1, \ldots T-1)$, or he only knows its marginal distribution. Revelation of information when it happens, is instantaneous and permanent (i.e., the agent does not "forget" factors they have learned in the past).

(I-2) At period $t$, the agent knows $\left\{\varepsilon_{i, s, \tau}\right\}_{\tau=1}^{t}$ and the elements of $\theta_{i}(t)$, that is those elements of $\theta_{i}$ that affect wages in that period (or in any previous periods). If $\theta_{i, \tau}$ affects wages at $\tau \leq t$, then it is known by the agent at time $t$.

(I-3) Agents have rational expectations so that the expectations they take and the mathematical expectation operator with respect to the actual distributions in the data coincide. In particular, they understand the structure of the arrival of information. That is, they know in advance when and which elements of $\bar{\theta}_{i}^{u}(t)$ they will learn, even if they do not know the particular values they will take.

Assumption I-1 allows for the fact that some future wage factors maybe learned beforehand by the agent, and the extent of this learning evolves over time. This is what distinguishes our procedure from more standard procedures in which the unobservables to the econometrician are assumed to be the same, or that they coincide conditional on some time-invariant heterogeneity (e.g., a fixed effect). It does, however, rule out Bayesian learning models based on observing a signal (e.g., wages). The rest of the information structure of the model is assumed to be such that the agent has knowledge of the parameters of the model (e.g., $\rho, \psi, \mu(X)$ ) as well as knowledge of the observables $X_{i}, K_{i}, Z_{i}{ }^{15}$ and the uniqueness in the cost function $\omega_{i}$. The econometrician never observes $\theta_{i}$. By assumption, $\left\{\varepsilon_{i, s, \tau}\right\}_{\tau=t+1}^{T}$ is not part of the agent's information set $\mathcal{I}_{i, t}$.

\subsection{Identifying the Information Set Under the Factor Structure}

In this section we cast the problem of determining agent information sets in terms of the factor structure. The basic idea is that if element $j$ of $\bar{\theta}_{i}(t)$ belongs in the information set of the agent at time $t$, and the agent acts on it, ${ }^{16}$ it will affect the choices he makes at $t$. In particular it will affect the hours and consumption decisions. There is nothing special about the hours and consumption decisions. Any other decision variable that depends on future outcomes can also be used (e.g., schooling decisions).

We begin by noting that for any arbitrarily proposed (by the analyst) information set, $\widetilde{\mathcal{I}}_{i, t}$, it follows that $\ln \widehat{H}_{i, t}=\ln \mathscr{H}_{s, t}\left(\widetilde{\mathcal{I}}_{i, t}\right)+\delta_{H}^{\prime} K_{i, t}^{H}+\xi_{i, t}^{H}$, and similarly for consumption. That is, measured hours should equal those predicted by the model via the policy function, $\mathscr{H}_{s, t}$, plus measurement errors (i.e., $\left.\delta_{H}^{\prime} K_{i, t}^{H}+\xi_{i, t}^{H}\right)$. Notice, however, that this holds true for a whole class of models (i.e., entire families of policy functions) besides the ones arising from the particular model we proposed in Section 2. That hours and consumption will be a function of the state variables of the model (i.e., the information set) is independent of the particular form of the utility function or of the wage equations. In general, for a pair

\footnotetext{
${ }^{15}$ This assumption can be relaxed by modeling the stochastic process that generates these variables. For example, Keane and Wolpin (1997) have one $X$, experience in each sector, the accumulation of which they model.

${ }^{16}$ It can be the case that something is known to the agent at time $t$ but it does not affect his time $t$ choices. If these is the case, our procedure will have no power to detect these elements of the information set. That is, we can only detect elements of the information set known and acted on by the agent.
} 
of nonparametric functions $\left(\mathscr{G}_{s, t}^{H}, \mathscr{G}_{s, t}^{C}\right)$ of the proposed information set, we can write

$$
\begin{aligned}
\ln \widehat{H}_{i, t} & =\mathscr{G}_{s, t}^{H}\left(\widetilde{\mathcal{I}}_{i, t}\right)+\delta_{H}^{\prime} K_{i, t}^{H}+\xi_{i, t}^{H}, \\
\ln \widehat{C}_{i, t} & =\mathscr{G}_{s, t}^{C}\left(\widetilde{\mathcal{I}}_{i, t}\right)+\delta_{C}^{\prime} K_{i, t}^{C}+\xi_{i, t}^{C} .
\end{aligned}
$$

Notice the dynamic program does not need to be computed. ${ }^{17}$

The idea behind how to determine the information set of the agent is simple. Under the correct specification for the information set, anything else we add to the right hand side of equations (15) and (16) should have no predictive power. If we add something, and it has predictive power, it means that it should be part of the information set of the agent as it is a determinant of choices. Using this procedure based on $\left(\mathscr{G}_{s, t}^{H}, \mathscr{G}_{s, t}^{C}\right)$, the determination of the information set will still be valid for a general class of models predicting that hours and consumption can be written as in equations (15) and (16).

Given the factor structure and information assumptions (I-1) - (I-3), ${ }^{18}$ the only source of wage innovations "knowable" to the agent is given by $\bar{\theta}_{i}(t)$ (i.e., the factors that affect wages only in periods after $t$ ). Candidate information sets would then differ in terms of what elements of $\bar{\theta}_{i}(t)$ are assumed to be part of $\bar{\theta}_{i}^{k}(t)$ and $\bar{\theta}_{i}^{u}(t)$.

Implementation of the procedure is actually possible in one step, i.e., there is no need to try many candidate information sets. Start by assuming that the agents do not know the different elements of $\theta_{i}$ until they learn them when they start affecting wages. That is, the model (including the wage process) is estimated using the candidate information set $\widetilde{\mathcal{I}}_{i, t}$ that contains $X, Z$, assets, tuition, $\omega_{i},\left\{\varepsilon_{i, s, \tau}\right\}_{\tau=1}^{t}$, and $\theta_{i}(t)$, but contains no elements of $\bar{\theta}_{i}(t)$ before time $t$. Then, instead of basing the likelihood on equations (15) and (16), ${ }^{19}$ we use

$$
\begin{aligned}
& \ln \widehat{H}_{i, t}=\mathscr{G}_{s, t}^{H}\left(\widetilde{\mathcal{I}}_{i, t}\right)+\delta_{H}^{\prime} K_{i, t}^{H}+\xi_{i, t}^{H}+\bar{\theta}_{i}(t) \pi_{t}^{H} \\
& \ln \widehat{C}_{i, t}=\mathscr{G}_{s, t}^{C}\left(\widetilde{\mathcal{I}}_{i, t}\right)+\delta_{C}^{\prime} K_{i, t}^{C}+\xi_{i, t}^{C}+\bar{\theta}_{i}(t) \pi_{t}^{C}
\end{aligned}
$$

By assumption, the predicted hours and consumption $\left(\mathscr{G}_{s, t}^{H}\left(\widetilde{\mathcal{I}}_{i, t}\right), \mathscr{G}_{s, t}^{C}\left(\widetilde{\mathcal{I}}_{i, t}\right)\right)$ will not depend on $\bar{\theta}_{i}(t)$ since the agent integrates them out. The actual decisions, however, will be a function of the agent's true information set at $t$ which may contain elements of $\bar{\theta}_{i}(t)$. Hence, any non-zero elements of $\left(\pi_{t}^{H}, \pi_{t}^{C}\right)$ associated with coordinate $j$ of $\bar{\theta}_{i}(t)$ belong in the agent's information set at time $t$. That is, if the $j^{\text {th }}$ element of $\bar{\theta}_{i}(t)$ is actually part of the agent's information set, it will affect the hours or consumption decisions. As a consequence, the elements of $\pi_{t}^{C}$ associated with this $j^{\text {th }}$ element will be estimated to be different from zero. Since we assume that the agent's do not know $\bar{\theta}_{i}(t)$ at $t$, the first time element $j$ appears to affect choices is when it becomes known to the agent. For example, if $\theta_{i, 4}$ shows up as affecting consumption decisions at $t=2$ (i.e., its associated $\pi_{2}^{C}$ is estimated to be different from zero), we conclude that the agent knows factor 4 at time 2, even though it does not affect wages until 2 periods later.

\footnotetext{
${ }^{17}$ The idea that we do not necessarily need to solve the whole dynamic program to estimate some functions is not new. See Marschak (1953), and see Hotz and Miller (1993), Aguirregabiria and Mira (2002) and Carranza (2007) for recent examples.

${ }^{18}$ The assumption that the vector $\theta$ is independent of $X, Z$ is also imposed.

${ }^{19}$ Alternatively, they could be used to form moments to compare against the data in a GMM setting.
} 
The same idea can be applied to agent schooling choices. Estimating the schooling choice part of the model under $\widetilde{\mathcal{I}}_{i, 0}$ (with no elements of $\theta_{i}$ contained in it), and using ${ }^{20}$

$$
E\left(\mathscr{V}_{c o l, 1}\left(\tilde{\mathcal{I}}_{i, 1}\right)-\mathscr{V}_{h s, 1}\left(\tilde{\mathcal{I}}_{i, 1}\right)-P_{i} \mid \tilde{\mathcal{I}}_{i, 0}\right)+\theta_{i} \pi_{0}>0
$$

as the college choice rule, allows us to check for which elements of $\pi_{0}$ are different from zero.

\subsection{Identification of the Model}

Formal identification analysis of the factor model of equations (1) - (14) is established in Appendix 1. This section provides an intuitive sketch of the identification arguments used in the Appendix for the factor structure of log wages, as well as establishing identification of the preference parameters. ${ }^{21}$ In this paper, identification theory is used to understand what in principle can be recovered nonparametrically from the data. We then use flexible parametric forms to obtain estimates of our high dimensional econometric model.

Wages are identified by generalizing the arguments in Carneiro et al. (2003), Bonhomme and Robin (2010), and Cooley Fruehwirth et al. (2016). We assume that the problem of selection (i.e., that we only observe college wages for college graduates, and high school wages for high school graduates) is solved using the arguments in Appendix 1 which involve using variation in the $Z_{i}$ to achieve limit sets. We further assume that $X, Z, K \Perp \theta, \varepsilon_{h s}, \xi^{H}, \xi^{C}$, i.e., that the covariates are exogenous. Without loss of generality, we work with the system of log-wage equations for high school: $\ln W_{i, h s, t}=\mu_{h s, t}\left(X_{i, h s, t}\right)+\theta_{i} \alpha_{h s, t}+\varepsilon_{i, h s, t}, t \geq$ 1.

First, notice that any factor $\left\{\theta_{i, j}\right\}_{j=1}^{T-1}$ has no natural scale or sign. We set both by normalizing one loading for each element $\theta_{i, j}, j=1, \ldots, T-1$.. In particular, we normalize the loading for $\theta_{i, 1}$ in the first period, so that $\alpha_{h s, 1,1}=1$; we normalize the loading on $\theta_{i, 2}$ in the second period, so $\alpha_{h s, 2,2}=1$, and so on for the $T-1$ factors. For simplicity of exposition, we assume that $\left\{E \theta_{i, j}^{3} \neq 0\right\}_{j=1}^{T-1}$ in this section. This non-symmetry assumption is not necessary. In Appendix 1, we show how one can identify the model even if this is not the case.

We begin by forming

$$
\frac{E\left(U_{h s, 1} U_{h s, 2}^{2}\right)}{E\left(U_{h s, 1}^{2} U_{h s, 2}\right)}=\alpha_{h s, 2,1},
$$

so we can recover the loading for $\theta_{i, 1}$ in period 2 (remember that $\alpha_{h s, 1,1}=1$ by normalization). From $E\left(U_{h s, 1} U_{h s, 2}\right)=\alpha_{h s, 2,1} E \theta_{1}^{2}$, we can identify $E \theta_{1}^{2}$. Since $E\left(U_{h s, 1} U_{h s, t}\right)=\alpha_{h s, t, 1} E \theta_{1}^{2}, t>2$, we can identify all the loadings for $\theta_{i, 1}$. From $E\left(U_{h s, 1}^{2}\right)=E \theta_{1}^{2}+E \varepsilon_{h s, 1}^{2}$, we can recover $E \varepsilon_{h s, 1}^{2}$. $E\left(U_{h s, 1}^{2} U_{h s, 2}\right)=$ $\alpha_{h s, 2,1} E \theta_{1}^{3}$, allows us to identify $E \theta_{1}^{3}$. Finally, from $E\left(U_{h s, 1}^{3}\right)=E \theta_{1}^{3}+E \varepsilon_{h s, 1}^{3}$ we can recover $E \varepsilon_{h s, 1}^{3}$.

We next show how to identify all the loadings for $\theta_{i, 2}$. From

\footnotetext{
${ }^{20}$ Notice that, same as before, we can use a nonparametric function of $\widetilde{\mathcal{I}}_{i, 1}$ instead of the actual solution to the dynamic problem.

${ }^{21}$ Identification of the parameters of the "psychic" cost function is also established in Appendix 1. See also Heckman and Navarro (2007).
} 


$$
\frac{E\left(U_{h s, 2} U_{h s, 3}^{2}\right)-\alpha_{h s, 2,1} \alpha_{h s, 3,1}^{2} E \theta_{1}^{3}}{E\left(U_{h s, 2}^{2} U_{h s, 3}\right)-\alpha_{h s, 2,1}^{2} \alpha_{h s, 3,1} E \theta_{1}^{3}}=\alpha_{h s, 3,2}
$$

we recover the loading for factor 2 in period 3. From $E\left(U_{h s, 2} U_{h s, 3}\right)=\alpha_{h s, 2,1} \alpha_{h s, 3,1} E \theta_{1}^{2}+\alpha_{h s, 3,2} E \theta_{2}^{2}$, we can identify $E \theta_{2}^{2}$. Given the loadings of $\theta_{i, 1}, E \theta_{1}^{2}$, and $E \theta_{2}^{2}$, we can use

$$
\frac{E\left(U_{h s, 2} U_{h s, t}\right)-\alpha_{h s, 2,1} \alpha_{h s, t, 1} E \theta_{1}^{2}}{E \theta_{1}^{2}}=\alpha_{h s, t, 2}
$$

to recover $\alpha_{h s, t, 2}, t>3$. From $E\left(U_{h s, 2}^{2}\right)=\alpha_{h s, 1,2}^{2} E \theta_{1}^{2}+E \theta_{2}^{2}+E \varepsilon_{h s, 2}^{2}$, we recover $E \varepsilon_{h s, 2}^{2} . E\left(U_{h s, 2}^{2} U_{h s, 3}\right)=$ $\alpha_{h s, 2,1}^{2} \alpha_{h s, 3,1} E \theta_{1}^{3}+\alpha_{h s, 3,2} E \theta_{2}^{3}$, is enough to identify $E \theta_{2}^{3}$. Finally, from $E\left(U_{h s, 2}^{3}\right)=\alpha_{h s, 1,2}^{3} \theta_{1}^{3}+E \theta_{2}^{3}+E \varepsilon_{h s, 2}^{3}$ we can recover $E \varepsilon_{h s, 2}^{3}$.

To recover the remaining loadings, we proceed sequentially. By forming higher order cross moments we can recover $\left\{\left\{E \theta_{j}^{k}\right\}_{j=1}^{T-1},\left\{E \varepsilon_{h s, j}^{k}\right\}_{j=1}^{T}\right\}_{k=1}^{\infty}$. If these random variables satisfy conditions that allow their distributions to be characterized by their moments, the fact that we can recover all of the moments means that we can identify their marginal distributions nonparametrically. ${ }^{22}$

Given the normalizations we just made to the high school system of wages, making a similar set of normalizations to the college system would amount to setting the sign (and magnitude) of the unobserved covariance between college and high school log wages. To see this, notice that, given our assumption that the $\varepsilon_{i, s, t}$ are independent not only over time but also across $s$, the unobserved covariance of log wages in high school and college in period 1 is $\operatorname{cov}\left(U_{h s, 1}, U_{c o l, 1}\right)=\alpha_{c o l, 1} E \theta_{1}^{2}$. Clearly, setting $\alpha_{c o l, 1}=1$ would impose a strong restriction that the covariance is positive, and the magnitude would be fixed by the variance determined in the high school system. The same holds for other periods. As we show in Corollary 1 of Appendix 1, we do not need to impose any restrictions on the college factor loadings. The $\left\{\left\{\alpha_{c o l, t, j}\right\}_{j=1}^{T-1}\right\}_{t=1}^{T}$ can be recovered freely, and hence we do not restrict the sign or magnitude of the covariances between high school and college wages. ${ }^{23}$

Preferences, the discount factor, and measurement errors in hours and consumption can be identified from the usual first order condition and Euler equation arguments. From the first order condition for labor supply at an interior solution, $H_{i, t} \in\left(0, \bar{H}_{s, t}\right]$, we have

$$
C_{i, t}^{-\psi} W_{i, t} \bar{H}_{s, t}^{1+\frac{1}{\varphi}}=h H_{i, t}^{\frac{1}{\varphi}}
$$

\footnotetext{
${ }^{22}$ Loosely speaking, moments characterize a random variable if these moments do not grow "too" fast. See Theorem 30.1 in Billingsley (1995) for example. Of course, random variables are not necessarily characterized by their moments. In Appendix 1 in Theorem (3), we show that we can extend a theorem of Kotlarski (1967) to show nonparametric identification using characteristic functions in our setting.

${ }^{23}$ The fact that we can potentially identify this covariance is a direct result of our assumption of a common vector of factors affecting both high school and college wages. Without this restriction, this covariance is generally not identified. See, for example, the discussion of the generalized Roy model in French and Taber (2011).
} 
Replacing consumption and labor by their measured (with error) counterparts and taking logs we have

$$
\ln \widehat{H}_{i, t}=\varphi\left[\left(1+\frac{1}{\varphi}\right) \ln \bar{H}_{s, t}-\ln h\right]-\psi \varphi \ln \widehat{C}_{i, t}+\varphi \ln W_{i, t}+\delta_{H}^{\prime} K_{i, t}^{H}+\psi \varphi \delta_{C}^{\prime} K_{i, t}^{C}+\left(\xi_{i, t}^{H}+\psi \varphi \xi_{i, t}^{C}\right) .
$$

$\ln \widehat{C}_{i, t}$ in equation (19) is correlated with the residual via $\xi_{i, t}^{C}$. Under the assumption that measurement error is uncorrelated we can instrument for $\ln \widehat{C}_{i, t}$ with lagged $\ln \widehat{C}_{i, t-\tau}$ for $\tau>0$. Equation (19) thus identifies $\varphi, \psi, h$, the unique elements of $\delta_{H}$ and $\delta_{C},\left(\delta_{H}+\psi \varphi \delta_{C}\right)$ for the common elements of $K_{i, t}$, as well as the convolution $\xi_{i, t}^{H}+\psi \varphi \xi_{i, t}^{C}$.

In order to see how the remaining elements of the model are identified we use relatively standard Euler equation arguments. ${ }^{24}$ Using the first order condition of equation (6) and equations (10) and (13) it follows that

$$
E\left(\frac{1+r}{1+\rho}\left(\frac{\widehat{C}_{i, t+1} e^{\delta_{C}^{\prime} K_{i, t}^{C}+\xi_{i, t}^{C}}}{\widehat{C}_{i, t} e^{\delta_{C}^{\prime} K_{i, t+1}^{C}+\xi_{i, t+1}^{C}}}\right)^{-\psi}-1 \mid \mathcal{I}_{i, t}\right)=0 .
$$

Equation (20) is a standard consumption Euler equation, except that it contains measurement error and so the standard argument of Hansen and Singleton (1983) cannot be applied directly. ${ }^{25}$ Instead, we rewrite equation (20) as

$$
\frac{1+r}{1+\rho}\left(\frac{\widehat{C}_{i, t+1} e^{\delta_{C}^{\prime} K_{i, t}^{C}+\xi_{i, t}^{C}}}{\widehat{C}_{i, t} e^{\delta_{C}^{\prime} K_{i, t+1}^{C}+\xi_{i, t+1}^{C}}}\right)^{-\psi}=\eta_{i, t}+1
$$

where $\eta_{i, t}$ is expectational error which is a function of (among other things) the elements of $\theta_{i}$ not contained in $\mathcal{I}_{i, t}$. Taking logs and a linear approximation of $\eta_{i, t}+1$ around $\eta_{i, t}=0$ we obtain

$$
\ln \frac{\widehat{C}_{i, t+1}}{\widehat{C}_{i, t}}=\frac{1}{\psi} \ln \left(\frac{1+r}{1+\rho}\right)+\left(K_{i, t+1}^{C}-K_{i, t}^{C}\right)^{\prime} \delta_{C}+\left(\xi_{i, t+1}^{C}-\xi_{i, t}^{C}-\frac{\eta_{i, t}}{\psi}\right),
$$

From the fact that the interest rate $r$ is given and $\psi$ is identified above, it follows that we can identify the discount factor $\rho .^{26}$

We next proceed to look at the Euler equation in the terminal period

$$
-\left(\widehat{C}_{i, T} e^{-\delta_{C}^{\prime} K_{i, t}^{C}-\xi_{i, t}^{C}}\right)^{-\psi}+\frac{b(1+r)}{1+\rho}\left(Y_{i, s, T+1}^{M I N}+(1+r) A_{i, T}\right)^{-\chi}=0
$$

where there is no expectation with respect to $A_{T}$ since it is known at time $T$. For identification purposes it is helpful to rewrite equation (22) as

$$
\ln \widehat{C}_{i, T}=-\frac{1}{\psi} \ln \left(\frac{b(1+r)}{1+\rho}\right)+\frac{\chi}{\psi} \ln \left(Y_{i, s, T+1}^{M I N}+(1+r) A_{i, T}\right)-\delta_{C}^{\prime} K_{i, T}^{C}-\xi_{i, T}^{C} .
$$

\footnotetext{
${ }^{24}$ See Hansen and Singleton (1983), Browning and Lusardi (1996) and Attanasio and Low (2004).

${ }^{25}$ Instead, as noted by Chioda (2004), or Ventura (1994) for the parametric case, one can take differences of two adjacent Euler equations to form a valid moment condition and identify $\psi$.

${ }^{26}$ This equation also identifies the observable effect of measurement error $\delta_{C}$ for those elements of $K_{i, t}^{C}$ that change over time.
} 
Since $\psi$ and $\rho$ are known from the argument above, $b, \chi$, the remaining elements of $\delta_{C}$ and therefore $\delta_{H}$, as well as the distribution of $\xi_{i, T}^{C}$, are identified.

\subsection{Comparison to $\operatorname{ARMA}(1,1)$}

Many studies employ an $\operatorname{ARMA}(1,1)$ model for the unobservables in the wage equation, sometimes with additional restrictions (e.g., a random walk), and/or after taking out a fixed effect. ${ }^{27}$ In order to understand how this approach compares with our factor model approach, consider the time series process for a random variable $U_{t}$. Consider modeling the time series process of the unobserved variables using an $\operatorname{ARMA}(1,1)$ process, $U_{t}=v_{t}+\rho U_{t-1}+\phi v_{t-1}$. Replacing sequentially, we would have

$$
\begin{aligned}
& U_{1}=v_{1} \\
& U_{2}=(\rho+\phi) v_{1}+v_{2} \\
& U_{3}=\rho(\rho+\phi) v_{1}+(\rho+\phi) v_{2}+v_{3}
\end{aligned}
$$

By setting $v_{t}=\theta_{t}$ we can immediately see that both processes for $U_{t}$ are very similar, and that the factor decomposition we impose can nest the $\operatorname{ARMA}(1,1)$ model. ${ }^{28}$ The main difference is that the factor model implies less constraints on the loadings. ${ }^{29}$ Clearly, the approach we use to identify the agent's information set could also be applied in this case by checking whether $v_{t}$ affects choices at $\tau<t$.

\section{Data and Parametrization of the Model}

The model in this paper is estimated on a sample of white males who either graduated high school (and only high school) or are college graduates. Since the PSID data contains very few observations with measurements for ability (i.e., who took the IQ Word Test), and the NLSY79 does not contain the full lifecycle for any individual, the sample used contains individuals from both the NLSY79 and PSID datasets pooled together. ${ }^{30}$ The sample consists of a total of 1,642 white males born between 1923 and 1964, who either took the ASVAB battery of tests (NLSY) or the IQ Word Test (PSID). Of these, 1,263 come from the NLSY79 and 379 from the PSID.

Individual working life cycles are simplified to eight 6-year-long periods starting with age 18-23 and ending with age 60-65. ${ }^{31}$ This simplifying assumption is used to keep the computational complexity of the model manageable. For each period, hours worked is simply the sum of total hours worked during

\footnotetext{
${ }^{27}$ See Blundell et al. (2008); Gottschalk and Moffitt (1994); Heathcote et al. (2014); Lochner and Shin (2014); Meghir and Pistaferri (2004).

${ }^{28} \varepsilon_{t}=0, \forall t \leq 7, \varepsilon_{8}=v_{8} . \alpha_{11}=1 ; \alpha_{12}=\rho+\phi, \alpha_{22}=1 ; \alpha_{13}=\rho(\rho+\phi), \alpha_{23}=\rho+\phi, \alpha_{33}=1 ;$ etc.

${ }^{29}$ It also allows for an additional "transitory" shock, $\varepsilon$, every period; however, it is common to add an additional shock (e.g., measurement error) to the ARMA model.

${ }^{30}$ We are assuming that the environment is stationary since, conditional on observables and on including an NLSY dummy as a control in all equations, we treat both samples as comparable.

${ }^{31}$ There is an additional terminal period in the model which includes whatever happens after age 65 . Utility in this terminal period is modeled as depending only on assets carried to that period and a minimum income (e.g., social security income), so no additional data for the period is required.
} 
the six years. Log-earnings in the period are calculated as the log of the present value of earnings for the period discounted at a $3 \%$ annual rate. Wages for the period are then calculated as the ratio of earnings to hours worked in the period. Earnings and hours for individuals who are missing are imputed only in the years in which there was no survey using an average of the earnings in the years immediately adjacent (i.e., the year before and the year after) to the missing year. If earnings are not available in either of these years they are left as missing. Missings are treated as random events. ${ }^{32}$

The procedure to get consumption data in both samples is similar. Household consumption at time period $t$ is defined as the difference between available resources, household income plus assets available at the beginning of the period, minus the discounted assets available in the next period. In order to recover more consumption observations, if assets are not observed at the beginning of the period as required, we use assets either one year before or after it (discounted appropriately). Imputing household consumption for the PSID sample is done in the same way as with NLSY79, although questions about assets are not asked as frequently for this sample. To impute individual consumption, we divide household consumption by the square root of the number of members in the household. We account for the measurement error introduced by this procedure, by adding measurement error to our consumption equation (i.e., equation (13)) at estimation.

Tuition between 1972 and 2000 is defined as the average in-state tuition in colleges in the county of residence. If there is no college in the county, then average tuition in the state is taken instead. For years prior to 1972, national tuition trends are used to project county tuition backwards keeping the average observed structure of the period 1972-1977. ${ }^{33}$

Table 2 presents a summary of the pooled dataset used to estimate the model. In general, college graduates get higher wages, consume more, and have more assets than high school graduates. They also have higher test scores, come from better family backgrounds, have fewer siblings, and are more likely to live in a location where college tuition is lower.

For each schooling level $s=\{h s, c o l\}$ and for each period of wages $t=\{1, \ldots, 8\}$, we model $\ln W_{i, s, t}$ as being generated by a factor model:

$$
\ln W_{i, s, t}=X_{i, t} \beta_{s, t}+\sum_{j=1}^{\min \{t, 7\}} \theta_{i, j} \alpha_{s, t, j}+\varepsilon_{i, s, t} .
$$

The $X^{\prime} s$ include controls for age, a dummy for NLSY, and our generated (as described below) measure of ability, $Q_{i}$. As discussed in Section 3.3, we normalize $\alpha_{h, j, j}=1$ for $j=1, \ldots, 7$. The psychic cost function is also allowed to, in principle, be a function of all factors:

$$
P_{i}=Z_{i} \gamma+\sum_{j=1}^{7} \theta_{i, j} \lambda_{j}+\omega_{i}
$$

\footnotetext{
${ }^{32}$ See Fitzgerald et al. (1998) for evidence that observed people in PSID have similar characteristics as those in the CPS so attrition is roughly random. See MaCurdy et al. (1998) for evidence that attrition is roughly random in the NLSY79.

${ }^{33}$ We first run a regression of the difference between national tuition and county tuition between 1972 and 1977 against county dummies. The predicted value is the average difference between national and county tuition which can then be added to the national tuition observed in the years previous to 1972.
} 
Since this cost is perceived by the agent, it only depends those elements of $\theta_{i}$ in the agent's information set at the schooling decision age. The $Z_{i}$ include variables that only affect the schooling decision like family background, and local unemployment. Table 3 includes a complete list of the $X$ and $Z$ used in the wage and psychic cost equations.

In order to account for ability in a consistent way across both datasets, an additional one factor model that utilizes a system of external measurements on ability is used. In 1980, NLSY respondents were administered a battery of ten achievement tests referred to as the Armed Forces Vocational Aptitude Battery (ASVAB). (See Cawley et al. (1997) for a complete description.) The math and verbal components of the ASVAB can be aggregated into the Armed Forces Qualification Test (AFQT) scores. ${ }^{34}$ Many studies have used the overall AFQT score as a control variable, arguing that this is a measure of scholastic ability. In this paper, we interpret AFQT measures as imperfect proxies for scholastic ability, and the factor structure is used to capture this. Potential aggregation bias is avoided by using each of the components of the ASVAB score as a separate measure. PSID participants do not take the ASVAB battery of tests that NLSY participants do. Instead, we use the IQ Word test that was administered in 1972 and assume that this test (measured in percentiles) is comparable to the ASVAB tests (also in percentiles).

For the case of NLSY79 data, five components from the ASVAB battery of tests, measured as percentile ranks in the population are used. ${ }^{35}$ For PSID, the 1972 IQ test (also measured as percentile ranks) is included. Each test, $M_{i, j}$, is modeled as a function of individual ability, $Q_{i}$ :

$$
M_{i, j}=X_{i, j}^{M} \beta_{j}^{M}+Q_{i} \alpha_{j}^{M}+\varepsilon_{i, j}^{M}
$$

To pin down the scale and sign of ability, the loading on the arithmetic reasoning test $\left(\alpha_{1}^{M}\right)$ is normalized to 1 . This normalization associates higher levels of the factor with higher test scores, purged of the effect of $X^{M}$, so we interpret $Q_{i}$ as ability. In this interpretation, tests are assumed to be noisy proxies for ability which is given by $Q_{i}$. Identification of the loadings and non-parametric distributions of $Q_{i}, \varepsilon_{i, j}^{M}$ follows from similar arguments as those used in Appendix 1. Since we cannot directly relate the ASVAB tests to the IQ Word test (as no individual takes both tests), we need to make stronger assumptions about the distribution of $\varepsilon_{i}^{M}$ on the IQ Word test (see Online Appendix 2). The $X^{M}$ includes controls for age at test date, grade completed at test date, and whether the individual was enrolled in school at test date. Table 3 shows the full set of covariates used for the ability measures (as well as for, log wages, costs, and measurement errors in hours and consumption).

Each of the factors, $\theta_{i, j}$, is allowed to follow a mixture of mean zero normal distributions $\theta_{i, j} \sim$ $\sum_{\ell=1}^{L_{j}} \pi_{j, \ell} f\left(\theta_{i, j} ; \mu_{j, \ell}, 0.5\right)$, where $f\left(x ; \mu, \sigma^{2}\right)$ is a normal density with mean $\mu$ and variance $\sigma^{2} .{ }^{36}$ In all cases, mixtures with 2 elements are found to be adequate. The distribution of ability follows a mixture

\footnotetext{
${ }^{34}$ Implemented in 1950, the AFQT score is used by the U.S. Army to screen draftees.

${ }^{35}$ These are Arithmetic Reasoning, Word Knowledge, Paragraph Composition, Coding Speed and Math Knowledge. See also Hansen et al. (2004) for an analysis of ASVAB tests and their relation to ability.

${ }^{36}$ The fact that we normalize the variance of the individual components of the mixture does not mean we have fixed the variance of the factor, as the variance of a mixture is a function of all the parameters of the mixture. In principle we could have let the variance of each mixture to be a free parameter. We choose not to do so since this reduces the number of parameters to be estimated considerably, i.e., 14 less parameters.
} 
of three normals: $Q_{i} \sim \sum_{\ell=1}^{3} \pi_{Q, \ell} f\left(Q_{i} ; \mu_{Q, \ell}, \sigma_{Q, \ell}^{2}\right)$. The remaining distributions in the model (e.g., measurement errors, uniquenesses) are all assumed to be normal.

In order to allow for levels of borrowing that are roughly consistent with the ones observed in the data, we assume that individuals have access to some guaranteed income (e.g., social security income) when retired, $Y_{i, s, T+1}^{M I N}$. We set the levels of $Y_{i, s, T+1}^{M I N}$ to be roughly consistent with the 18 additional years of life expectancy at age 65 observed in the data, as well as with the average levels of social security income retiree's received in 2016: 1,341 monthly for high school graduates (974 in 2000 dollars), and 2,000 monthly for college graduates (1,453 in 2000 dollars). With these numbers, the maximum level of borrowing in each period is automatically determined by equation (3).

In our estimation, we set the maximum number of hours one can work, $\bar{H}_{s, t}$, to 24,000 hours per-period for all periods except $t=1$ in college. We only allow people who are in college in period 1 to work half time, and hence set $\bar{H}_{c o l, 1}$ to 12,000 in this case. We also approximate the (unobserved) distribution of initial assets (i.e., assets in period 0) with the distribution of non-negative assets in the first three periods (i.e., ages 18,24 and 30 ) multiplied times $0.5 .^{37}$

The model is estimated in three stages. In the first stage, we estimate the ability measurement model in equation (24) by maximum likelihood. ${ }^{38}$ Given the estimates, we predict $Q_{i}$ for all individuals and include it as part of the $X$ and $Z$ variables for the rest of the model. ${ }^{39}$

In the second stage, we estimate the model without solving for the optimal policies but rather using quadratic polynomials in the state variables for the semiparametric approximations to the policy functions in equations 17 and 18. We employ a similar polynomial as a semiparametric approximation to the schooling choice. In all cases we include the testing terms $\bar{\theta}_{i}(t) \pi_{t}$ as described in section 3.2. This step allows us to recover the information set of the agent, $\mathcal{I}_{i, t}$, that we employ in the final step. ${ }^{40}$

In the final step, we employ the information set of the agent recovered in the previous step to estimate the structural model. Estimation of the rest of the model is done by maximum likelihood using a combination of accelerated random search, the Nelder-Meade simplex method and the BFGS algorithm to maximize the likelihood. The contribution of individual $i$ who chooses schooling $S_{i}=s$ is

\footnotetext{
${ }^{37}$ We do not necessarily expect 17 year old individuals to actually have assets. However, we do not observe parental transfers in our data. One can think of our procedure as a way to account for parental transfers.

${ }^{38}$ The contribution to this likelihood for individual $i$ is$$
\int_{\mathcal{Q}} \prod_{j=1}^{J} f_{\varepsilon_{i, j}^{M}}\left(M_{i, j}-X_{i, j}^{M} \beta_{j}^{M}-Q_{i} \alpha_{j}^{M} \mid Q_{i}, X_{i, j}^{M}\right) d F(Q) .
$$

${ }^{39}$ The use of predicted $Q_{i}$ introduces "measurement" error as it is a generated regressor. In principle, we could correct our estimates ex-post as in Heckman et al. (2013). We could also include the likelihood in footnote (38) into our likelihood below and estimate everything in one step. Since, this would add 44 parameters and it would make our likelihood into a 9-dimensional integral, we decided to proceed this way.

${ }^{40}$ In principle we could have used the estimated wage equations from this step when we estimate the structural model under the information set we recover in the next step. To avoid issues of having two step estimators when calculating standard errors, we re-estimate the wage equations jointly with everything.
} 


$$
\int_{A_{0}, \Theta}\left[\begin{array}{c}
\prod_{t=1}^{8} f_{\varepsilon_{i, s, t}}\left(\ln W_{i, s, t}-X_{i, t} \beta_{s, t}-\theta_{i}^{\prime} \alpha_{s, t} \mid \theta_{i}, X_{i, s, t}\right) \\
\prod_{t=1}^{8} f_{\xi_{i, t}^{H}}\left(\ln \widehat{H}_{i, t}-\ln \mathscr{H}_{s, t}\left(\mathcal{I}_{i, t}\right)-\delta_{H}^{\prime} K_{i, t}^{H} \mid \theta_{i}, X_{i, s, t}, Z_{i}, K_{i, t}^{H}\right) \\
\prod_{t=2}^{8} f_{\xi_{i, t}^{C}}\left(\ln \widehat{C}_{i, t}-\ln \mathscr{C}_{s, t}\left(\mathcal{I}_{i, t}\right)-\delta_{C}^{\prime} K_{i, t}^{C} \mid \theta_{i}, X_{i, s, t}, Z_{i}, K_{i, t}^{C}\right) \\
\operatorname{Pr}\left(S_{i}=s \mid \theta_{i}, X_{i, s, t}, Z_{i}\right)
\end{array}\right] d F\left(A_{0}, \theta\right) .
$$

Evaluation of the likelihood requires that the econometrician solve the dynamic program in order to evaluate the schooling selection probability, the choice of hours worked, and the consumption policy function $\mathscr{C}_{s, t}$. Since the econometrician never observes any element of $\theta_{i}$, nor assets in period 0 , he has to integrate against their distribution when evaluating the likelihood. In the model we estimate, the value function given by the solution to the hours and consumption allocation problem is approximated numerically using a second order complete polynomial approximant.

\section{Empirical Results}

\subsection{Estimation and Fit}

We begin by estimating the model for ability of equation (24). We use five components of the ASVAB battery of tests contained in the NLSY79: Arithmetic Reasoning, Word Knowledge, Paragraph Composition, Coding Speed and Math Knowledge; as well as the 1972 IQ Word Test for PSID. Tables O1-1 and O1-2 in Online Appendix 1 contain the parameter estimates for this model. As expected, ability is associated with higher test scores. With the estimates in hand, we then use Bayes rule to predict ability for each individual. Figure 1 plots the distribution of predicted ability that we obtain using this procedure. As can be seen from the graph, the distribution is highly non-normal, hence it is important to allow for more general distributions like the mixture distribution we use.

Table 4 presents the results of the proposed test for the agent's information set using the auxiliary parameters, $\pi$, defined in Section 3.2 for the elements of $\bar{\theta}_{i}(t)$. We estimate all of the policy functions using a sieve for each function jointly with the model for wages as described in the previous section. In terms of the empirical model described above, this entails testing whether $\theta_{i, j}$ affects the agent's choices before period $j$ (when it hits wages).

In summary, the results of the test show that scholastic ability (i.e., $\left.Q_{i}\right),{ }^{41} \theta_{1}, \theta_{2}$, and $\theta_{7}$ are known at the time schooling choices are made; $\theta_{6}$ becomes known in period $1 ; \theta_{4}$ becomes known in period 2 ; finally all factors are known in period 3. As a consequence, all of the results presented in the next sections are based on estimates of the structural model using this information set.

Tables O1-3 to O1-7 in Online Appendix 1 present the parameter estimates for the model estimated under this information structure. In total the model has 144 parameters. ${ }^{42}$ The estimated parameters are all within reasonable ranges. In particular, we estimate a coefficient of risk aversion for consumption

\footnotetext{
${ }^{41}$ Cunha and Heckman (2016) also find ability is known at schooling time.

${ }^{42}$ The interest rate $r$ is set at $3 \%$ annually.
} 
of 0.49 (on the low side), a Frisch elasticity of labor of 1.00, and a discount factor of 0.94, which implies a yearly discount rate of around $1 \%$.

To validate the model estimates obtained under the information set we have identified, we next compare predictions of the model versus their data counterparts. First, the proportion of people who attend college in the data and as predicted by the model are compared. ${ }^{43}$ Whereas $48.5 \%$ of the people in the sample are college graduates, the model predicts roughly $48.3 \%$, where the null hypothesis of equality of predicted and actual proportions cannot be rejected with $5 \%$ significance.

Table 5 presents the (per-period) mean for the logs of wages, hours worked, and consumption both in the data as well as predicted from the model. Overall the model does a remarkably good job of matching the moments in the data. When we perform formal tests of equality between data and model means in Table 5, we cannot reject equality in any case. As we show in Tables O1-8 and O1-9 in Online Appendix 1 , the model fits the data on consumption and wages by schooling level equally well, but has a harder time reproducing the pattern of hours by schooling level, and the test of equality fails half the time.

As a final check on the predictions of the model, we compare the estimates of the wage return to college for college graduates, a treatment-on-the-treated style parameter, implied by our model estimates with estimates obtained using a semi-parametric control function approach as described in Navarro (2008). Our model estimates, by construction, are obtained under the assumptions that a) the errors can be factor analyzed, b) we have the right information set of the agent, and c) the consumption and labor supply decisions of the agent are correctly specified. Estimates based on the control function approach, on the other hand, do not impose any of these assumptions, and hence provide a benchmark against which to compare the wage return prediction of our model. ${ }^{44}$ We estimate a percentage gain of $72 \%$ with the control function, of $75 \%$ from the model, and the difference is not significant. We take this as evidence that our factor model assumptions are consistent with the data.

\subsection{Analysis of Results}

Sorting In Figures 2.1-2.4 we graph the densities of ability and factors 1, 2 and 7 conditioning on schooling choice. The remaining factors are not known by the agent at the time the schooling decision is made. Therefore, there is no selection based on them, and their distribution does not differ by schooling. There is strong evidence of selection in terms of ability, $\theta_{1}$, and $\theta_{7}$. The distribution of ability for college graduates is to the right of that for high school graduates. Individuals strongly sort in terms of ability, even after controlling for family background and individual characteristics at test date. People with higher ability graduate college more than people with lower ability. The average ability for high school graduates is 0.63 of a standard deviation lower than that of college graduates. A reverse pattern holds true for $\theta_{1}, \theta_{2}$ and $\theta_{7}$.

\footnotetext{
${ }^{43}$ All of our predicted results are based on simulating (i.e., sampling the unobservables) 50 times for each individual in the data.

${ }^{44}$ To obtain the control function estimates, we predict the probability of going to college with a probit of schooling on local unemployment, tuition, number of siblings, mothers education, and ability. We then run a regression of log wages on age, age-squared, ability, an indicator for NLSY, and a 2nd degree polynomial on the (log) probability of schooling obtained in the first step. We use these estimates to correct for selection bias, and calculate the wage gain of going to college for college attendees.
} 
Since the factors are not tied to an external set of measurements (like ability is), it is hard to provide an interpretation for them. In order to aid in interpretation, in Table 6 we present the correlation of ability, $\theta_{1}, \theta_{2}, \theta_{7}$, wages, hours, present value of earnings, and schooling choices. ${ }^{45}$ Ability is positively correlated with college wages, hours and earnings, as well as with college attendance, but uncorrelated with their high school counterparts. $\theta_{1}$, on the other hand, is positively correlated with high school wages, hours, and earnings, but negatively correlated with their college counterparts as well as with college attendance. $\theta_{2}$ is also positively correlated with high school outcomes and negatively with college outcomes, although in both cases the correlation is weaker than for $\theta_{1}$. $\theta_{2}$ is very weakly correlated with schooling. $\theta_{7}$, on the other hand, is very weakly correlated with wages but highly correlated with schooling choice. In summary, $\theta_{2}$ determines wages but not schooling, $\theta_{7}$ determines schooling but not (lifetime) wages, while $\theta_{1}$ and ability determine both.

Counterfactual Gains Figures 3.1 and 3.2 compare fitted and counterfactual distributions of log earnings for each schooling level. Let $P V_{i, h s}$, denote the potential present value of earnings in high school for individual $i$, regardless of which schooling level he actually attains. Figure 3.1 compares $f\left(\log P V_{h s} \mid s=h s\right)$ with $f\left(\log P V_{h s} \mid s=c o l\right)$, i.e., high school earnings for actual high school graduates (a "factual" distribution), and potential high school earnings for actual college graduates (a "counterfactual" distribution). Figure 3.2 does the same for potential college earnings. The figures show that high school earnings for people who actually stop at high school are higher than high school earnings would have been for college graduates (had they stopped at high school instead). In a similar manner, college graduates have higher college earnings than high school graduates would have had they graduated college instead. So, at least in terms of earnings, people seem to be sorting in the expected direction.

In Figure 3.3 we plot the difference in log lifetime earnings, (i.e., the ex-post realized gain of attending college, implied by the previous figures). Consistent with the estimated sorting shown, the average annual return to college for high school graduates (i.e., treatment-on-the-untreated) is $-1.6 \%$, while the annual return for college graduates is $20.5 \% .{ }^{46}$ Two additional features are worth noting. First, a large proportion of high school graduates would have obtained positive ex-post gains if they had gone to college. Second, a considerable fraction of college graduates earn an ex-post negative gain. From our analysis above, we know that ability and $\theta_{1}$ are most important for explaining the difference in Figure 3.3 between high school and college graduates. That is, the difference is largely due to the different compositions in terms of scholastic ability and $\theta_{1}$ via their effects on preferences for schooling and on wages.

Variability and Uncertainty In order to clarify the difference between variability and uncertainty, Table 7 presents the average (over periods) variance of log wages under three different assumed information sets for the agent at the time the schooling decision is made. Even though there is a lot of dispersion, not all of it is truly uncertain to the agent. In particular, under the estimated information set at time 0 ,

\footnotetext{
${ }^{45}$ We predict wages and hours over the lifecycle for each individual for each schooling level, regardless of schooling level chosen. We use these predicted wages and hours to calculate the present value of earnings. We then calculate the correlation of all of these components.

${ }^{46}$ All annual figures are calculated assuming it takes 4.5 years to get a college degree.
} 
roughly $56 \%(52 \%)$ of what would typically (in other studies) be considered uncertainty in high school (college) wages is predictable by the agent at the time his schooling decision is made. In the same manner, the variance of the wage gains to college when the information set contains $\theta_{1}, \theta_{2}$ and $\theta_{7}$ is only $33 \%$ of what we would obtain if we assumed the agent knows no more than the analyst. Of the total observed variability in high school log wages, only $44 \%$ (i.e., $0.2092 / 0.4754$ ) constitutes true uncertainty for the individual, i.e., variability not explained by his information. Similarly for college where $48 \%$ is left unexplained.

Preferences The estimated risk aversion coefficient in the model is 0.5 . While this number is on the low end of the numbers reported by Browning, Hansen, and Heckman (1999), agents are risk averse and do not care only about monetary returns.

In Figure 4.1 we plot the difference in the value function of attending college vs. not attending, ignoring the psychic cost, as perceived by the agent at the time the schooling decision is made. Even though most people who choose to go to college have a higher gross utility return to college than high school graduates, many do not. That is, we still find people who choose college with negative gross differences in the lifetime expected utility from consumption and leisure, and people who choose high school with positive differences. As shown in Figure 4.2, this discrepancy is captured by the difference in preferences for schooling (the "psychic" cost function). People with low psychic costs (i.e., people who have a preference for school) are more likely to finish college. This preference is mostly driven by ability (since people with higher ability tend to have lower psychic costs) and by $\theta_{1}$, and to a lesser extent by $\theta_{7}$.

Credit constraints, risk aversion and uncertainty Separating the effect of borrowing constraints from uncertainty is not an easy task given the model we have estimated. For one thing, the CRRA preferences that we estimate have a single parameter that measures both risk aversion and intertemporal substitution. Furthermore, by construction, the effect that the borrowing constraint has on the agent's behavior is directly related to the uncertainty faced by the agent. In an attempt to separate the effects of these two components, we perform two simulations.

In the first simulation, presented in line 2 of Table 8 , we eliminate uncertainty in wages, keeping the credit constraints in effect. In order to do so, we assume that the agents have perfect certainty about what their wages will be in the future. We keep the constraints at the original level in order to study the importance of uncertainty alone, even though the model implies that borrowing limits and uncertainty are directly linked. In the aggregate, college attendance increases by $5.7 \%$-points, from $48.3 \%$ to $54.0 \%$.

Table 9 also shows the importance of looking at micro evidence when accounting for uncertainty in schooling decisions. At the individual level, there is a large difference between decisions made ex-ante and those made under perfect certainty. Roughly $21 \%$ of the individuals who, under uncertainty, choose to stop at high school would choose to graduate college if uncertainty were removed. Roughly $11 \%$ of college graduates would have stopped at high school under complete certainty. The micro findings are consistent with the patterns of Figure 3.3: a proportion of high school graduates would have earned positive ex-post gains had they gone to college, and a fraction of college graduates would earn negative ex-post gains and so may have chosen not to go to college if they had known. 
In the second simulation, borrowing limits, and the effects of uncertainty, are both eliminated. We allow them to operate in an environment equivalent to complete markets in the third line of Table 8 . The effects are significantly different from those obtained from just eliminating uncertainty as shown in the second line of Table 8 . When we also complete the market by eliminating the borrowing limit, college attendance increases an additional 5.3\%-points. We call this change the "pure" effect of eliminating the borrowing limit. Compared to the case in which only uncertainty is eliminated, letting people smooth intertemporal consumption with complete markets, allows individuals to maintain desired consumption while in college and at young age. As a consequence, most of the changes in schooling choices come from high school graduates deciding to continue on to college as shown in Table 10. Only a very small number of college graduates would have chosen not to go to college under complete markets.

\section{The importance of correctly accounting for uncertainty: adverse se- lection in a policy example}

To illustrate the importance of accounting for the right information set and the information structure of the model, in this section we introduce a new environment. We assume that an institution, which for simplicity we call a bank, offers a minimum wage $(\underline{W})$ insurance contract to the agent. While we do not explicitly link this contract with loans for school, its design contains some of the features listed by Lochner and Monge-Naranjo (2016) as desirable in an optimal student loan contract. In particular, the insurance premium depends on expected income and it offers larger transfers to agents experiencing bad post-school outcomes.

We denote the bank's information set about individual $i$ (which may or may not coincide with that of the agent) by $\mathcal{J}_{i}$. We assume that the bank offers the agent a one-time contract right before he makes his schooling choice. The contract specifies a premium, $m_{i, s}\left(\mathcal{J}_{i}\right)$, which is schooling specific and depends on the information the bank has on the agent. The (future value of the) premium is paid in the last period $t=T+1$, so it is like a loan to be paid upon retirement. In order to keep the problem simple, we assume that the bank uses the following rule to determine the premium.

We assume that the bank assumes that the agent has the same information that it does. It sets the premium equal to its expected present value of cost under the worst case scenario for the bank, i.e., when the agent faces market wages below the insured wage all his life, and hence continually uses the insurance to guarantee his minimum wage:

$$
m_{i, s}\left(\mathcal{J}_{i}\right)=\sum_{t=1}^{T} \frac{1}{(1+r)^{t-1}} \int_{0}^{\underline{W}}\left(\underline{W}-W_{i, s, t}\right) H_{i, t} f\left(W_{i, s, t} \mid \mathcal{J}_{i}\right) d W_{i, s, t} .
$$

The premium is such that, under this "worst-case-scenario" for the bank, it gives zero expected profits. In order to calculate the premium, however, the bank needs to solve the problem of the agent to calculate what $H_{i, t}$ the agent chooses when faced with the possibility of buying the insurance. That is, for each 
schooling level $s$, the bank solves

$$
\begin{aligned}
\max _{c_{i, t}, H_{i, t}} & \sum_{t=1}^{T} \beta^{t-1}\left(u\left(c_{i, t}, H_{i, t}\right)\right)+\beta^{T} \tilde{u}\left(c_{i, T+1}\right) \\
\text { s.t. } & \sum_{t=1}^{T} \frac{c_{i, t}-\underline{W} H_{i, t}}{(1+r)^{t-1}}+\frac{c_{i, T+1}-Y_{i, s, T+1}^{M I N}}{(1+r)^{T}}=A_{i, 0}(1+r)+\mathbb{1}(t=1, s=c o l) D_{i}+m_{i, s}\left(\mathcal{J}_{i}\right) .
\end{aligned}
$$

With the premium in hand, the bank can calculate the borrowing limits implied by the repayment constraint, as well as the new $Y_{i, s, t}^{M I N}$ given by the minimum wage and the labor supply decision of the agent. Individuals decide whether to go to college or not, and whether to buy the insurance and pay the premium at period $T+1$.

We simulate the model under three different scenarios related to different information sets between individuals and the bank. In all scenarios, both the bank and the agent are assumed to know the $X^{\prime} s$, $Z^{\prime} s$, ability, tuition fees, and the initial asset levels, but not the $\varepsilon^{\prime} s$ in the wage equations. In the first scenario, which we will call the "no information" case, both the bank and the agent have the same "naive" information set at $t=0$, where neither the bank nor the agent knows any of the factors at $t=0$ and they assume they will only learn them each period as they affect wages. In the second scenario, which we call the "asymmetric information" case, the agent has access to the information set we estimate in the previous section (i.e., he knows $\theta_{i, 1}, \theta_{i, 2}$, and $\theta_{i, 7}$ at $t=0$ ) but the bank still does not know any of the factors for any agent and assumes the agent has the same information that the bank does. In the final scenario, which we call the "equal information" case, we let the agent and the bank both have access to the information set we estimated in the previous section. These are summarized in Table 11.

Table 11

\begin{tabular}{|cc|c|c|}
\hline \hline \multicolumn{2}{|c|}{ Scenario } & Bank & Agent \\
\hline no information & $\mathcal{J}_{i}=\mathcal{I}_{i, 0}$ & $\theta_{i} \not \subset \mathcal{J}_{i}$ & $\theta_{i} \not \subset \mathcal{I}_{i, 0}$ \\
\hline asymmetric information & $\mathcal{J}_{i} \neq \mathcal{I}_{i, 0}$ & $\theta_{i} \not \subset \mathcal{J}_{i}$ & $\theta_{i} \cap \mathcal{I}_{i, 0}=\left\{\theta_{i, 1}, \theta_{i, 2}, \theta_{i, 7}\right\}$ \\
\hline equal information & $\mathcal{J}_{i}=\mathcal{I}_{i, 0}$ & $\theta_{i} \cap \mathcal{J}_{i}=\left\{\theta_{i, 1,1} \theta_{i, 2}, \theta_{i, 7}\right\}$ & $\theta_{i} \cap \mathcal{I}_{i, 0}=\left\{\theta_{i, 1,1}, \theta_{i, 2}, \theta_{i, 7}\right\}$ \\
\hline
\end{tabular}

These three scenarios enable us to evaluate the importance of different aspects of market information. We look at effects on individual choices: education, consumption, and labor supply; on consumer welfare; and on bank profits. By comparing either the no information scenario or the equal information scenario with the asymmetric information scenario, we can evaluate the effect of adverse selection, in particular on bank profits. By comparing the no information scenario with the equal information one, we can evaluate the effects of having access to more information overall. Overall, the simulations show that assumptions about the information set of the agent (and the bank) have a very large impact on the choices made by the agents, both in terms of schooling, labor, and consumption; and in terms of what types of agents buy insurance. Furthermore, the amount and asymmetry of information available is crucial as it determines the importance of adverse selection, and the possibilities for better screening through changes in premiums. As we show below, these information differences can lead to important gains (and losses) both for the consumer (in terms of welfare) and for the bank (in terms of profits). 
Sorting We begin by analyzing the patterns of selection in terms of ability and $\left\{\theta_{1}, \theta_{2}, \theta_{7}\right\}$ for a $\$ 12.5$ value of the minimum wage insurance in Table $12 .{ }^{47}$ In the no information scenario, there is no differential sorting based on the factors between agents who buy insurance and those who do not. In this case, neither the premium nor the agent choices reflect these factors as they are unknown to both the bank and the agent. Once we assume the agent has access to his estimated information set, there are clear selection patterns.

In Figures 5(a)-(c) we show the pattern of selection for each factor across two groups who make different choices under the asymmetric information and the equal information scenarios: those who buy insurance in the asymmetric information case but not in the equal information case, and those who do not buy insurance under asymmetric information but do under equal information. For the case of factor $\theta_{1}$, when we move to the equal information case people with low values of $\theta_{1}$ start buying insurance, and the people with high values of $\theta_{1}$ stop buying it. Sorting also occurs on $\theta_{2}$ (to a lesser extent) and on $\theta_{7}$. The role played by these factors in wages highlights the role of adverse selection due to the information asymmetries. Since originally the agents know $\left\{\theta_{1}, \theta_{2}, \theta_{7}\right\}$ but the bank does not, the figures reflect that by eliminating the information asymmetry the bank can effectively adjust the premium and eliminate the adverse selection, i.e., agents with values of these factors that make them more likely to have low wages in the future are now less likely to buy insurance. To see why this is the case, in Figure 6 we plot the premium differences for those who purchase the insurance under asymmetric information, but not under equal information. The main reason these agents no longer purchase the minimum wage insurance is that their premium went up now that the bank has access to the same information. Hence, as the bank observes the same information as the agent, there will be no adverse selection.

Consumption and Labor Supply In Tables 13 and 14 we show how the minimum wage insurance affects the consumption and labor supply choices of the agent over life-cycle. In the tables we examine the extent to which insurance affects these choices for those who buy the insurance when available. Overall, agents consume more when there is insurance for all three scenarios. A similar finding holds for labor supply. As insurance affects mostly the left tail of the wage distribution, the income effect of higher wages dominates and, overall, agents work more when there is insurance. The pattern is especially true for the early and later periods of the life cycle.

Schooling We next compare the agents' college attendance decisions with and without minimum wage insurance in Table 15. When the minimum wage is guaranteed, more agents choose not to attend college. This implies that, for some individuals, education is playing an important insurance role against adverse wage shocks. Once minimum wage insurance is available, these individuals choose not to attend college. Since the downside risk for high school wages is larger, by limiting the individuals exposure to this risk, minimum wage insurance makes high school more attractive for those individuals more exposed to this risk.

In Table 16, we analyze this pattern in more detail. Overall the change in college attendance associated

\footnotetext{
${ }^{47}$ We find similar patterns when we set it to $\$ 10$ or $\$ 15$ per hour.
} 
with the different scenarios is modest. $50.2 \%$ will go to college in the no information case, $47.8 \%$ in the asymmetric information case, $47.5 \%$ in the equal information case. There is, however, a lot of switching going on behind those changes. When we compare the no information case to the equal information case, to isolate the effect of more information from the adverse selection, we can see that around $40 \%$ of agents will switch from high school to college, and a similar percentage chooses high school instead of college. Compared to the change from the asymmetric information scenario to the equal information of around $1 \%$ both ways, we can see that adverse selection has a much smaller effect on schooling choices than having access to the additional information we estimate.

Welfare and Profits We next examine consumer welfare under the three information scenarios. In Figure 7, we plot the distribution of consumer life-cycle welfare (utility). Overall, we find that there is a large welfare gain associated with giving the consumer the right information set (i.e., switching from the no information to the equal information scenario), while eliminating adverse selection does not seem to have a large impact. In Table 17 we show that there is roughly a $28 \%$ gain on average welfare when we give the agent more information, and that this is not affected by asymmetric information.

Finally, in Table 17 we also compare the average bank profits for each scenario. The most striking pattern is the large negative effect that adverse selection can have on bank profits. When adverse selection exists (the asymmetric information scenario), the bank has negative profits on average. Profits, on the other hand, are similar when there is no asymmetric information regardless of the information structure. In Figure 8, we plot the distribution of bank profits over the population. As expected, when there is asymmetric information, the individuals who are more likely to have low wages prefer to buy insurance, so the bank profits distribution shifts left. On the other hand, the effect of increasing the banks access to information is to compress the profit distribution.

\section{Conclusion}

As emphasized by Lochner and Monge-Naranjo (2016), the optimal design of policies to finance schooling requires the capacity to identify what represents ex-ante risk to the individual. This requirement is common to many applied policy problems. Our paper develops a strategy that allows the researcher to distinguish what is unknown by the agent from what is unobserved by the econometrician using observational data. This allows the researcher to recover this ex-ante risk separately from unobserved heterogeneity and other factors known by individuals when schooling decisions are made.

We show how to implement our strategy using a factor decomposition of wage residuals to model uncertainty. Our estimated results illustrate the importance of properly accounting for what is unknown to the agent. For example, we find that $52 \%$ and $56 \%$ of the variance of college and high school log wages respectively are predictable by the agent at the time schooling choices are made. When we complete the market (by eliminating risk and borrowing limits), college attendance increases from $48 \%$ to $59 \%$. About half of this increase comes from eliminating uncertainty.

We also simulate a minimum wage insurance policy that contains some of the features that Lochner 
and Monge-Naranjo (2016) list as desirable in an optimal contract. We simulate the effects of the insurance contract under different assumptions about the uncertainty facing the agent and the institution offering the insurance. Our results show how adverse selection can unravel an otherwise profitable insurance market. Furthermore, we show that the implications of minimum wage insurance for consumption, labor supply, and schooling choices, depend a lot on the information that the individual has.

There are many directions in which one might consider extending the strategy we propose in this paper. As a conclusion, we provide an example of a possible extension. Since we do not know the banks information set, our policy simulations are simple illustrations of what could happen depending on how the information available to the agent and to the bank differs. If one had information on the characteristics of the contracts offered by the bank, this could be used to infer the bank's information about the agent using a similar strategy to that used for the agent. One could also use our approach to design efficient contracts given the estimated risk faced by agents. 


\section{References}

Aguirregabiria, V., Mira, P., 2002. Swapping the nested fixed point algorithm: A class of estimators for discrete markov decision models. Econometrica 70 (4), 1519-1543.

Aiyagari, S. R., August 1994. Uninsured idiosyncratic risk and aggregate saving. Quarterly Journal of Economics 109 (3), 659-684.

Attanasio, O., Low, H., 2004. Estimating euler equations. Review of Economic Dynamics 7, 405-435.

Belzil, C., Hansen, J., September 2002. Unobserved ability and the return to schooling. Econometrica 70 (5), 2075-2091.

Billingsley, P., 1995. Probability and measure, 3rd Edition. A Wiley-Interscience publication. Wiley, New York.

Blundell, R., Pistaferri, L., Preston, I., 2008. Consumption inequality and partial insurance. The American Economic Review 98 (5), 1887-1921.

Bonhomme, S., Robin, J.-M., April 2010. Generalized non-parametric deconvolution with an application to earnings dynamics. Review of Economic Studies 77 (2), 491-533.

Brown, M., Scholz, J. K., Seshadri, A., May 2012. A new test of borrowing constraints for education. Review of Economic Studies 79 (2), 511-538.

Browning, M., Hansen, L. P., Heckman, J. J., December 1999. Micro data and general equilibrium models. In: Taylor, J. B., Woodford, M. (Eds.), Handbook of Macroeconomics. Vol. 1A. Elsevier, Ch. 8, pp. $543-633$.

Browning, M., Lusardi, A., December 1996. Household saving: Micro theories and micro facts. Journal of Economic Literature 34 (4), 1797-1855.

Cameron, S. V., Heckman, J. J., June 2001. The dynamics of educational attainment for black, hispanic, and white males. Journal of Political Economy 109 (3), 455-99.

Cameron, S. V., Taber, C., February 2004. Estimation of educational borrowing constraints using returns to schooling. Journal of Political Economy 112 (1), 132-182.

Carneiro, P., Hansen, K., Heckman, J. J., May 2003. Estimating distributions of treatment effects with an application to the returns to schooling and measurement of the effects of uncertainty on college choice. International Economic Review 44 (2), 361-422, 2001 Lawrence R. Klein Lecture.

Carneiro, P., Heckman, J. J., October 2002. The evidence on credit constraints in post-secondary schooling. Economic Journal 112 (482), 705-734.

Carranza, J. E., 2007. Product innovation and adoption in market equilibrium: The case of digital cameras, unpublished manuscript, University of Wisconsin-Madison, Department of Economics. 
Cawley, J., Conneely, K., Heckman, J. J., Vytlacil, E. J., 1997. Cognitive ability, wages and meritocracy. In: Devlin, B., Fienberg, S., Resnick, D., Roeder, K. (Eds.), Intelligence, Genes, and Success : Scientists Respond to The Bell Curve. Springer, New York, pp. 179-192.

Cawley, J., Heckman, J. J., Lochner, L. J., Vytlacil, E. J., 2000. Understanding the role of cognitive ability in accounting for the recent rise in the return to education. In: Arrow, K., Bowles, S. (Eds.), Meritocracy and Economic Inequality. Princeton University Press, Princeton, NJ.

Chatterjee, S., Ionescu, F., 2012. Insuring student loans against the financial risk of failing to complete college. Quantitative Economics 3 (3), 393-420.

Chioda, L., 2004. Estimating euler equations with measurement error: A nonparametric approach, unpublished Manuscript, University of California Berkeley, Department of Economics.

Cooley Fruehwirth, J., Navarro, S., Takahashi, Y., October 2016. How the timing of grade retention affects outcomes: Identification and estimation of time-varying treatment effects. Journal of Labor Economics $34(4)$.

Cunha, F., Heckman, J., 2016. Decomposing trends in inequality in earnings into forecastable and uncertain components. Journal of labor economics 34 (S2), S31-S65.

Cunha, F., Heckman, J. J., Navarro, S., April 2005. Separating uncertainty from heterogeneity in life cycle earnings, the 2004 Hicks lecture. Oxford Economic Papers 57 (2), 191-261.

Fitzgerald, J., Gottschalk, P., Moffitt, R., 1998. An analysis of sample attrition in panel data: The michigan panel study of income dynamics. Journal of Human Resources 33 (2), 251-299.

Flavin, M. A., October 1981. The adjustment of consumption to changing expectations about future income. Journal of Political Economy 89 (5), 974-1009.

French, E., Taber, C., 2011. Identification of models of the labor market. Handbook of Labor Economics $4,537-617$.

Gottschalk, P., Moffitt, R., 1994. The growth of earnings instability in the U.S. labor market. Brookings Papers on Economic Activity 2, 217-254.

Gourinchas, P.-O., Parker, J., January 2002. Consumption over the lifecycle. Econometrica 70 (1), 47-89.

Hai, R., Heckman, J. J., 2016. Inequality in human capital and endogenous credit constraints. Working Paper.

Hansen, K. T., Heckman, J. J., Mullen, K. J., July-August 2004. The effect of schooling and ability on achievement test scores. Journal of Econometrics 121 (1-2), 39-98.

Hansen, L. P., Singleton, K. J., 1983. Stochastic consumption, risk aversion, and the temporal behavior of asset returns. Journalof Political Economy 91 (2), 249-265. 
Heathcote, J., Storesletten, K., Violante, G. L., 2014. Consumption and labor supply with partial insurance: An analytical framework. The American Economic Review 104 (7), 2075-2126.

Heckman, J., Pinto, R., Savelyev, P., 2013. Understanding the mechanisms through which an influential early childhood program boosted adult outcomes. The American economic review 103 (6), 2052-2086.

Heckman, J. J., Navarro, S., February 2007. Dynamic discrete choice and dynamic treatment effects. Journal of Econometrics 136 (2), 341-396.

Hotz, V. J., Miller, R. A., July 1993. Conditional choice probabilities and the estimation of dynamic models. Review of Economic Studies 60 (3), 497-529.

Huggett, M., September-November 1993. The risk-free rate in heterogeneous-agent incomplete-insurance economies. Journal of Economic Dynamics and Control 17 (5-6), 953-969.

Kane, T. J., Spring 1996. College cost, borrowing constraints and the timing of college entry. Eastern Economic Journal 22 (2), 181-194.

Kaufmann, K., Pistaferri, L., 2009. Disentangling insurance and information in intertemporal consumption choices. American Economic Review 99 (2), 387-92.

Keane, M. P., Todd, P. E., Wolpin, K. I., 2011. The structural estimation of behavioral models: Discrete choice dynamic programming methods and applications. Handbook of Labor Economics 4, 331-461.

Keane, M. P., Wolpin, K. I., June 1997. The career decisions of young men. Journal of Political Economy $105(3), 473-522$.

Keane, M. P., Wolpin, K. I., November 2001. The effect of parental transfers and borrowing constraints on educational attainment. International Economic Review 42 (4), 1051-1103.

Kotlarski, I. I., 1967. On characterizing the gamma and normal distribution. Pacific Journal of Mathematics 20, 69-76.

Lochner, L. J., Monge-Naranjo, A., October 2011. The nature of credit constraints and human capital. American Economic Review 101 (6), 2487-2529.

Lochner, L. J., Monge-Naranjo, A., 2016. Student loans and repayment: Theory, evidence, and policy. In: Eric A. Hanushek, S. M., Woessmann, L. (Eds.), Handbook of the Economics of Education. Vol. 5. Elsevier, Amsterdam, pp. 397 - 478.

Lochner, L. J., Shin, Y., 2014. Understanding earnings dynamics: Identifying and estimating the changing roles of unobserved ability, permanent and transitory shocks. Tech. rep., National Bureau of Economic Research.

MaCurdy, T. E., Mroz, T., Gritz, R. M., Spring 1998. An evaluation of the national longitudinal survey on youth. Journal of Human Resources 33 (2), 345-436. 
Marschak, J., 1953. Economic measurements for policy and prediction. In: Hood, W., Koopmans, T. (Eds.), Studies in Econometric Method. Wiley, New York, pp. 1-26.

Matzkin, R. L., March 1992. Nonparametric and distribution-free estimation of the binary threshold crossing and the binary choice models. Econometrica 60 (2), 239-270.

Matzkin, R. L., September 2003. Nonparametric estimation of nonadditive random functions. Econometrica 71 (5), 1339-1375.

Meghir, C., Pistaferri, L., 2004. Income variance dynamics and heterogeneity. Econometrica 72 (1), 1-32.

Navarro, S., 2008. Control function. In: Durlauf, S. N., Blume, L. E. (Eds.), The New Palgrave Dictionary of Economics., 2nd Edition. Palgrave Macmillan Press, London.

Peterman, W. B., 2016. Reconciling micro and macro estimates of the frish labor supply elasticity. Economic Inquiry 54 (1), 100-120.

Pistaferri, L., August 2001. Superior information, income shocks, and the permanent income hypothesis. Review of Economics and Statistics 83 (3), 465-476.

Prakasa Rao, B., 1992. Identifiability in Stochastic Models: Characterization of Probability Distributions. Probability and mathematical statistics. Academic Press, Boston.

Rogerson, R., Shimer, R., 2011. Search in macroeconomic models of the labor market. Handbook of Labor Economics 4, 619-700.

Sims, C. A., September 1972. Money, income, and causality. American Economic Review 62 (4), 540-552.

Storesletten, K., Telmer, C. I., Yaron, A., 2004. Consumption and risk sharing over the life cycle. Journal of monetary Economics 51 (3), 609-633.

Taber, C. R., July 2001. The rising college premium in the eighties: Return to college or return to unobserved ability? Review of Economic Studies 68 (3), 665-691.

van der Klaauw, W., 2012. On the Use of Expectations Data in Estimating Structural Dynamic Choice Models. Journal of Labor Economics 30 (3), 521 - 554.

Ventura, E., 1994. A note on measurement error and euler equations. Economics Letters 45, $305-308$.

Zhang, H. H., 1997. Endogenous borrowing constraints with incomplete markets. The Journal of Finance $52(5), 2187-2209$. 


\section{Appendix 1: Semiparametric Identification}

The following assumptions are used throughout this section in order to prove semiparametric identification of all the elements of the model. For simplicity, delete the $i$ subscript. Let $U_{s}=\left(U_{s, 1}, \ldots, U_{s, T}\right), U=$ $\left(U_{h s}, U_{c o l}\right), \xi^{H}=\left(\xi_{1}^{H}, \ldots, \xi_{T}^{H}\right), \xi^{C}=\left(\xi_{1}^{C}, \ldots, \xi_{T}^{C}\right), \xi=\left(\xi^{H}, \xi^{C}\right)$.

(A-1) $U, \zeta$ and $\xi$ have distributions that are absolutely continuous with respect to Lebesgue measure with support Support $(U) \times$ Support $(\zeta) \times$ Support $(\xi)$ that may be bounded or infinite. Variances are assumed to be finite. The cumulative distribution function of $\zeta$ is assumed to be strictly increasing over its full support. ${ }^{48}$

$$
(\mathbf{A - 2})(X, K, Z) \Perp(U, \zeta, \xi)
$$

\section{Semiparametric Identification of Log-wages with and without a Factor Structure}

Identification of the log-wage equations is proved in theorem $1 .{ }^{49}$ Only the case in which the measurements are continuous is considered, but, as shown in Carneiro et al. (2003), the measurements could also be discrete or mixed discrete-continuous. ${ }^{50}$

Theorem 1. Let $\mu_{s}(X)=\left(\mu_{s, 1}(X), \ldots, \mu_{s, T}(X)\right)$. Assume that the relevant elements of $(\boldsymbol{A}-1)$ and (A-2) (i.e., the joint conditions on $X, Z, U, \zeta)$ hold and that the following variation free condition holds:

(A-3) Support $\left(\phi(Z), \mu_{s}(X)\right)=\operatorname{Support}(\phi(Z)) \times \operatorname{Support}\left(\mu_{s}(X)\right)$. (Variation free)

Assume that Support $(\phi(Z)) \supseteq \operatorname{Support}(\zeta)$ and $\operatorname{Support}\left(\mu_{s}(X)\right) \supseteq \operatorname{Support}\left(U_{s}\right)$. Then, the mean functions $\mu_{s, t}(X)$ are identified on the support of $X$. Also, the joint distribution of $U_{s}$ is nonparametrically identified for $t=1, \ldots, T$ for each $s=h s$, col.

Proof. Under the conditions of the theorem, we can find limit sets $\mathcal{Z}^{-}$and a $\mathcal{Z}^{+}$such that $\operatorname{Pr}\left(S=\operatorname{col} \mid Z \in \mathcal{Z}^{-}\right)=0$ and $\operatorname{Pr}\left(S=\operatorname{col} \mid Z \in \mathcal{Z}^{+}\right)=1$ where we can still freely change the $\mu_{s}(X)$. Identification of the mean functions over their support is trivial since we observe $\ln W_{s}$ for each $X$ and can recover the marginal distribution of $U_{s}$. The intercepts are recovered from assumed zero mean of $U_{s}$. The joint follows immediately since $\operatorname{Pr}\left(\ln W_{s}<w \mid X\right)=F_{U_{s}}\left(w-\mu_{s}(X)\right)$ by assumption (A-2). Then, from (A-3) we can find an $X=x$ where $\mu_{s}(x)=k$ and $k$ is a $T$ dimensional vector. Let $m=k-\mu_{s}(x)$ so $\operatorname{Pr}\left(\ln W_{s}<w \mid X=x\right)=F(k)$. Since the point $w$ is arbitrary, we can vary it to identify the full joint distribution.

When the unobservables are represented in terms of equations (14), the next theorems show that we can nonparametrically identify the distributions of the factors and the uniquenesses as well as the factor loadings. Consider using only the information wages in each schooling state. For a given schooling level

\footnotetext{
${ }^{48}$ This assumption can easily be relaxed and is only made for convenience.

${ }^{49}$ See Heckman and Navarro (2007).

${ }^{50}$ In all cases, with additional assumptions, we can relax additive separability and identify functions of the form $y=$ $\mu(X, U)$ by using the analysis in Matzkin (2003).
} 
$s$ we have a system of equations

$$
\begin{gathered}
\ln W_{s, 1}=\mu_{s, 1}\left(X_{s, 1}\right)+\theta^{\prime} \alpha_{s, 1}+\varepsilon_{s, 1} \\
\vdots \\
\ln W_{s, T}=\mu_{s, T}\left(X_{s, T}\right)+\theta^{\prime} \alpha_{s, T}+\varepsilon_{s, T}
\end{gathered}
$$

The total number of equations is given by $T$, while the total number of factors is given by $T-1$ (an additional factor per-period up to $T-1$ ). Such an arrangement would be motivated by the assumptions about the arrival of information made in the text.

\section{Identifying loadings - non-symmetric distributions}

We continue the argument in the main text for the case of non-symmetric distributions where we show how to recover the loadings for the first two periods, as well as the second and third moments of the first two elements of $\theta$ and the uniquenesses. By forming

$$
\frac{E\left(U_{h s, t} U_{h s, t+1}^{2}\right)-\sum_{j=1}^{t-1} \alpha_{h s, t, j} \alpha_{h s, t+1, j}^{2} E \theta_{j}^{3}}{E\left(U_{h s, t}^{2} U_{h s, t+1}\right)-\sum_{j=1}^{t-1} \alpha_{h s, t, j}^{2} \alpha_{h s, t+1, j} E \theta_{j}^{3}}=\alpha_{h s, t+1, t}
$$

we can recover the loading for factor $t$ in period $t+1$. $E\left(\theta_{t}^{2}\right)$ can be recovered from $E\left(U_{h s, t} U_{h s, t+1}\right)$. Having $E\left(\theta_{t}^{2}\right)$ in hand, we can identify $\alpha_{h s, j, t}$ from $E\left(U_{h s, t} U_{h s, j}\right), j>t+1$. From $E\left(U_{h s, t}^{2}\right)=\sum_{j=1}^{t} \alpha_{h s, j, t} E \theta_{j}^{2}+$ $E \varepsilon_{h s, t}^{2}$, we recover $E \varepsilon_{h s, t}^{2}$. Finally, $E \theta_{t}^{3}$ can be identified from $E\left(U_{h s, t}^{2} U_{h s, t+1}\right)$, and $E \varepsilon_{h s, t}^{3}$ from $E\left(U_{h s, t}^{3}\right)$. Since we cannot separately identify $\theta_{T}$ from $\varepsilon_{h s, T, T}$, as there is no $T, T+1$ pair of periods to use to form cross moments, we only consider $T-1$ factors in our model.

So far, our argument has given us the loadings, and $\left\{E \theta_{j}, E \theta_{j}^{2}, E \theta_{j}^{3}, E \varepsilon_{h s, j}^{2}, E \varepsilon_{h s, j}^{3}\right\}_{j=1}^{T-1}$. As a final example consider recovering the fourth power moments. From

$$
E\left(U_{h s, 1}^{3} U_{h s, 2}\right)-3 \alpha_{h s, 2,1} E \theta_{1}^{2} E \varepsilon_{h s, 1}^{2}=\alpha_{h s, 2,1} E \theta_{1}^{4}
$$

for $\theta_{i, 1}$, and from

$$
\left[\begin{array}{c}
E\left(U_{h s, t}^{3} U_{h s, t+1}\right)-\sum_{\ell=1}^{t} \sum_{j=1, j \neq \ell}^{t} 3 \alpha_{h s, t, j}^{2} \alpha_{h s, t, \ell} \alpha_{h s, t+1, \ell} E \theta_{j}^{2} E \theta_{\ell}^{2} \\
-\sum_{j=1}^{t} 3 \alpha_{h s, t, j} \alpha_{h s, t+1, j} E \theta_{j}^{2} E \varepsilon_{h s, t}^{2}-\sum_{j=1}^{t-1} \alpha_{h s, t, j}^{3} \alpha_{h s, t+1, j} E \theta_{j}^{4}
\end{array}\right]=\alpha_{h s, t+1, t} E \theta_{t}^{4}
$$

for $\left\{\theta_{i, j}\right\}_{j=2}^{T-1}$, we can recover all of the fourth order moments of $\theta$. If we form

$$
E \varepsilon_{h s, 1}^{4}=E\left(U_{h s, 1}^{4}\right)-E \theta_{1}^{4}-6 E \theta_{1}^{2} E \varepsilon_{h s, 1}^{2}
$$


for $t=1$; form

$$
E \varepsilon_{h s, t}^{4}=E\left(U_{h s, t}^{4}\right)-\sum_{j=1}^{t-1} \alpha_{h s, t, j}^{4} E \theta_{j}^{4}-E \theta_{t}^{4}-\sum_{j=1}^{t} \sum_{\ell=1 ; j \neq \ell}^{t} 3 \alpha_{h s, t, j}^{2} \alpha_{h s, t, \ell}^{2} E \theta_{j}^{2} E \theta_{\ell}^{2}-\sum_{j=1}^{t} 6 \alpha_{h s, t, j}^{2} E \theta_{j}^{2} E \varepsilon_{h s, t}^{2},
$$

for $t=2, \ldots, T-1$; and form

$$
E \varepsilon_{h s, T}^{4}=E\left(U_{h s, T}^{4}\right)-\sum_{j=1}^{T-1} \alpha_{h s, T, j}^{4} E \theta_{j}^{4}-\sum_{j=1}^{T-1} \sum_{\ell=1 ; j \neq \ell}^{T-1} 3 \alpha_{h s, T, j}^{2} \alpha_{h s, T, \ell}^{2} E \theta_{j}^{2} E \theta_{\ell}^{2}-\sum_{j=1}^{T-1} 6 \alpha_{h s, T, j}^{2} E \theta_{j}^{2} E \varepsilon_{h s, T}^{2},
$$

for $T$, we can then recover $\left\{E \varepsilon_{h s, t}^{4}\right\}_{t=1}^{T}$. It is not hard to see that we can proceeding sequentially with higher moments and in fact recover $\left\{\left\{E \theta_{j}^{k}\right\}_{j=1}^{T-1},\left\{E \varepsilon_{h s, j}^{k}\right\}_{j=1}^{T}\right\}_{k=5}^{\infty}$.

\section{Identifiying loadings - the general case}

In the main text we have shown that we can identify all the loadings when the distributions of the factors are assumed to be nonsymmetric, in particular we showed it using that $\left\{E \theta_{j}^{3} \neq 0\right\}_{j=1}^{T-1}$, but similar arguments could be written with other odd moments. We now show that this assumption is not necessary. For this purpose we add the following assumptions:

(A-4) $(X, K, Z) \Perp(\theta, \varepsilon)$

(A-5) $\theta \Perp \varepsilon_{h s} \Perp \varepsilon_{c o l}$

(A-6) $\theta \Perp \xi, \zeta$

(A-7) $\theta_{1} \Perp \theta_{2} \ldots \Perp \theta_{T-1}$

In what follows, we adopt the following shorthand notation so that we can fit the equations in the page. First, we let $E_{j k} \equiv E\left(U_{h s, j} U_{h s, k}\right), E_{j j j k}=E\left(U_{h s, j}^{3} U_{h s, k}\right)$, and $E_{j k k k}=E\left(U_{h s, j} U_{h s, k}^{3}\right)$ for $1 \leq j, k \leq T$. Second, we drop the $i$ and the $h s$ subscripts, under the understanding that everything we are showing next is for the high school system of wages.

First, we show how to identify $\alpha_{21}$ (i.e., the loading for $\theta_{1}$ at $t=2$ ). Consider the following moments that do not involve odd moments of $\theta_{1}$ or $\theta_{2}$.

$$
\begin{aligned}
E_{11} \equiv E\left(U_{1}^{2}\right) & =E \theta_{1}^{2}+E \varepsilon_{1}^{2} \\
E_{12} \equiv E\left(U_{1} U_{2}\right) & =\alpha_{2,1} E \theta_{1}^{2} \\
E_{22} \equiv E\left(U_{2}^{2}\right) & =\alpha_{2,1}^{2} E \theta_{1}^{2}+E \theta_{2}^{2}+E \varepsilon_{2}^{2} \\
E_{1112} \equiv E\left(U_{1}^{3} U_{2}\right) & =\alpha_{2,1} E \theta_{1}^{4}+3 \alpha_{2,1} E \theta_{1}^{2} E \varepsilon_{1}^{2} \\
E_{1222} \equiv E\left(U_{1} U_{2}^{3}\right) & =\alpha_{2,1}^{3} E \theta_{1}^{4}+3 \alpha_{2,1} E \theta_{1}^{2}\left[E \theta_{2}^{2}+E \varepsilon_{2}^{2}\right] .
\end{aligned}
$$


The first thing to notice is that from $E_{12}$ we know the sign of $\alpha_{21}$. With the sign known, we notice that

$$
\begin{aligned}
E_{1112}-3 E_{12} E_{11} & =\alpha_{2,1} E \theta_{1}^{4}+3 \alpha_{2,1} E \theta_{1}^{2} E \varepsilon_{1}^{2}-3 \alpha_{2,1} E \theta_{1}^{2}\left[E \theta_{1}^{2}+E \varepsilon_{1}^{2}\right] \\
& =\alpha_{2,1}\left[E \theta_{1}^{4}-3 E \theta_{1}^{2} E \theta_{1}^{2}\right]
\end{aligned}
$$

while

$$
\begin{aligned}
E_{1222}-3 E_{12} E_{22} & =\alpha_{2,1}^{3} E \theta_{1}^{4}+3 \alpha_{2,1} E \theta_{1}^{2}\left[E \theta_{2}^{2}+E \varepsilon_{2}^{2}\right]-3 \alpha_{2,1} E \theta_{1}^{2}\left[\alpha_{2,1}^{2} E \theta_{1}^{2}+E \theta_{2}^{2}+E \varepsilon_{2}^{2}\right] \\
& =\alpha_{2,1}^{3} E \theta_{1}^{4}-3 \alpha_{2,1} E \theta_{1}^{2} \alpha_{2,1}^{2} E \theta_{1}^{2} \\
& =\alpha_{2,1}^{3}\left[E \theta_{1}^{4}-3 E \theta_{1}^{2} E \theta_{1}^{2}\right] .
\end{aligned}
$$

It follows then that

$$
\alpha_{2,1}^{2}=\frac{E_{1222}-3 E_{12} E_{22}}{E_{1112}-3 E_{12} E_{11}}
$$

Since we know the sign of $\alpha_{2,1}$ from $E_{12}$, we have identified $\alpha_{2,1}$. Given that, from $E_{12}$ we can recover $E \theta_{1}^{2}$, from $E_{11}$ we get $E \varepsilon_{1}^{2}$, from $E_{22}$ we get $E \theta_{2}^{2}+E \varepsilon_{2}^{2}$, and with all of that, from $E_{1112}$ we get $E \theta_{1}^{4}$. To see how we can identify the remaining loadings related to $\theta_{1}$ simply notice that

$$
E_{1 t}=\alpha_{t, 1} E \theta_{1}^{2}
$$

and hence $\alpha_{t, 1}$ is identified for $t>2$.

We next show how to identify the loadings related to $\theta_{2}$. We define $A_{t k}$ to be $E_{t k}$ minus anything involving only $\theta_{1}$ which is known from our analysis above. Consider thus the following moment functions

$$
\begin{aligned}
A_{22} \equiv E_{22}-\alpha_{2,1}^{2} E \theta_{1}^{2} & =E \theta_{2}^{2}+E \varepsilon_{2}^{2} \\
A_{23} \equiv E_{23}-\alpha_{2,1} \alpha_{3,1} E \theta_{1}^{2} & =\alpha_{3,2} E \theta_{2}^{2} \\
A_{33} \equiv E_{33}-\alpha_{3,1}^{3} E \theta_{1}^{2} & =\alpha_{3,2}^{2} E \theta_{2}^{2}+E \theta_{3}^{2}+E \varepsilon_{3}^{2} \\
A_{2223} \equiv E_{2223}-\alpha_{2,1}^{3} \alpha_{3,1} E \theta_{1}^{4}-3 \alpha_{2,1} E \theta_{1}^{2}\left[\alpha_{3,1} A_{22}+\alpha_{2,1} A_{23}\right] & =\alpha_{3,2} E \theta_{2}^{4}+3 \alpha_{3,2} E \theta_{2}^{2} E \varepsilon_{2}^{2} \\
A_{2333} \equiv E_{2333}-\alpha_{2,1} \alpha_{3,1}^{3} E \theta_{1}^{4}-3 \alpha_{3,1} E \theta_{1}^{2}\left[\alpha_{2,1} A_{33}+\alpha_{3,1} A_{23}\right] & =\alpha_{3,2}^{3} E \theta_{2}^{4}+3 \alpha_{3,2} E \theta_{2}^{2}\left[E \theta_{3}^{2}+E \varepsilon_{3}^{2}\right] .
\end{aligned}
$$

Clearly, this has the exact same structure as the problem above. Hence we can identify $\alpha_{3,2}^{2}=\frac{A_{2333}-3 A_{23} A_{33}}{A_{2223}-3 A_{23} A_{22}}$, and the sign is determined from $A_{23}$. Given that, from $A_{23}$ we can recover $E \theta_{2}^{2}$, from $A_{22}$ we get $E \varepsilon_{2}^{2}$, from $A_{33}$ we get $E \theta_{3}^{2}+E \varepsilon_{3}^{2}$, and with all of that from $A_{2223}$ we get $E \theta_{2}^{4}$. Then from

$$
E_{2 t}-\alpha_{2,1} \alpha_{t, 1} E \theta_{1}^{2}=\alpha_{t, 2} E \theta_{2}^{2}, t>3
$$

we get all of the remaining loadings related to $\theta_{2}$.

We can then proceed sequentially by forming the same set of even moments with the next two equations and recover the loadings for all of the other factors in all of the other periods. There is nothing special about the particular set of moments we picked except that they facilitate the algebra such as it is. Neither 
is the fact that we used second and fourth moments special beyond the simpler algebra they lead to, other even moments could have been used.

\section{Identifying distributions}

Having identified all of the loadings, either by using the arguments in the text or the arguments above, we now proceed to show that the marginal distributions of $\theta$ and $\varepsilon_{h s}$ are nonparametrically identified. As mentioned in the text, in principle we have already provided a way to do this, provided that the distributions of all these random variables can be characterized by their moments. As we show next, this restriction is not required. We first show a theorem that will be useful for this purpose.

Theorem 2. Let $Q_{1}$ and $Q_{2}$ be two random variables that satisfy

$$
\begin{aligned}
& Q_{1}=\theta+R_{1} \\
& Q_{2}=\theta+R_{2}
\end{aligned}
$$

where $\theta, R_{1}$ and $R_{2}$ are mutually independent with $E(\theta)<\infty, E\left(R_{1}\right)=0, E\left(R_{2}\right)=0$, the conditions of Fubini's theorem are satisfied for each random variable and they have non-vanishing (a.e.) characteristic functions. Then, the marginal densities of $\theta, R_{1}$ and $R_{2}$ are identified.

Proof. See Kotlarski (1967), Prakasa Rao (1992).

Using Theorem 2 it is straightforward to show that from

$$
\begin{aligned}
U_{i, h s, 1} & =\theta_{i, 1}+\varepsilon_{i, h s, 1} \\
\frac{U_{i, h s, 2}}{\alpha_{h s, 2,1}} & =\theta_{i, 1}+\left[\frac{\theta_{i, 2}+\varepsilon_{i, h s, 2}}{\alpha_{h s, 2,1}}\right]
\end{aligned}
$$

we can recover $f\left(\theta_{1}\right)$ and $f\left(\varepsilon_{1}\right)$ (as well as $f\left(\theta_{2}+\varepsilon_{2}\right)$ ). Now consider the next pair of equations

$$
\begin{aligned}
U_{i, h s, 2} & =\theta_{i, 2}+\left[\theta_{i, 1} \alpha_{h s, 2,1}+\varepsilon_{i, h s, 2}\right]=\theta_{i, 2}+Z_{i, 2} \\
\frac{U_{i, h s, 3}}{\alpha_{h s, 3,2}} & =\theta_{i, 2}+\left[\frac{\theta_{i, 1} \alpha_{h s, 3,1}+\varepsilon_{i, h s, 3}}{\alpha_{h s, 3,2}}\right]=\theta_{i, 2}+Z_{i, 3 .} .
\end{aligned}
$$

Since $\theta_{i, 1}$ enters both terms on brackets in each equation, the assumptions of Theorem 2 are not satisfied as the three random variables, $\theta_{i, 2}, Z_{i, 2}, Z_{i, 3}$ are not mutually independent. The following Theorem shows that, even though this is the case, the fact that the source of the dependence is $\theta_{i, 1}$ and we know its distribution from before (hence its characteristic function) is enough to provide identification.

Theorem 3. Let $Q_{1}$ and $Q_{2}$ be two random variables that satisfy

$$
\begin{aligned}
& Q_{1}=\theta+R_{1}=\theta+\eta a_{1}+e_{1} \\
& Q_{2}=\theta+R_{2}=\theta+\eta a_{2}+e_{2}
\end{aligned}
$$


where $a_{1}, a_{2}$ are known constants, $\theta, \eta, e_{1}$ and $e_{2}$ are mutually independent with $E \theta<\infty, E \eta=E e_{1}=$ $E e_{2}=0$, the conditions of Fubini's theorem are satisfied for each random variable and they have nonvanishing (a.e.) characteristic functions. Furthermore, let the characteristic function of $\eta$ be denoted by $\phi_{\eta}(t)$ and assume it is known. Then, the marginal densities of $\theta, e_{1}$ and $e_{2}$ are identified.

Proof. To prove this theorem we follow the proof of Theorem 2 very closely. Let $\phi_{Q_{1}, Q_{2}}\left(t_{1}, t_{2}\right)$ be the characteristic function of $Q_{1}, Q_{2}$, and define the rest of the characteristic functions equivalently. We have

$$
\begin{aligned}
\phi_{Q_{1}, Q_{2}}\left(t_{1}, t_{2}\right) & =E\left[e^{i\left(t_{1} Q_{1}+t_{2} Q_{2}\right)}\right] \\
& =E\left[e^{i\left(t_{1}\left(\theta+R_{1}\right)+t_{2}\left(\theta+R_{2}\right)\right)}\right] \\
& =E\left[e^{i\left(\left(t_{1}+t_{2}\right) \theta+t_{1} R_{1}+t_{2} R_{2}\right)}\right] \\
& =E\left[e^{i\left(t_{1}+t_{2}\right) \theta} e^{i\left(t_{1} R_{1}+t_{2} R_{2}\right)}\right] \\
& =E\left[e^{i\left(t_{1}+t_{2}\right) \theta}\right] E\left[e^{i\left(t_{1} R_{1}+t_{2} R_{2}\right)}\right] \\
& =\phi_{\theta}\left(t_{1}+t_{2}\right) \phi_{R_{1}, R_{2}}\left(t_{1}, t_{2}\right),
\end{aligned}
$$

where the last two equalities follow from the independence between $\theta$ and $\left(R_{1}, R_{2}\right)$. Now let's look at $\phi_{R_{1}, R_{2}}\left(t_{1}, t_{2}\right)$ in more detail.

$$
\begin{aligned}
\phi_{R_{1}, R_{2}}\left(t_{1}, t_{2}\right) & =E\left[e^{i\left(t_{1}\left(\eta a_{1}+e_{1}\right)+t_{2}\left(\eta a_{2}+e_{2}\right)\right)}\right] \\
& =E\left[e^{i\left(\left(t_{1} a_{1}+t_{2} a_{2}\right) \eta+t_{1} e_{1}+t_{2} e_{2}\right)}\right] \\
& =\phi_{e_{1}}\left(t_{1}\right) \phi_{e_{2}}\left(t_{2}\right) \phi_{\eta}\left(t_{1} a_{1}+t_{2} a_{2}\right) .
\end{aligned}
$$

Replace into $\phi_{Q_{1}, Q_{2}}\left(t_{1}, t_{2}\right)$ to get

$$
\phi_{Q_{1}, Q_{2}}\left(t_{1}, t_{2}\right)=\left[\phi_{\theta}\left(t_{1}+t_{2}\right) \phi_{e_{1}}\left(t_{1}\right) \phi_{e_{2}}\left(t_{2}\right)\right] \phi_{\eta}\left(t_{1} a_{1}+t_{2} a_{2}\right) .
$$

Continuing along the lines of the proof in Kotlarski (1967), we now define $\tilde{\theta}, \tilde{\eta}, \tilde{e}_{1}, \tilde{e}_{2}$ be four new random variables that are also independent of each other. Let

$$
\begin{gathered}
V_{1}=\tilde{\theta}+\tilde{\eta} a_{1}+\tilde{e}_{1} \\
V_{2}=\tilde{\theta}+\tilde{\eta} a_{2}+\tilde{e}_{2},
\end{gathered}
$$

where $a_{1}$ and $a_{2}$ are the same known constants as before. We let $\psi$ denote the respective characteristic functions of these new random variables. Following the same line of arguments as above we get

$$
\psi_{V_{1}, V_{2}}\left(t_{1}, t_{2}\right)=\left[\psi_{\tilde{\theta}}\left(t_{1}+t_{2}\right) \psi_{\tilde{e}_{1}}\left(t_{1}\right) \psi_{\tilde{e}_{2}}\left(t_{2}\right)\right] \psi_{\tilde{\eta}}\left(t_{1} a_{1}+t_{2} a_{2}\right)
$$

The key to our results is to notice that we can set

$$
\phi_{\eta}\left(t_{1} a_{1}+t_{2} a_{2}\right)=\psi_{\tilde{\eta}}\left(t_{1} a_{1}+t_{2} a_{2}\right),
$$


since $\phi_{\eta}(t)$ is known from our previous analysis.

As in Kotlarski (1967) we now assume that the pairs $Q_{1}, Q_{2}$ and $V_{1}, V_{2}$ have the same distribution. We thus have

$$
\phi_{\theta}\left(t_{1}+t_{2}\right) \phi_{e_{1}}\left(t_{1}\right) \phi_{e_{2}}\left(t_{2}\right) \phi_{\eta}\left(t_{1} a_{1}+t_{2} a_{2}\right)=\left[\psi_{\tilde{\theta}}\left(t_{1}+t_{2}\right) \psi_{\tilde{e}_{1}}\left(t_{1}\right) \psi_{\tilde{e}_{2}}\left(t_{2}\right)\right] \psi_{\tilde{\eta}}\left(t_{1} a_{1}+t_{2} a_{2}\right)
$$

From (26) we can simplify to get

$$
\phi_{\theta}\left(t_{1}+t_{2}\right) \phi_{e_{1}}\left(t_{1}\right) \phi_{e_{2}}\left(t_{2}\right)=\psi_{\tilde{\theta}}\left(t_{1}+t_{2}\right) \psi_{\tilde{e}_{1}}\left(t_{1}\right) \psi_{\tilde{e}_{2}}\left(t_{2}\right)
$$

Since equation (27) is identical to equation (9) in Kotlarski (1967), the results follows directly from the rest of the proof in Kotlarski (1967).

Clearly, the system given by

$$
\begin{aligned}
U_{i, h s, 2} & =\theta_{i, 2}+\left[\theta_{i, 1} \alpha_{h s, 2,1}+\varepsilon_{i, h s, 2}\right] \\
\frac{U_{i, h s, 3}}{\alpha_{h s, 3,2}} & =\theta_{i, 2}+\left[\theta_{i, 1} \frac{\alpha_{h s, 3,1}}{\alpha_{h s, 3,2}}+\frac{\varepsilon_{i, h s, 3}}{\alpha_{h s, 3,2}}\right]
\end{aligned}
$$

fits exactly the conditions of Theorem (3) and hence $f\left(\theta_{2}\right), f\left(\varepsilon_{h s, 2}\right)$ and $f\left(\varepsilon_{h s, 3}\right)$ are identified. By proceeding sequentially we can identify all of the remaining distributions of the high school wages system

From our analysis above, we now have knowledge of the nonparametric distribution of the factors and uniquenesses as well as the loadings for wages for $s=h s$. We next turn our attention to identification to the analogous system of equations in $(25)$ for college $(s=c o l)$.

Corollary 1. Under $(\boldsymbol{A}-\mathbf{1})$ - $(\boldsymbol{A}-\boldsymbol{7})$, both the loadings $\alpha_{c o l, t}$ and the nonparametric distribution of $\varepsilon_{c o l, t}$, $t=1, \ldots T$, are identified without further normalizations.

Proof. To see why, take $U_{c o l, 1}$ and an arbitrarily chosen period $t>1$. Now form

$$
\frac{E\left(U_{c o l, 1}^{2} U_{c o l, t}\right)}{E\left(U_{c o l, 1} U_{c o l, t}\right)}=\alpha_{c o l, 1,1} \frac{E\left(\theta_{1}^{3}\right)}{E\left(\theta_{1}^{2}\right)} .
$$

Since we know the distribution of $\theta_{1}$ from our analysis of the high school system, we know $\frac{E\left(\theta_{1}^{3}\right)}{E\left(\theta_{1}^{2}\right)}$ and hence we can recover $\alpha_{c o l, 1,1}$. Now, by forming

$$
\operatorname{cov}\left(U_{c o l, 1} U_{c o l, t}\right)=\alpha_{c o l, 1,1} \alpha_{c o l, t, 1} E\left(\theta_{1}^{2}\right)
$$

we can recover $\alpha_{c o l, t, 1}$. Since $t$ was chosen arbitrarily, we can recover the loadings for factor 1 for all periods. The nonparametric distribution of $\varepsilon_{c o l, 1}$ can be recovered from $U_{c o l, 1}=\theta_{1} \alpha_{c o l, 1,1}+\varepsilon_{c o l, 1}$ by deconvolution. Proceeding sequentially, the loadings on the remaining factors as well as the nonparametric distributions of the remaining uniquenesses can also be recovered. 


\section{Identification of the Cost Function}

Identification of the cost function follows from the arguments in Heckman and Navarro (2007) which we repeat here for completeness. Write the college attendance condition (7) as

$$
E\left(\mathscr{V}_{c o l, 1}\left(\mathcal{I}_{1}\right)-\mathscr{V}_{h s, 1}\left(\mathcal{I}_{1}\right)-\phi(Z)-\theta \lambda-\omega \mid \mathcal{I}_{0}\right)>0
$$

Let $\theta^{0}$ denote the elements of $\theta$ included in the agent's information set at the time the schooling decision is made, and let $\lambda^{0}$ denote the sub-vector of $\lambda$ associated with them. Define $E\left(\mathscr{V}_{\text {col, } 1}^{*}\left(\mathcal{I}_{1}\right)-\mathscr{V}_{h s, 1}^{*}\left(\mathcal{I}_{1}\right) \mid \mathcal{I}_{0}\right)=$ $\mu_{V}(X)+\tau\left(X, \theta^{0}\right)$ which is known since all of the elements are known from our previous analysis. The econometrician has data on the left hand side of

$$
\operatorname{Pr}(S=\operatorname{col} \mid X, Z)=\operatorname{Pr}\left(\tau\left(X, \theta^{0}\right)-\theta^{0} \lambda^{0}-\omega>\phi(Z)-\mu_{V}(X)\right)
$$

Theorem 4. Assume that the relevant elements of (A-1) hold. Change (A-2) so that the independence of $Z$ and the error terms holds conditional on $X$. Let $Z^{e}$ be the elements of $Z$ that are not a part of $X$ (excluded from wages) and further assume that we can define $\phi\left(z^{e}, x\right)$ for all pairs $\left(z^{e}, x\right)$ in the support of $Z$. As with all discrete choice problems the scale needs to be set. Assume that

(A-8) $\operatorname{var}\left(\tau\left(\widetilde{x}, \theta^{0}\right)-\theta^{0} \lambda^{0}-\omega\right)=1$ for $X=\widetilde{x}$.

Then, if $\phi(Z)$ satisfies the is part of the Matzkin class of functions (Matzkin, 1992; Heckman and Navarro, 2007), $\lambda^{0}$ and the nonparametric distribution of $\omega$ are identified up to normalization.

Proof. Define $\Upsilon\left(X, \theta^{0}\right)=\tau\left(X, \theta^{0}\right)-\theta^{0} \lambda^{0}-\omega$ and fix $X=\widetilde{x}$. The observed probability that the agent chooses college (conditional on $X=\widetilde{x}$ and using A-3) is $\operatorname{Pr}\left(\Upsilon\left(\widetilde{x}, \theta^{0}\right)>\phi\left(Z^{e}, \widetilde{x}\right)-\mu_{V}(\widetilde{x})\right)$ where $\mu_{V}(\widetilde{x})$ is a known constant (conditional on $X=\widetilde{x}$ ). Using the (conditional on $X$ ) independence of $\theta^{0} \lambda_{0}+\omega$ and $Z^{e}$ we can then use the analysis of Matzkin (1992) to identify $\phi\left(Z^{e}, \widetilde{x}\right)$ and the distribution of $\Upsilon\left(\widetilde{x}, \theta^{0}\right)$, all of this conditional on $X=\widetilde{x}$, up to a normalization.

Next, still conditional on $X=\widetilde{x}$, we can form the joint distribution of log wages and the choice index since we know the left hand side of

$$
\begin{gathered}
\operatorname{Pr}\left(U_{s} \leq \ln W_{s}-\mu_{s}(\widetilde{x}) \mid X=\widetilde{x}, S=s, Z^{e}=z^{e}\right) \operatorname{Pr}\left(S=s \mid Z^{e}=z^{e}\right) \\
=\int_{-\infty}^{\ln W_{s}-\mu_{s}(\widetilde{x})} \int_{\phi\left(z^{e}, \widetilde{x}\right)-\mu_{V}(\widetilde{x})}^{\infty} f\left(U_{s}, \Upsilon\left(\widetilde{x}, \theta^{0}\right)\right) d \Upsilon\left(\widetilde{x}, \theta^{0}\right) d U_{s} .
\end{gathered}
$$

We can trace this integral by varying $\ln W_{s}, z_{E}$, so we can identify the joint distribution of $U_{s}, \Upsilon\left(X, \theta^{0}\right)$. With the joint distribution in hand, we can follow the reasoning of theorem 1 to identify $\lambda^{0}$. To see how, take the covariance between the choice index and the first equation for high school :

$$
\operatorname{cov}\left(\Upsilon\left(\widetilde{x}, \theta^{0}\right), U_{h, 1}\right)=\operatorname{cov}\left(\tau\left(\widetilde{x}, \theta^{0}\right), \theta_{1}\right)+\lambda_{1}^{0} \sigma_{\theta_{1}}^{2}
$$

where everything but $\lambda_{1}^{0}$ is known so we can solve for it. Proceeding recursively we identify all of the elements of $\lambda^{0}$. 
With $\lambda^{0}$ known, we can take $\Upsilon\left(\widetilde{x}, \theta^{0}\right)$ and deconvolve the distribution of $\omega$ since it is independent of $X, \theta$ by A-2 and the factor structure assumptions. Finally, by changing the value of $z^{e}, \widetilde{x}$ we can trace $\phi\left(Z^{e}, X\right)$ coordinate by coordinate since, for any value of $X$, the scale $\operatorname{var}\left(\tau\left(X, \theta^{0}\right)-\theta^{0} \lambda_{0}-\omega\right)$ is a function of known elements. 


\section{Appendix 2: A General Procedure}

We cast the problem of determining agent information sets as a testing problem. ${ }^{51}$ We develop a simple test of misspecification for a proposed information set that does not depend on the details of the particular model being used. In this section we deal with the test of whether a candidate information set is correctly specified in a general setting. ${ }^{52}$

Let $\pi_{\tau, t}^{H}$ and $\pi_{\tau, t}^{C}$ be a set of auxiliary parameters. To define the proposed test, instead of basing the likelihood on equations (15) and (16) in the text, we use

$$
\begin{aligned}
& \ln \widehat{H}_{i, t}=\mathscr{G}_{s, t}^{H}\left(\widetilde{\mathcal{I}}_{i, t}\right)+\delta_{H}^{\prime} K_{i, t}^{H}+\xi_{i, t}^{H}+\sum_{\tau=t+1}^{T}\left[Y_{i, s, \tau}-E\left(Y_{i, s, \tau} \mid \widetilde{\mathcal{I}}_{i, t}\right)\right] \pi_{\tau, t}^{H}, \\
& \ln \widehat{C}_{i, t}=\mathscr{G}_{s, t}^{C}\left(\widetilde{\mathcal{I}}_{i, t}\right)+\delta_{C}^{\prime} K_{i, t}^{C}+\xi_{i, t}^{C}+\sum_{\tau=t+1}^{T}\left[Y_{i, s, \tau}-E\left(Y_{i, s, \tau} \mid \widetilde{\mathcal{I}}_{i, t}\right)\right] \pi_{\tau, t}^{C} .
\end{aligned}
$$

By assumption, the predicted hours and consumption $\left(\mathscr{G}_{s, t}^{H}\left(\widetilde{\mathcal{I}}_{i, t}\right), \mathscr{G}_{s, t}^{C}\left(\widetilde{\mathcal{I}}_{i, t}\right)\right)$ will not depend on the earnings innovations included in the last term on the right hand side of each equation since the agent integrates them out. The actual decisions, however, will be a function of the true agent's information set at $t$ which may contain elements of $\left\{Y_{i, s, \tau}-E\left(Y_{i, s, \tau} \mid \widetilde{\mathcal{I}}_{i, t}\right)\right\}_{\tau=t+1}^{T}$. A test of which of the auxiliary parameters multiplying the earnings innovations $\left\{\pi_{\tau, t}^{H}, \pi_{\tau, t}^{C}\right\}_{\tau=t+1}^{T}$ equal zero is then a test of whether the proposed agent's information set at time $t$ is correctly specified. ${ }^{53}$ Notice that, as done in Cunha et al., 2005, the schooling choice (or any other choice) may also be used when testing.

\footnotetext{
${ }^{51}$ See Cunha et al., 2005 and Cunha and Heckman (2016) where a version of this test for a perfect credit markets model of schooling choice is proposed.

${ }^{52}$ The particular implementation of the test used in this paper is shown in section 3.2.

${ }^{53}$ And it can in fact be considered as a form of Sims' test of causality (Sims, 1972).
} 
Figure 1

Ability $(Q)$

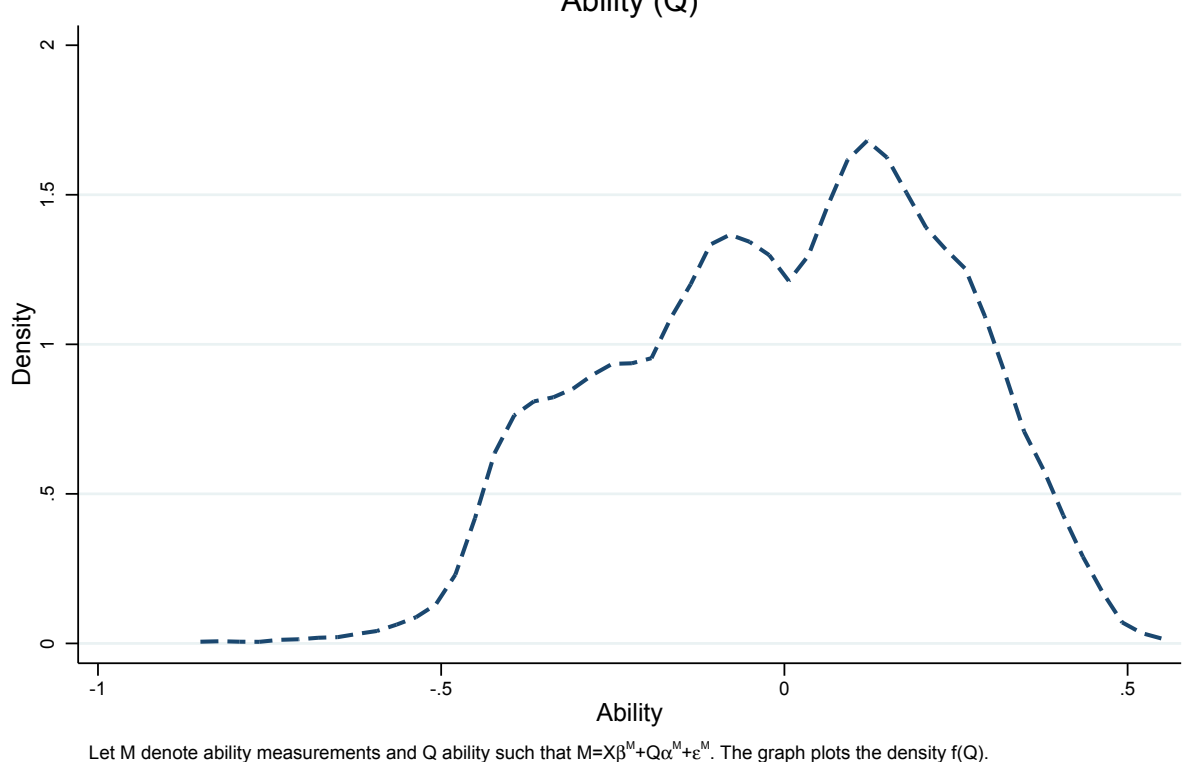

Figure 2.1

Densities of Ability (Q) Conditional on Schooling Choice

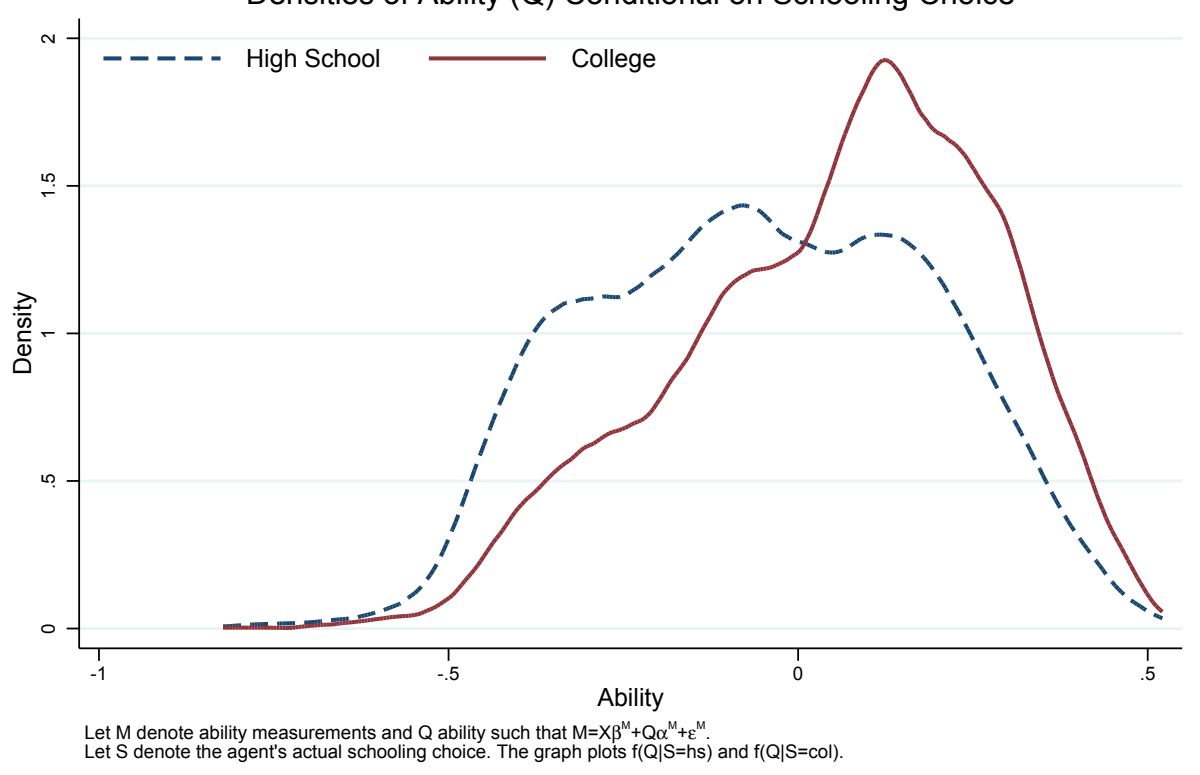


Figure 2.2

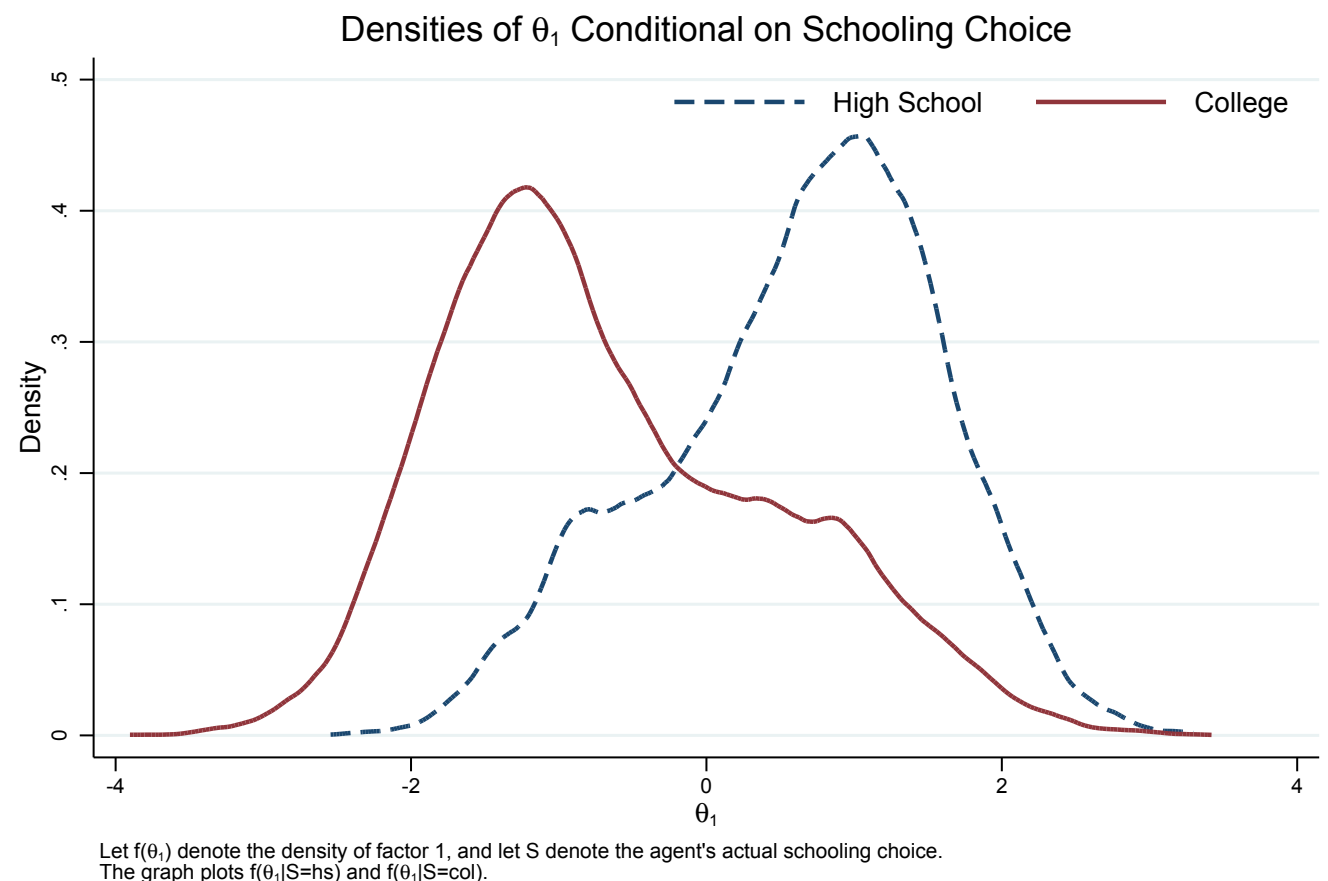

Figure 2.3

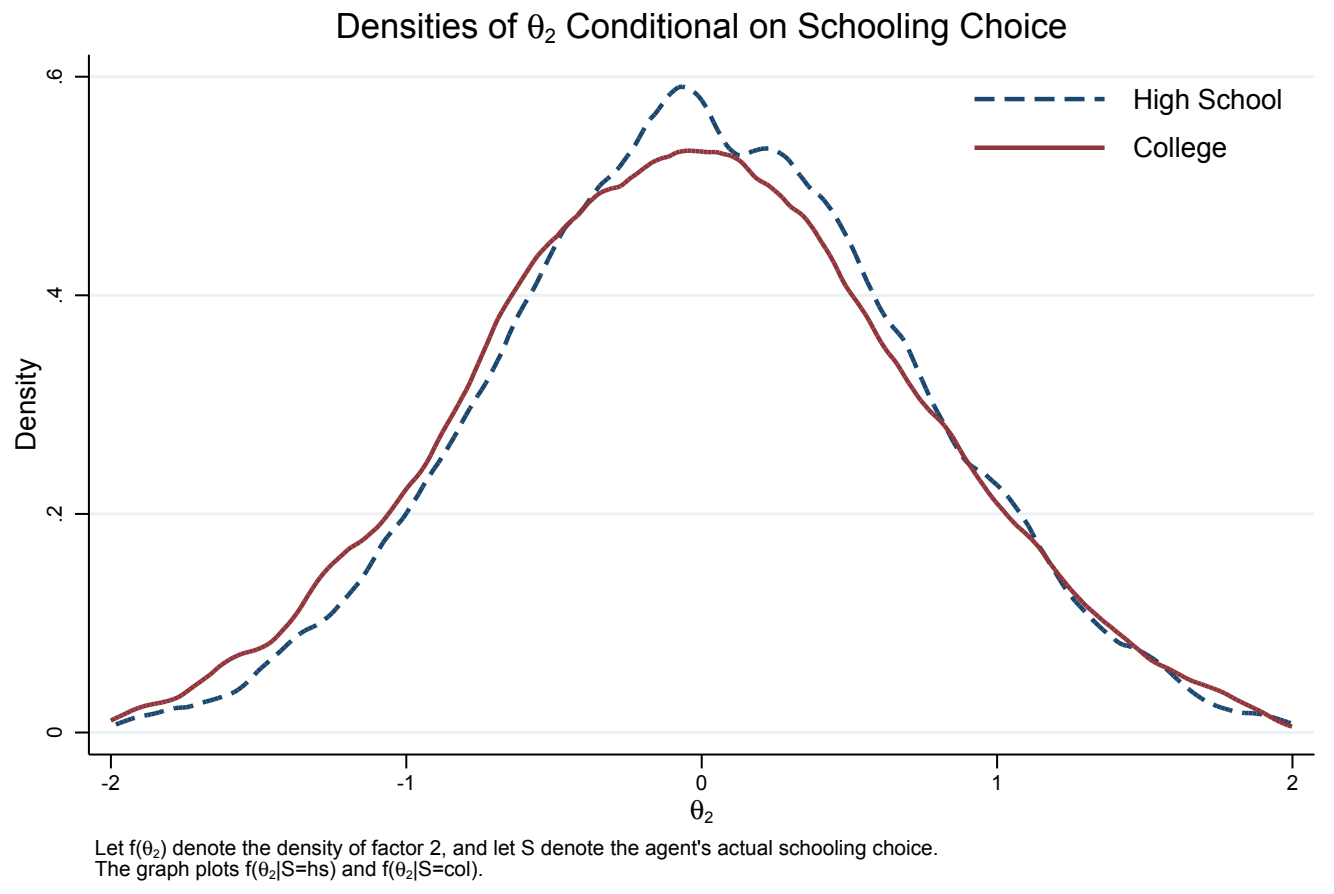


Figure 2.4

\section{Densities of $\theta_{7}$ Conditional on Schooling Choice}

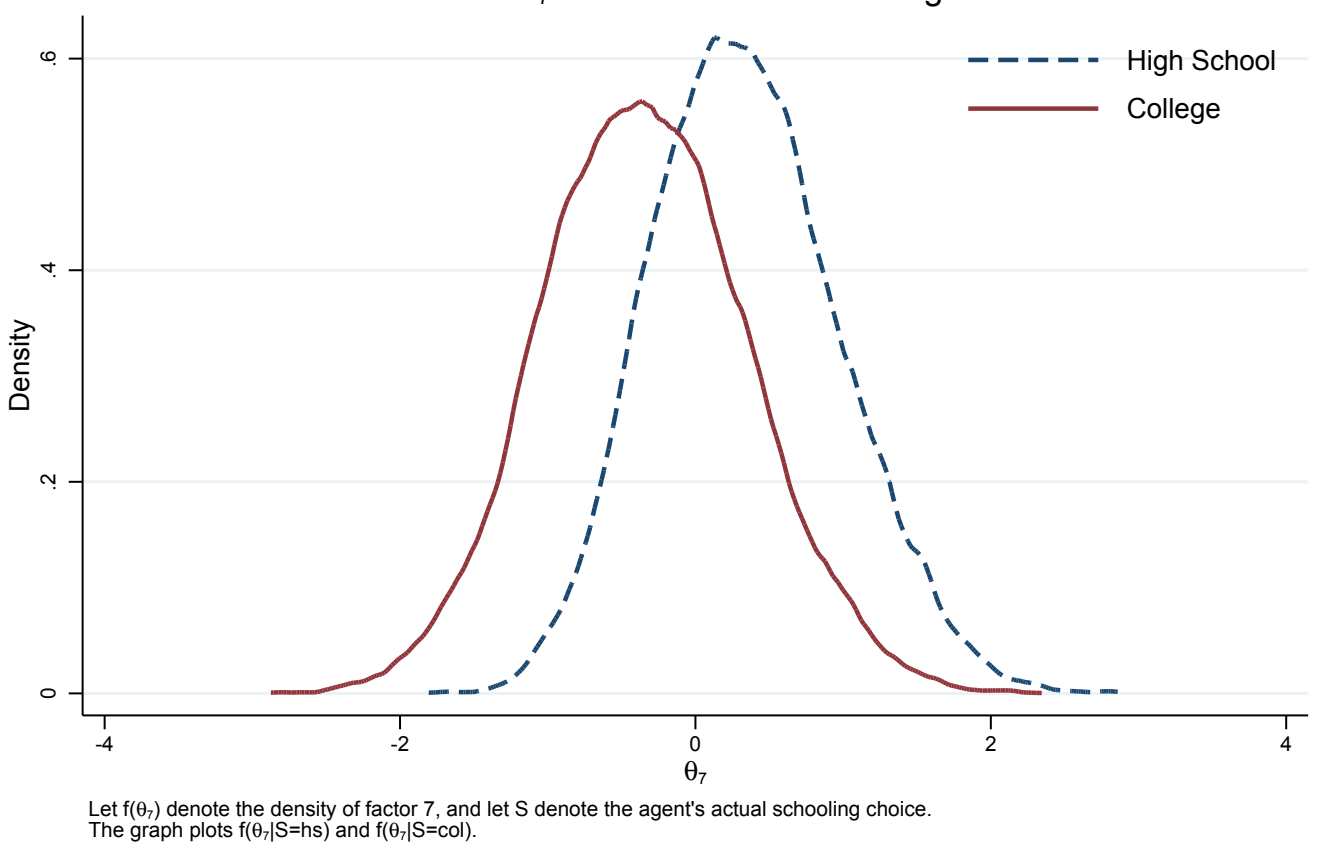

Figure 3.1

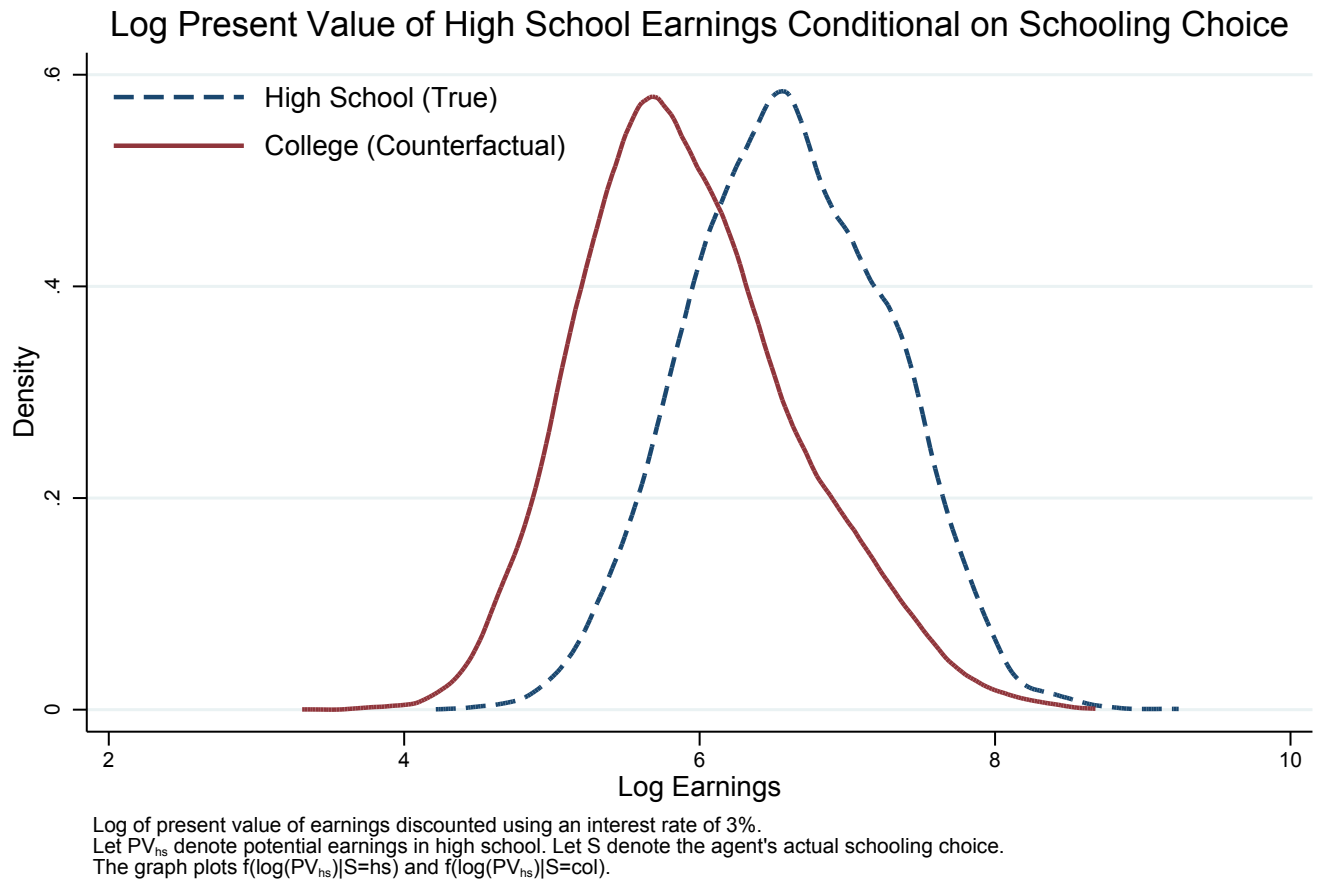


Figure 3.2

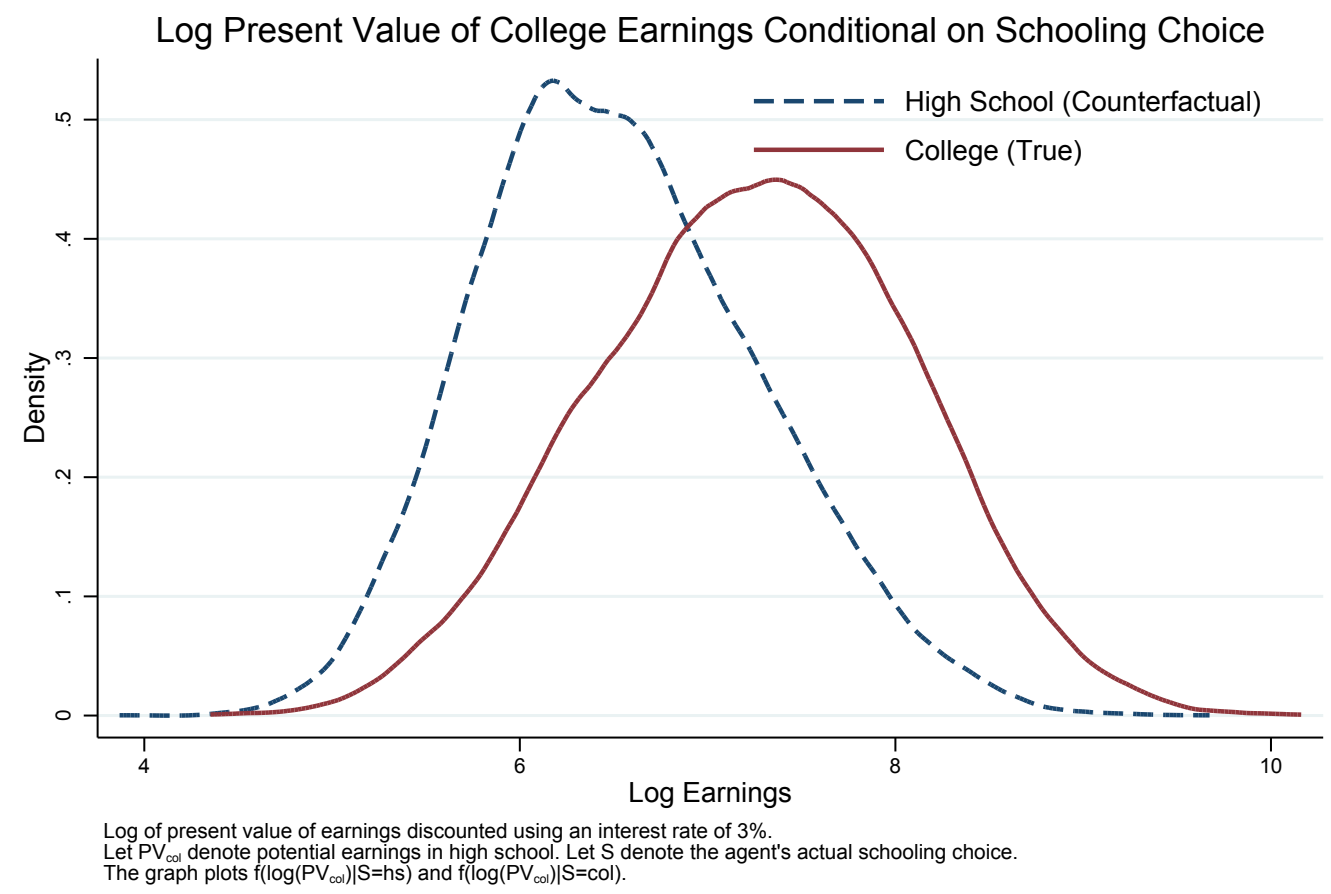

Figure 3.3

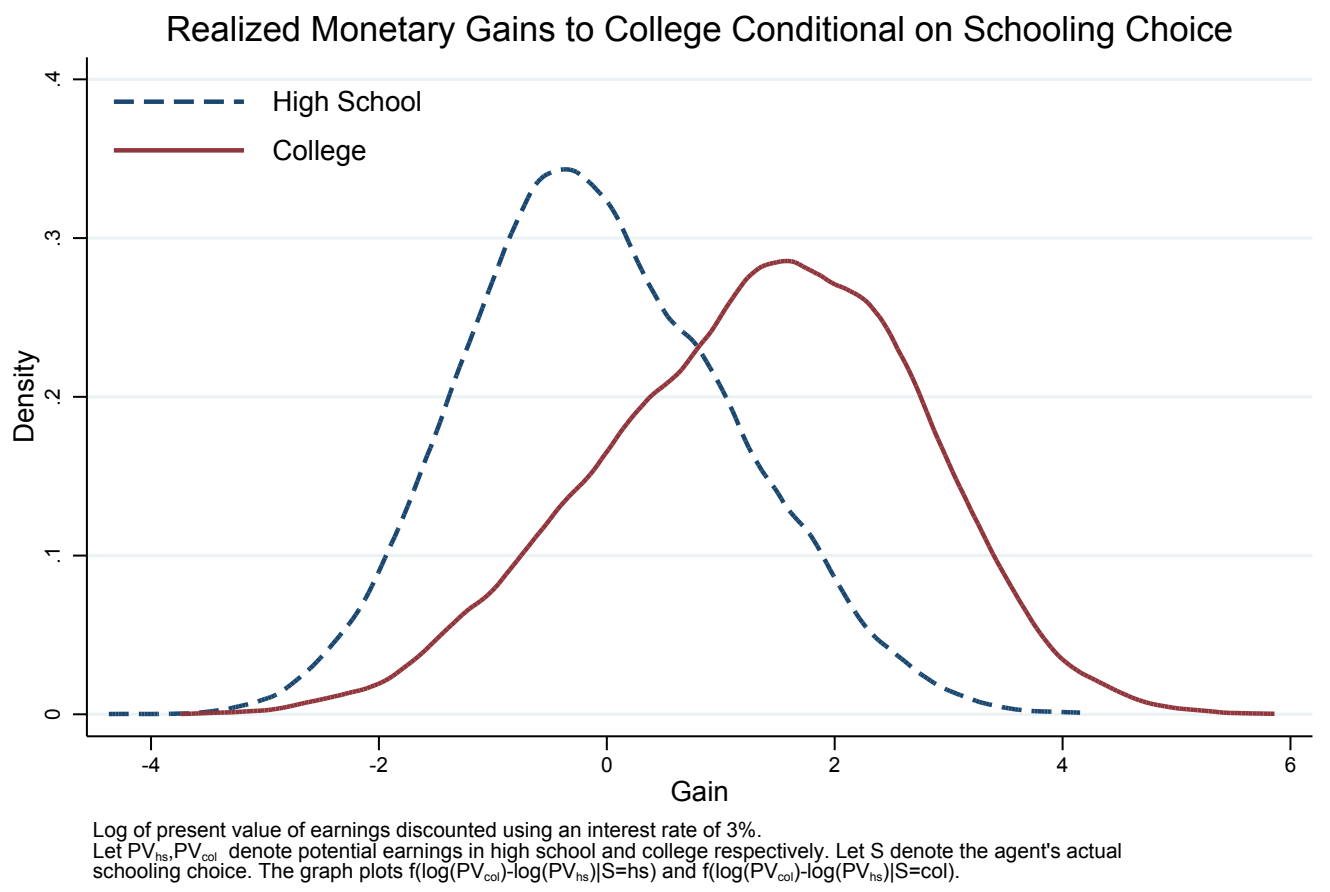


Figure 4.1

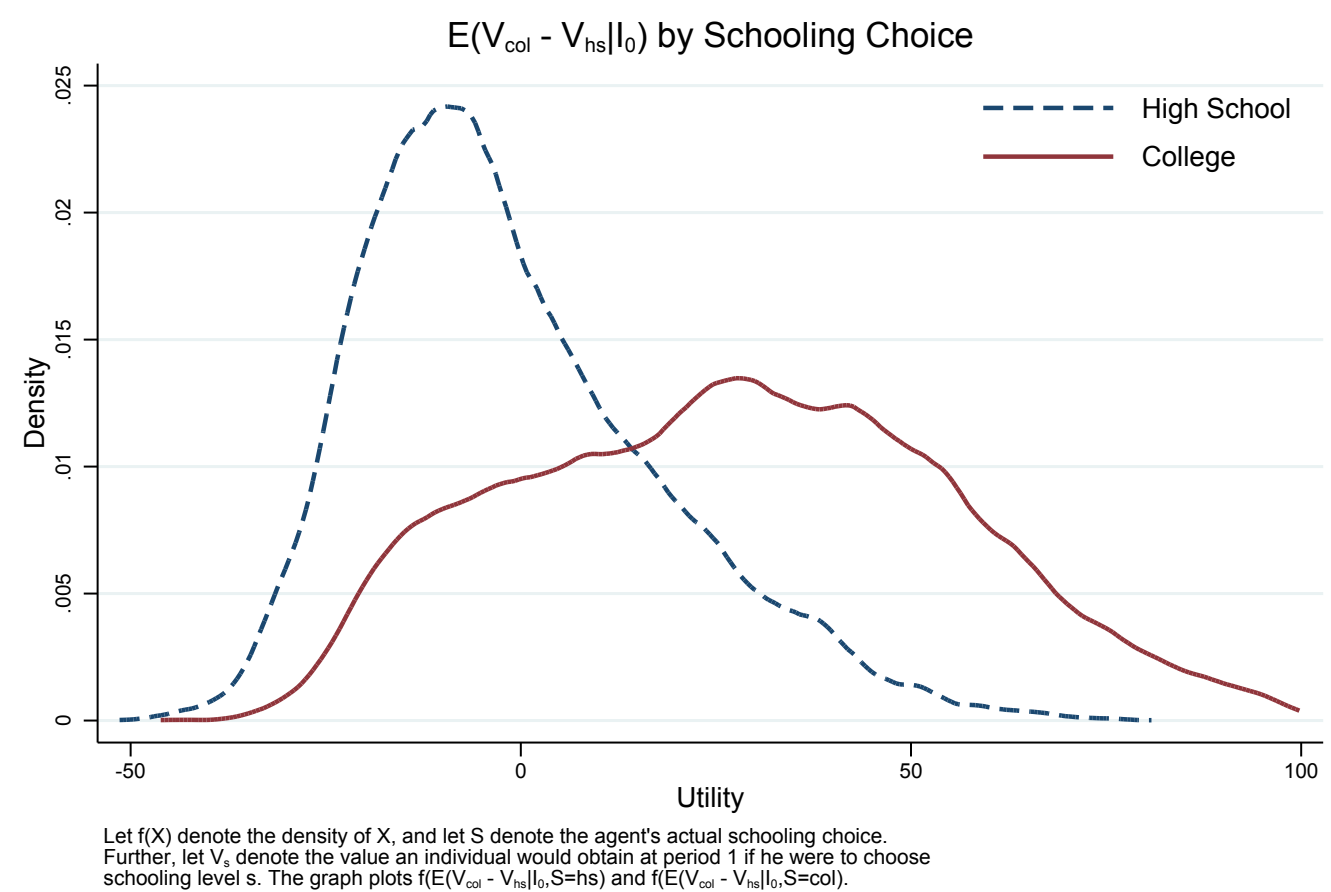

Figure 4.2

Psychic Cost of College Conditional on Schooling Choice

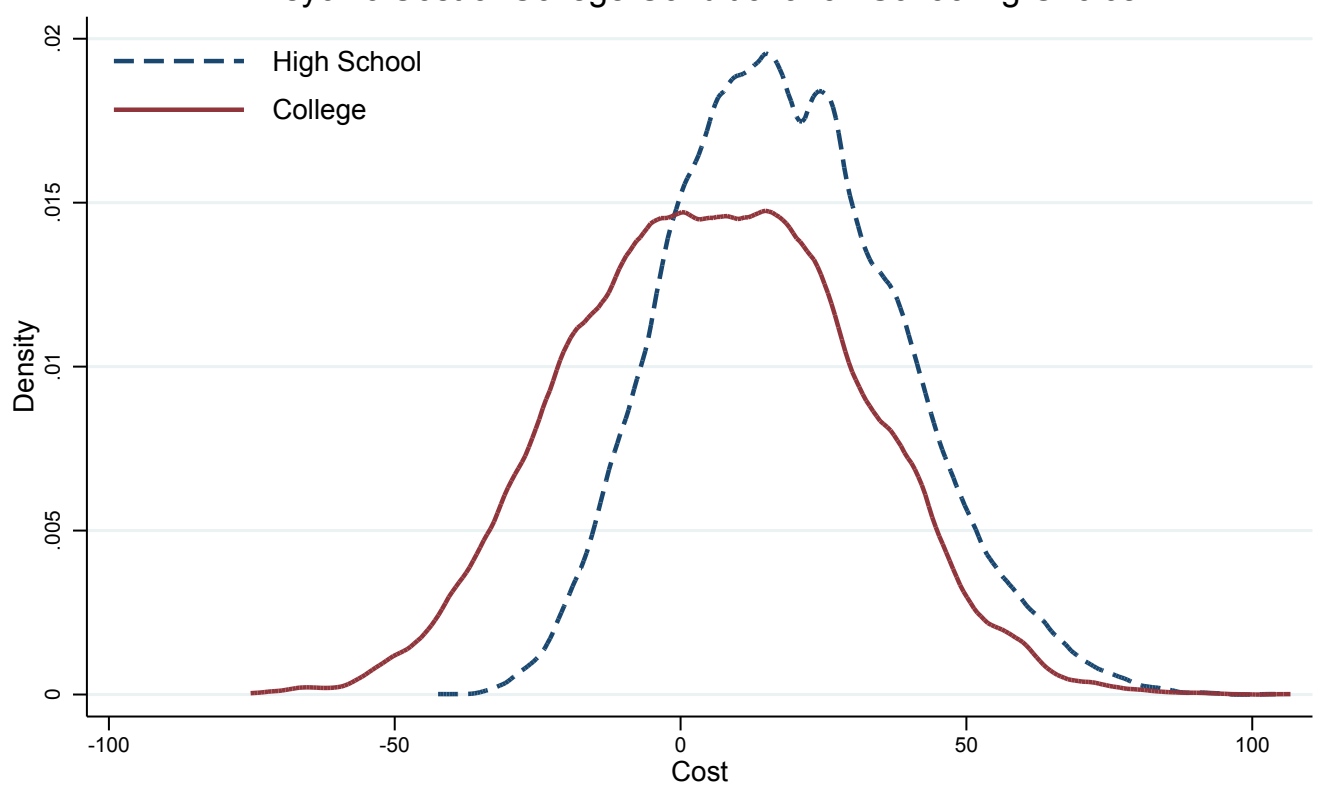

Let $f(P)$ denote the density of Psychic costs, and let $S$ denote the individuals schooling choice. The graph plots $f(P \mid S=h s)$ and $f(P \mid S=c o l)$. 


\section{Figure 5: Factor Distribution for Insurance Switchers}

Moving from Asymmetric Information to Equal Information Scenario

(a) Distribution of $\theta_{1}$

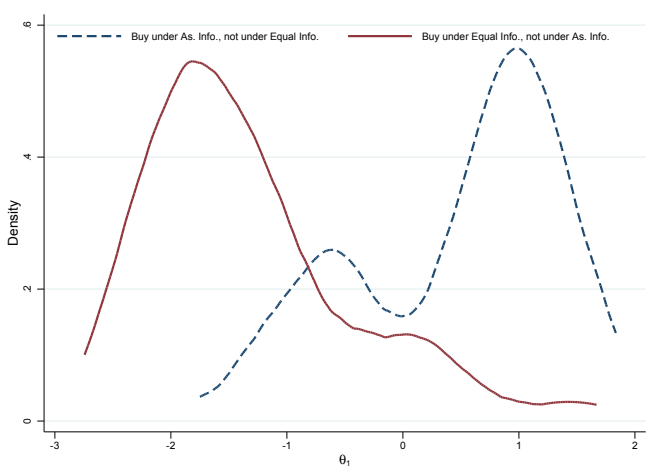

(b) Distribution of $\theta_{2}$

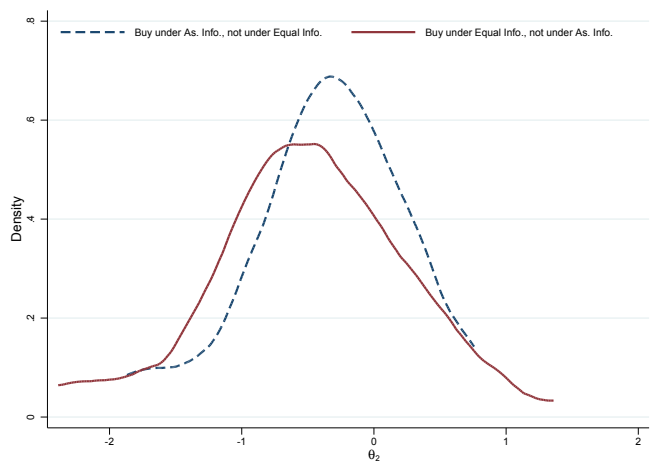

(c) Distribution of $\theta_{7}$

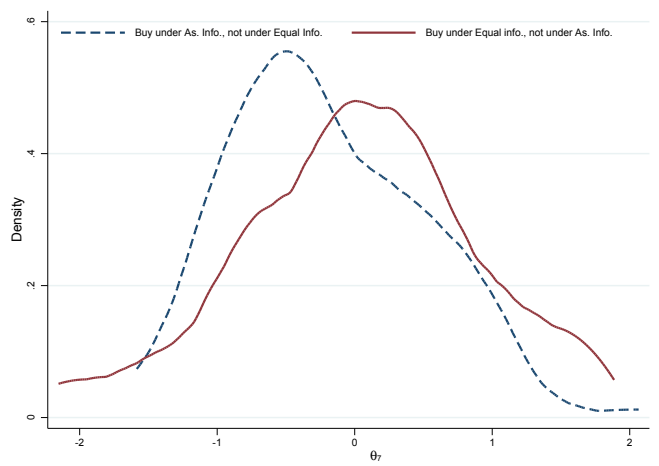

1. Buy under As. Info., not under Equal Info.: people who buy insurance in the asymmetric information case but not in the equal information one.

2. Buy under Equal info., not under As. Info.: people who don't buy insurance under asymmetric information but do buy it under equal information.

3. Under scenario: Asymmetric Information, the bank does not know any of the factors at $\mathrm{t}=0$, but the agent has access to $\theta_{1}, \theta_{2}$, and $\theta_{7}$

4. Under scenario: Equal Information, the bank and the agent have the same information at $\mathrm{t}=0$. The information set now includes $\theta_{1}$, $\theta_{2}$, and $\theta_{7}$. 
Figure 6: The Distribution of Premium Differences $\left(m^{E}-m^{A}\right)$ for those who drop insurance when adverse selection is removed

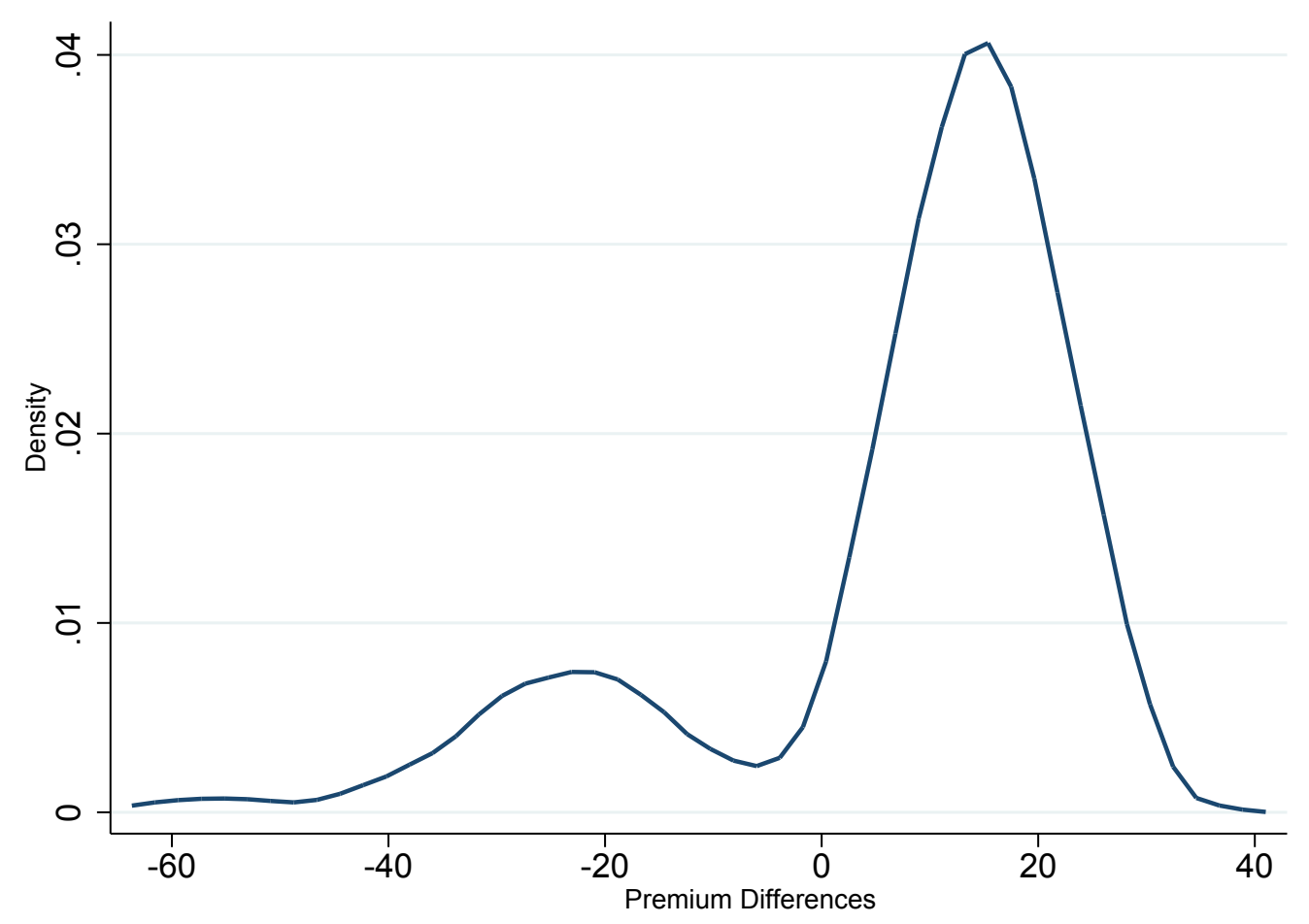

1. Let $m^{A}$ denote the premium charged to an individual in the asymmetric information scenario. Let $m^{E}$ denote the premium charged to an individual in the equal information scenario.

2. Under scenario: Asymmetric Information, the bank does not know any of the factors at $\mathrm{t}=0$, but the agent has access to $\theta_{1}$, $\theta_{2}$, and

3. Under scenario: Equal Information, the bank and the agent have the same information at $\mathrm{t}=0$. The information set now includes $\theta_{1}$,

3. Under sce 
Figure 7: The Distribution of Consumer Lifecycle Welfare

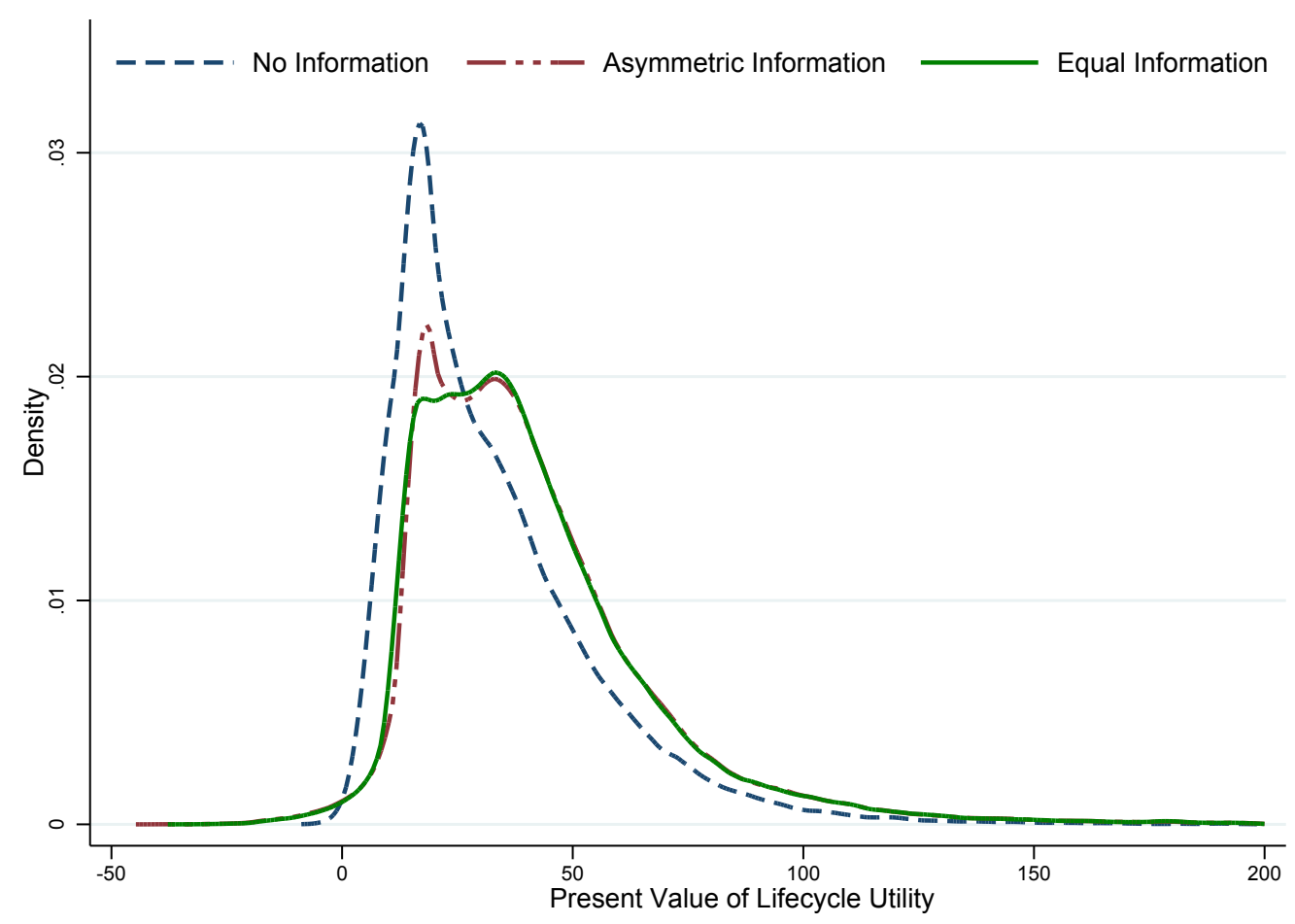

1. Under scenario: No Information, the bank and the agent have the same information at $\mathrm{t}=0$. The information set does not include $\theta$. 2. Under scenario: Asymmetric Information, the bank does not know any of the factors at $\mathrm{t}=0$, but the agent has access to $\theta_{1}$, $\theta_{2}$, and

$\theta_{7}$.

3. Under scenario: Equal Information, the bank and the agent have the same information at $\mathrm{t}=0$. The information set now includes $\theta_{1}$,
$\theta_{2}$, and $\theta_{7}$. 
Figure 8: The Distribution of Present Value of Bank Profits

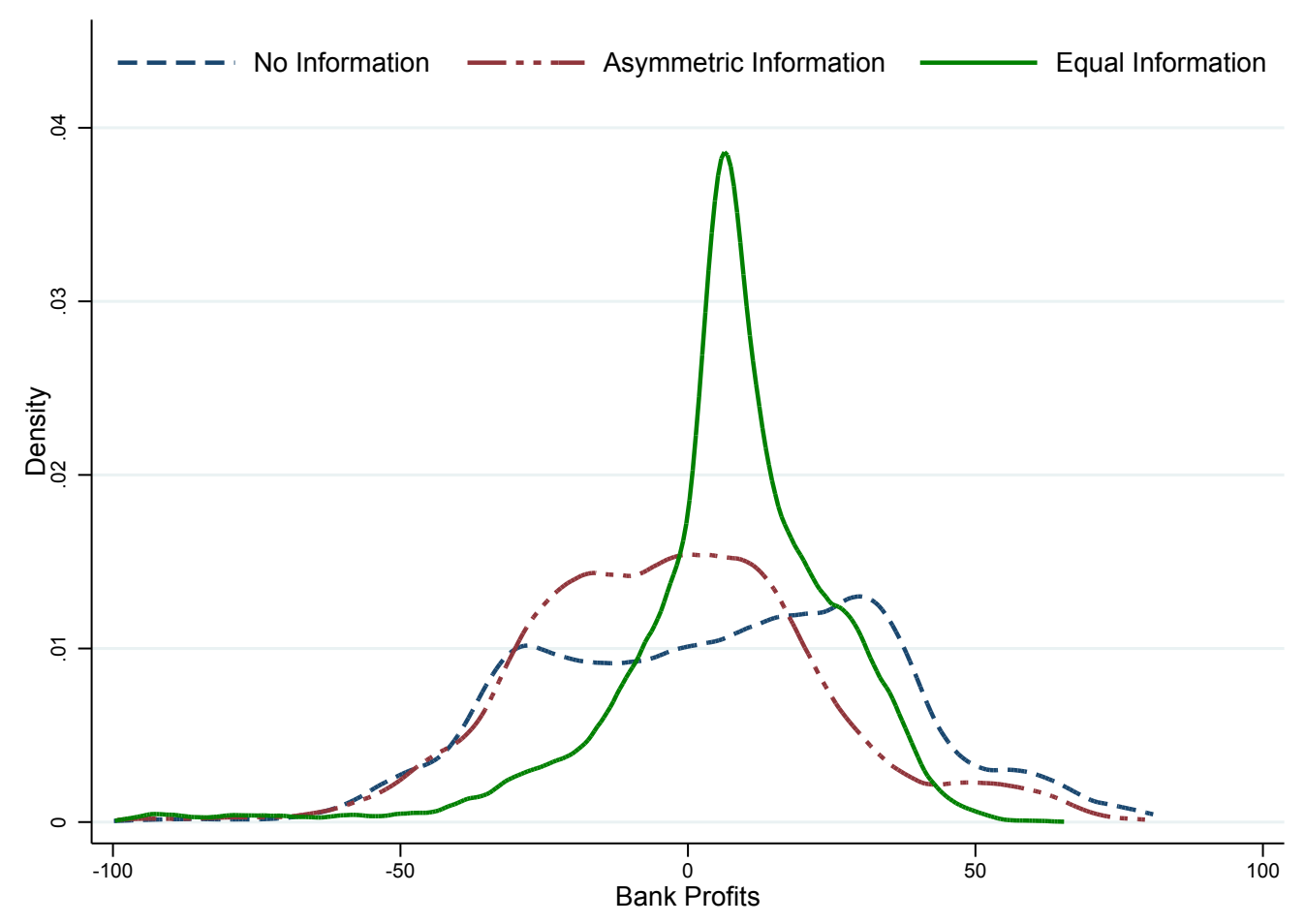

1. Under scenario: No Information, the bank and the agent have the same information at $\mathrm{t}=0$. The information set does not include $\theta$. 2. Under scenario: Asymmetric Information, the bank does not know any of the factors at $\mathrm{t}=0$, but the agent has access to $\theta_{1}$, $\theta_{2}$, and $\theta_{7}$

3. Under scenario: Equal Information, the bank and the agent have the same information at $\mathrm{t}=0$. The information set now includes $\theta_{1}$, $\theta_{2}$, and $\theta_{7}$. 


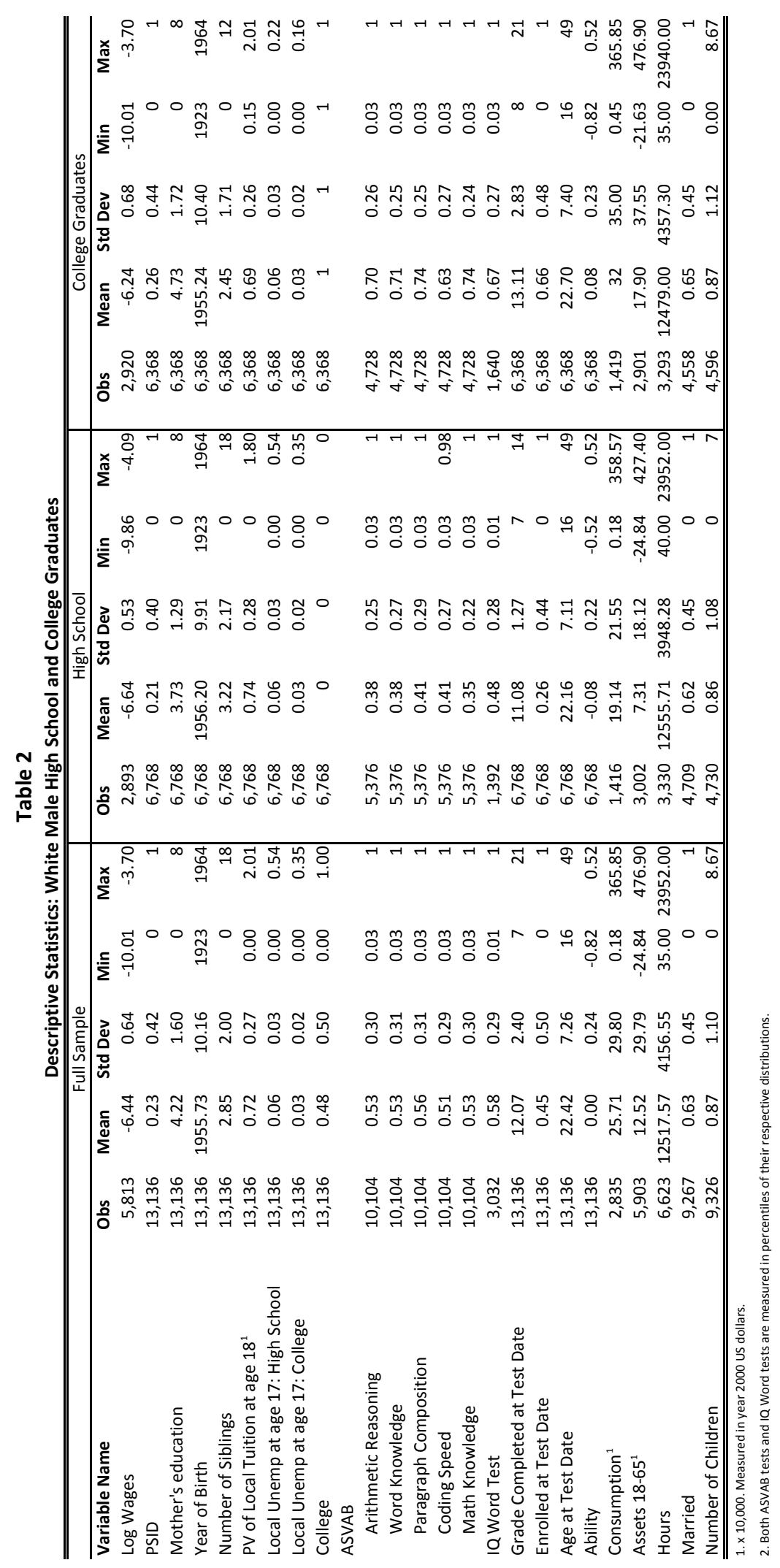




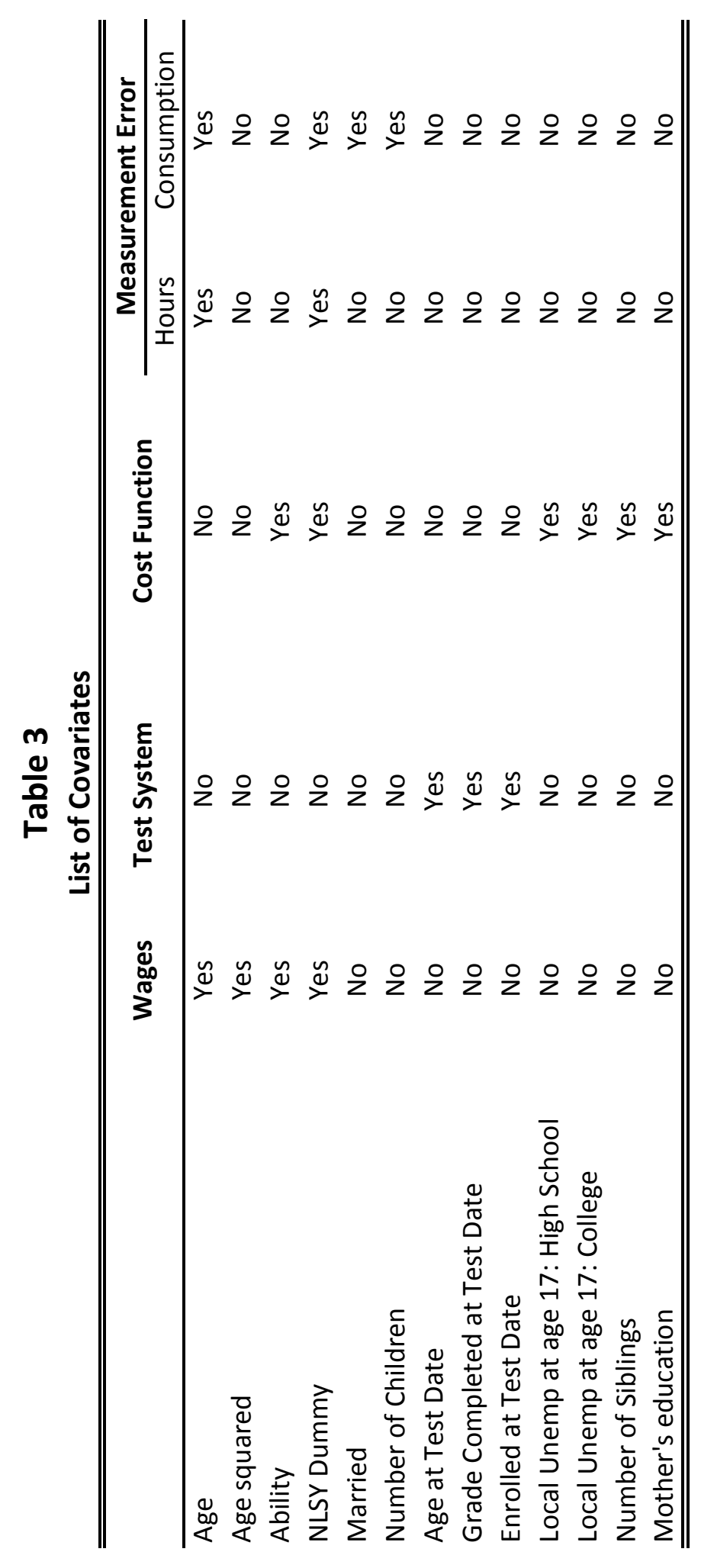


Table 4

Test for Information Set

\begin{tabular}{|c|c|c|c|c|c|c|c|}
\hline Auxiliary Parameters & $\theta_{1}$ & $\theta_{2}$ & $\theta_{3}$ & $\theta_{4}$ & $\theta_{5}$ & $\theta_{6}$ & $\theta_{7}$ \\
\hline \multirow[t]{2}{*}{ Schooling Choice } & $-0.970^{+}$ & $-0.512^{+}$ & -0.095 & -0.160 & -0.126 & 0.064 & $0.388^{+}$ \\
\hline & $(0.091)$ & $(0.144)$ & $(0.120)$ & $(0.146)$ & $(0.105)$ & $(0.180)$ & $(0.154)$ \\
\hline \multicolumn{8}{|l|}{ Log Hours } \\
\hline \multirow[t]{2}{*}{ Age 18-24 } & & $0.303^{+}$ & 0.015 & -0.007 & 0.065 & $0.269^{+}$ & $-0.128^{+}$ \\
\hline & & $(0.067)$ & $(0.038)$ & $(0.057)$ & $(0.045)$ & $(0.055)$ & $(0.062)$ \\
\hline \multirow[t]{2}{*}{ Age $25-30$} & & & 0.009 & $0.112^{+}$ & -0.027 & $0.132^{+}$ & $-0.175^{+}$ \\
\hline & & & $(0.023)$ & $(0.033)$ & $(0.027)$ & $(0.034)$ & $(0.034)$ \\
\hline \multirow[t]{2}{*}{ Age 31-36 } & & & & $0.081^{+}$ & $-0.038^{+}$ & $0.102^{+}$ & $-0.186^{+}$ \\
\hline & & & & $(0.021)$ & $(0.014)$ & $(0.017)$ & $(0.019)$ \\
\hline \multirow[t]{2}{*}{ Age $37-42$} & & & & & $-0.044^{+}$ & $0.110^{+}$ & $-0.126^{+}$ \\
\hline & & & & & $(0.014)$ & $(0.020)$ & $(0.015)$ \\
\hline \multirow[t]{2}{*}{ Age $43-48$} & & & & & & $0.140^{+}$ & $-0.101^{+}$ \\
\hline & & & & & & $(0.037)$ & $(0.031)$ \\
\hline \multirow[t]{2}{*}{ Age 49-54 } & & & & & & & $-0.265^{+}$ \\
\hline & & & & & & & $(0.103)$ \\
\hline \multicolumn{8}{|l|}{ Log Consumption } \\
\hline \multirow[t]{2}{*}{ Age $25-30$} & & & 0.117 & 0.059 & -0.096 & -0.055 & -0.103 \\
\hline & & & $(0.064)$ & $(0.097)$ & $(0.066)$ & $(0.080)$ & $(0.078)$ \\
\hline \multirow[t]{2}{*}{ Age 31-36 } & & & & $0.130^{+}$ & -0.023 & 0.003 & $-0.277^{+}$ \\
\hline & & & & $(0.061)$ & $(0.036)$ & $(0.049)$ & $(0.051)$ \\
\hline \multirow[t]{2}{*}{ Age $37-42$} & & & & & -0.045 & 0.133 & -0.048 \\
\hline & & & & & $(0.061)$ & $(0.074)$ & $(0.068)$ \\
\hline \multirow[t]{2}{*}{ Age $43-48$} & & & & & & 0.075 & -0.093 \\
\hline & & & & & & $(0.074)$ & $(0.076)$ \\
\hline \multirow[t]{2}{*}{ Age 49-54 } & & & & & & & 0.165 \\
\hline & & & & & & & $(0.183)$ \\
\hline
\end{tabular}

1. Let $\mathrm{g}(\mathrm{I})$ be the predicted choice as a function of the information set $\mathrm{I}$. The factor not included in $\mathrm{I}$, are added to the choice's contribution to the likelihood, and we test whether their associated parameters are different from zero.

2. Standard Errors in parenthesis.

3. ${ }^{+}$Significantly different from zero, at levels of $5 \%$ or less. 


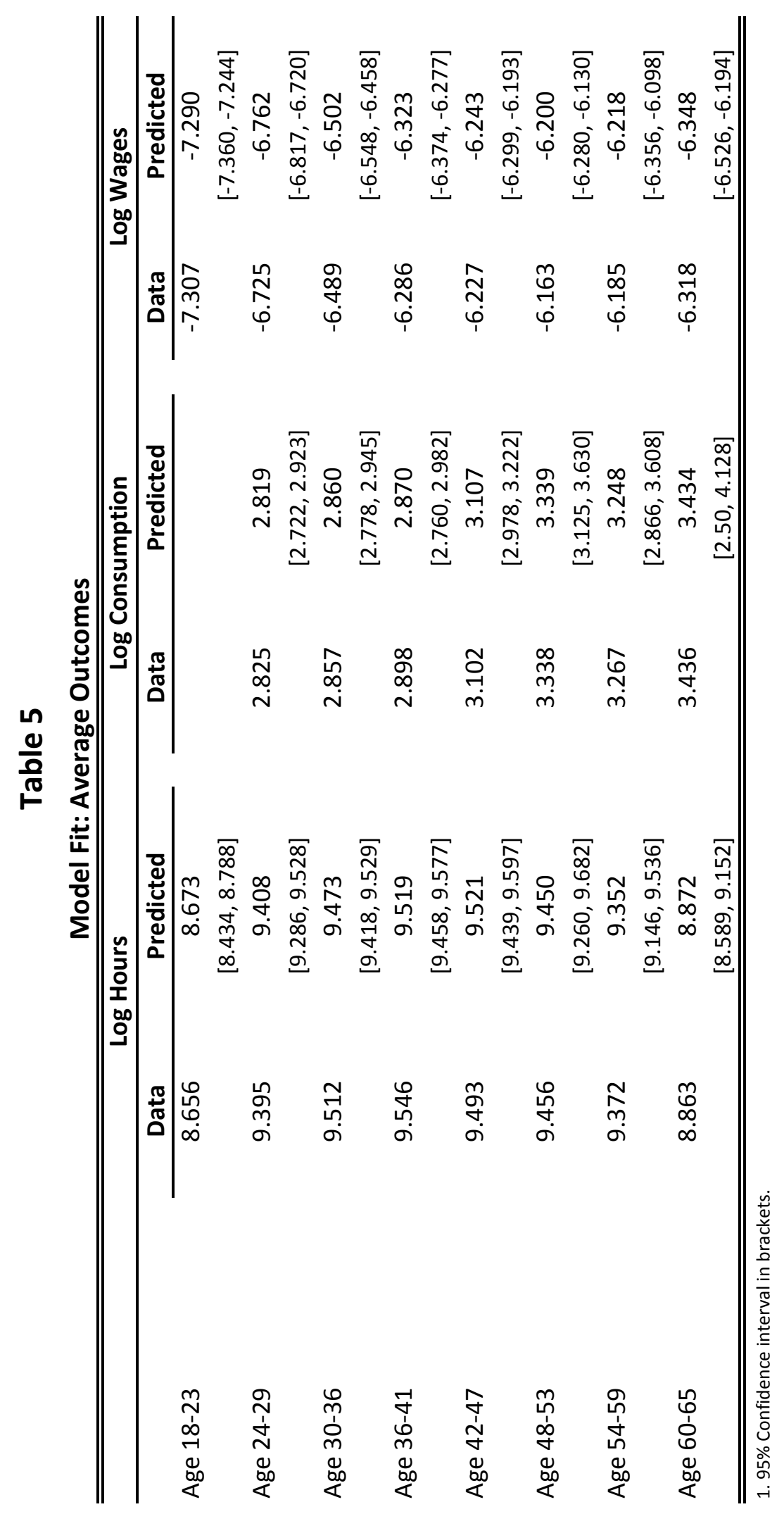




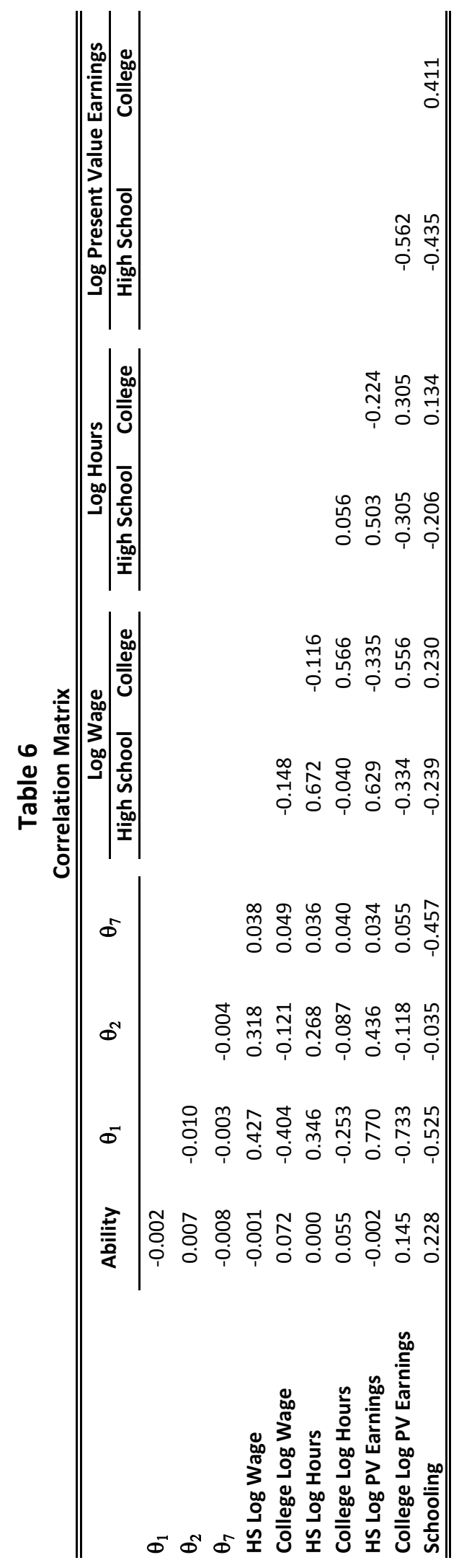




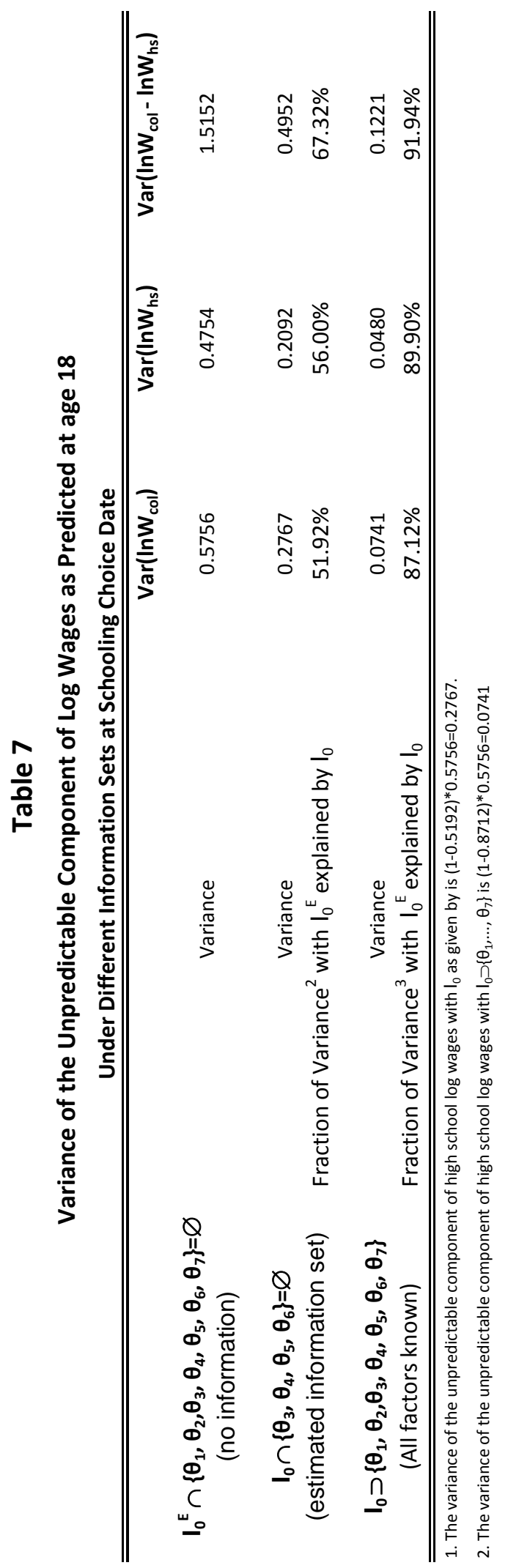




\section{Table 8}

Schooling Choice under Uncertainty and under Certainty with Borrowing Limits Unchanged

\begin{tabular}{ccc}
\hline \hline \multirow{2}{*}{ Choice Under Uncertainty } & \multicolumn{2}{c}{ Choice Under Certainty } \\
\cline { 2 - 3 } & $\begin{array}{c}\text { High School } \\
\text { Choose HS: } 46.03 \%\end{array}$ & $\begin{array}{c}\text { College } \\
\text { Choose Col: } 53.97 \%\end{array}$ \\
$\begin{array}{c}\text { High School } \\
\text { Choose HS: } 51.69 \% \\
\text { College } \\
\text { Choose Col: } 48.31 \%\end{array}$ & $78.84 \%$ & $21.16 \%$ \\
\hline \hline
\end{tabular}

1. For example, of the $51.69 \%$ of people who originally chose high school, $78.84 \%$ still do, while $21.16 \%$ now choose college. This leads to a total of $46.03 \%$ choosing high school under certainty.

\section{Table 9}

Percentage of People Who Choose College Under Different Scenarios

\begin{tabular}{ll}
\hline \hline Original Economy & $48.31 \%$ \\
Certainty with Credit Constraints & $53.97 \%$ \\
Complete markets & $59.26 \%$ \\
\hline \hline
\end{tabular}

\section{Table 10}

Schooling Choice under Uncertainty and under Complete Markets

\begin{tabular}{ccc}
\hline \hline \multirow{2}{*}{ Choice Under Uncertainty } & \multicolumn{2}{c}{ Choice Under Complete Market } \\
\cline { 2 - 3 } High School & $\begin{array}{c}\text { High School } \\
\text { Choose HS: } 40.74 \%\end{array}$ & $\begin{array}{c}\text { College } \\
\text { Choose Col: } 59.26 \%\end{array}$ \\
$\begin{array}{c}\text { Choose HS: } 51.69 \% \\
\text { College } \\
\text { Choose Col: } 48.31 \%\end{array}$ & $77.46 \%$ & $22.54 \%$ \\
\hline \hline
\end{tabular}

1. For example, of the $51.69 \%$ of people who originally chose high school, $77.46 \%$ still do, while $22.54 \%$ now choose college. This leads to a total of $40.74 \%$ choosing high school under complete market. 
Table 12

Ability and Factors for a \$12.5 Minimum Wage Insurance under Different Information Scenarios

\begin{tabular}{ccc}
\hline \hline & \multicolumn{2}{c}{ Scenario 1: No Information } \\
\hline & \multicolumn{2}{c}{ Average value for individuals who } \\
& Buy Insurance & Do not Buy Insurance \\
\cline { 2 - 3 } Ability & 0.0179 & -0.0065 \\
$\boldsymbol{\theta}_{\mathbf{1}}$ & 0.0561 & -0.0052 \\
$\boldsymbol{\theta}_{\mathbf{2}}$ & -0.0089 & -0.0049 \\
$\boldsymbol{\theta}_{\mathbf{7}}$ & -0.0191 & 0.0017 \\
$\%$ of Population & $17.90 \%$ & $82.10 \%$ \\
\hline
\end{tabular}

Scenario 2: Asymmetric Information

Average value for individuals who

\begin{tabular}{ccc}
\hline & \multicolumn{2}{c}{ Average value for individuals who } \\
& Buy Insurance & Do not Buy Insurance \\
\cline { 2 - 3 } Ability & -0.0246 & 0.0018 \\
$\boldsymbol{\theta}_{\mathbf{1}}$ & 0.3550 & -0.0894 \\
$\boldsymbol{\theta}_{\mathbf{2}}$ & -0.3267 & 0.0332 \\
$\boldsymbol{\theta}_{\mathbf{7}}$ & -0.2714 & 0.0575 \\
\% of Population & $14.92 \%$ & $85.08 \%$ \\
\hline
\end{tabular}

Scenario 3: Equal Information

Average value for individuals who

\begin{tabular}{ccc} 
& \multicolumn{2}{c}{ Average value for individuals who } \\
& Buy Insurance & Do not Buy Insurance \\
\cline { 2 - 3 } Ability & -0.0449 & 0.0045 \\
$\boldsymbol{\theta}_{\mathbf{1}}$ & -0.1962 & 0.0037 \\
$\boldsymbol{\theta}_{\mathbf{2}}$ & -0.3702 & 0.0336 \\
$\boldsymbol{\theta}_{\mathbf{7}}$ & -0.2170 & 0.0433
\end{tabular}

$\%$ of Population $\quad 13.40 \% \quad 86.60 \%$

1. Under scenario 1, the bank and the agent have the same information at $\mathrm{t}=0$. The information set does not include $\boldsymbol{\theta}$.

2. Under scenario 2 , the bank does not know any of the factors at $t=0$, but the agent has access to $\theta_{1}, \theta_{2}$, and $\theta_{7}$.

3. Under scenario 3 , the bank and the agent have the same information at $t=0$. The information set now includes $\theta_{1}, \theta_{2}$, and $\theta_{7}$. 


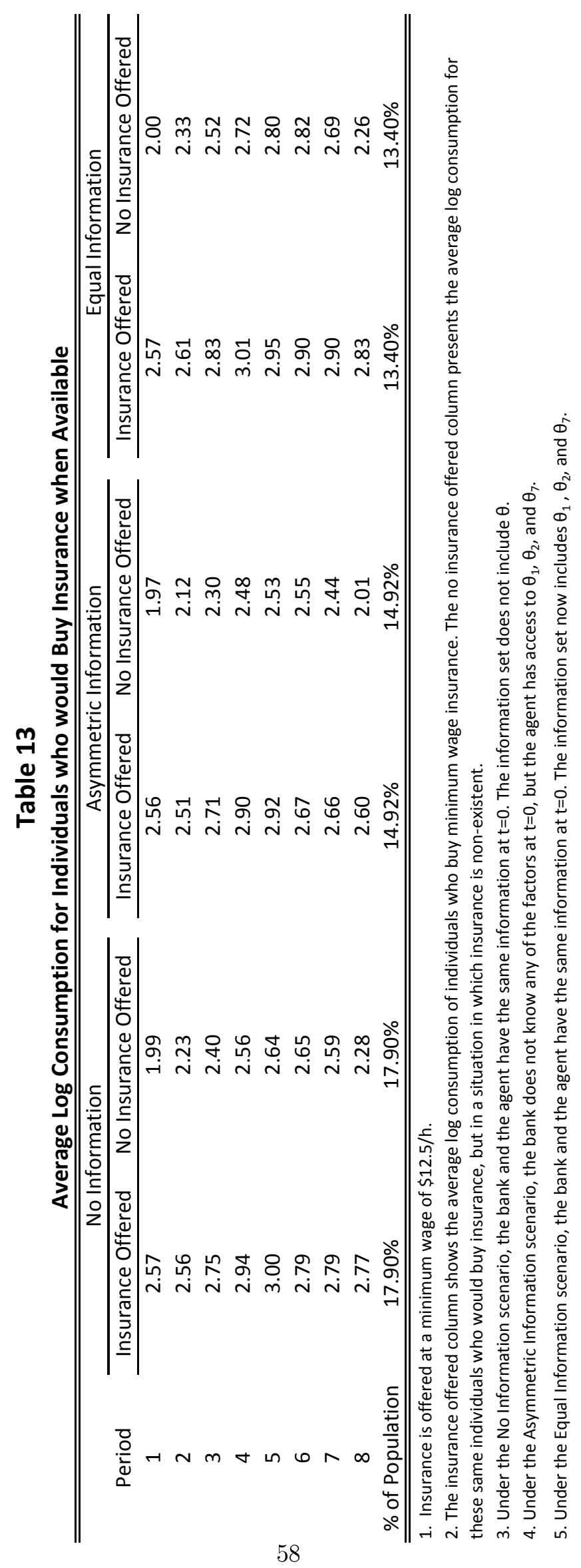




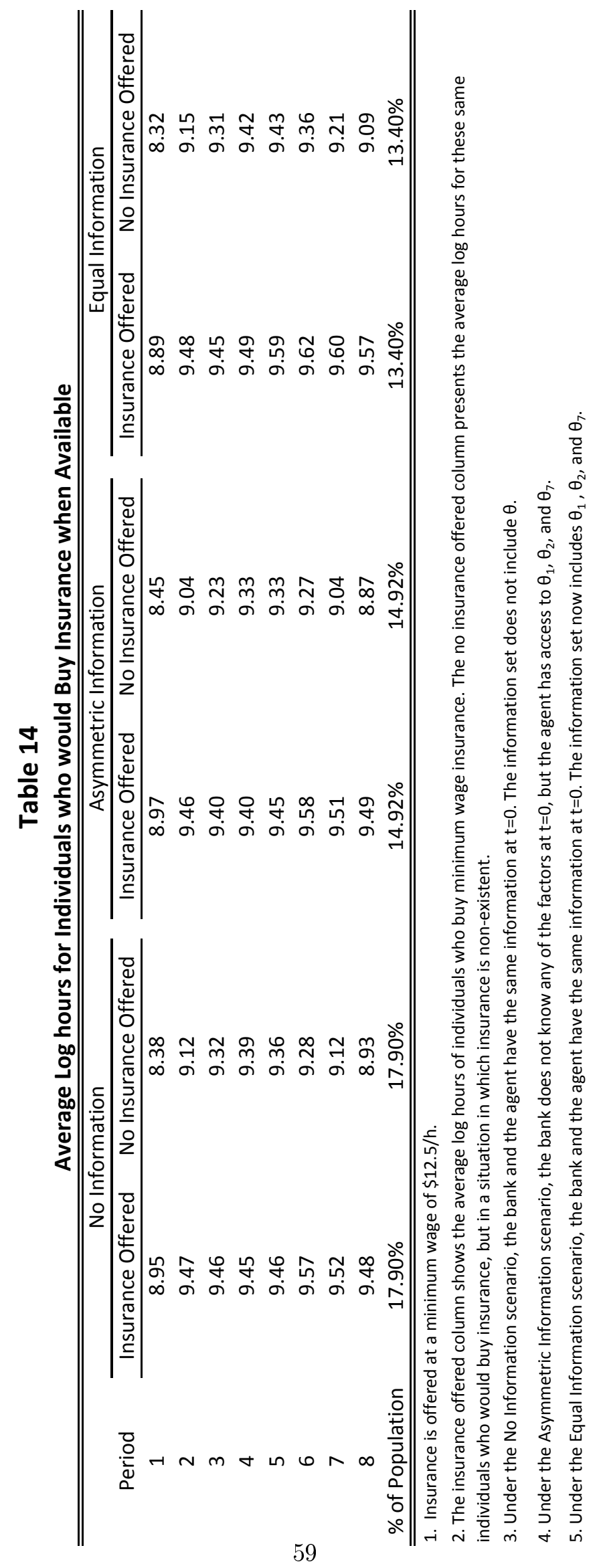




\section{Table 15}

\section{College Attendance under Different Information Scenarios for Insurance Buyers}

\begin{tabular}{lccc}
\hline \hline & Insurance Offered & No Insurance Offered & \% of Population \\
\cline { 2 - 4 } No Information & $53.41 \%$ & $58.88 \%$ & $17.90 \%$ \\
Asymmetric Information & $50.91 \%$ & $53.74 \%$ & $14.92 \%$ \\
Equal Information & $34.33 \%$ & $37.65 \%$ & $13.40 \%$ \\
\hline \hline
\end{tabular}

1. Insurance is offered at a minimum wage of $\$ 12.5 / \mathrm{h}$.

2. The insurance offered presents the education attendance decisions of individuals who buy minimum wage insurance. The no insurance offered presents the education attendance decisions for those same individuals who would buy insurance, but in a simulation in which insurance is not existent.

3. Under scenario 1: No Information, the bank and the agent have the same information at $\mathrm{t}=0$. The information set does not include $\theta$.

4. Under scenario 2: Asymmetric Information, the bank does not know any of the factors at $\mathrm{t}=0$, but the agent has access to $\theta_{1}, \theta_{2}$, and $\theta_{7}$.

5. Under scenario 3: Equal Information, the bank and the agent have the same information at $\mathrm{t}=0$. The information set now includes $\theta_{1}, \theta_{2}$, and $\theta_{7}$. 
Table 16

Schooling Choice under Different Information Scenarios

with Minimum Wage Insurance

\begin{tabular}{|c|c|c|}
\hline \multirow{3}{*}{ No Information } & \multicolumn{2}{|c|}{ Asymmetric Information } \\
\hline & High School & College \\
\hline & Choose HS: $52.19 \%$ & Choose Col: $47.81 \%$ \\
\hline \multicolumn{3}{|l|}{ High School } \\
\hline Choose HS: $49.76 \%$ & $59.98 \%$ & $40.02 \%$ \\
\hline \multicolumn{3}{|l|}{ College } \\
\hline \multirow[t]{2}{*}{ Choose Col: $50.24 \%$} & $44.47 \%$ & $55.53 \%$ \\
\hline & \multicolumn{2}{|c|}{ Equal Information } \\
\hline \multirow[t]{2}{*}{ No Information } & High School & College \\
\hline & Choose HS: $52.50 \%$ & Choose Col: $47.50 \%$ \\
\hline \multicolumn{3}{|l|}{ High School } \\
\hline Choose HS: $49.76 \%$ & $60.36 \%$ & $39.64 \%$ \\
\hline \multicolumn{3}{|l|}{ College } \\
\hline \multirow[t]{2}{*}{ Choose Col: $50.24 \%$} & $44.72 \%$ & $55.28 \%$ \\
\hline & \multicolumn{2}{|c|}{ Equal Information } \\
\hline \multirow[t]{2}{*}{ Asymmetric Information } & High School & College \\
\hline & Choose HS: $52.50 \%$ & Choose Col: $47.50 \%$ \\
\hline \multicolumn{3}{|l|}{ High School } \\
\hline Choose HS: $52.19 \%$ & $99.51 \%$ & $0.49 \%$ \\
\hline \multicolumn{3}{|l|}{ College } \\
\hline Choose Col: $47.81 \%$ & $1.18 \%$ & $98.82 \%$ \\
\hline
\end{tabular}

1. Insurance is offered at a minimum wage of $\$ 12.5 / \mathrm{h}$.

2. Under scenario 1: No Information, the bank and the agent have the same information at $t=0$. The information set does not include $\theta$.

3. Under scenario 2: Asymmetric Information, the bank does not know any of the factors at $\mathrm{t}=0$, but the agent has access to $\theta_{1}, \theta_{2}$, and $\theta_{7}$.

4. Under scenario 3: Equal Information, the bank and the agent have the same information at $\mathrm{t}=0$. The information set now includes $\theta_{1}, \theta_{2}$, and $\theta_{7}$. 


\section{Table 17}

\section{Average Welfare and Bank Profits under Different Information Scenarios}

\begin{tabular}{lccc}
\hline \hline & No Information & Asymmetric Information & Equal Information \\
Average Welfare & 32.68 & 41.79 & 41.83 \\
Bank Profits & 0.92 & -0.88 & 1.01 \\
\hline \hline
\end{tabular}

1. The welfare is average present value of consumer life-cycle utility.

2. The bank profits are average profits earned from the individuals who buy the insurance.

3. Under scenario 1: No Information, the bank and the agent have the same information at $\mathrm{t}=0$. The information set does not include $\theta$.

4. Under scenario 2: Asymmetric Information, the bank does not know any of the factors at $\mathrm{t}=0$, but the agent has access to $\theta_{1}, \theta_{2}$, and $\theta_{7}$.

5. Under scenario 3: Equal Information, the bank and the agent have the same information at $\mathrm{t}=0$. The information set now includes $\theta_{1}, \theta_{2}$, and $\theta_{7}$. 